Check for updates

Cite this: Chem. Soc. Rev., 2020, 49,554

Received 22nd August 2019

DOI: $10.1039 / c 9 c s 00588 a$

rsc.li/chem-soc-rev

\section{A review of defect structure and chemistry in ceria and its solid solutions $\dagger$}

\author{
Rafael Schmitt, (D) $\ddagger^{\mathrm{a}}$ Andreas Nenning, (ID) $\ddagger^{\mathrm{abc}}$ Olga Kraynis, (D) $\ddagger^{\mathrm{d}}$ Roman Korobko, ${ }^{\mathrm{ad}}$ \\ Anatoly I. Frenkel, (D) I Igor Lubomirsky, (D) ${ }^{d}$ Sossina M. Haile ${ }^{f}$ and \\ Jennifer L. M. Rupp (D)*abg
}

\begin{abstract}
Ceria and its solid solutions play a vital role in several industrial processes and devices. These include solar energy-to-fuel conversion, solid oxide fuel and electrolyzer cells, memristors, chemical looping combustion, automotive 3-way catalysts, catalytic surface coatings, supercapacitors and recently, electrostrictive devices. An attractive feature of ceria is the possibility of tuning defect-chemistry to increase the effectiveness of the materials in application areas. Years of study have revealed many features of the long-range, macroscopic characteristics of ceria and its derivatives. In this review we focus on an area of ceria defect chemistry which has received comparatively little attention - defect-induced local distortions and short-range associates. These features are non-periodic in nature and hence not readily detected by conventional X-ray powder diffraction. We compile the relevant literature data obtained by thermodynamic analysis, Raman spectroscopy, and X-ray absorption fine structure (XAFS) spectroscopy. Each of these techniques provides insight into material behavior without reliance on longrange periodic symmetry. From thermodynamic analyses, association of defects is inferred. From XAFS, an element-specific probe, local structure around selected atomic species is obtained, whereas from Raman spectroscopy, local symmetry breaking and vibrational changes in bonding patterns is detected. We note that, for undoped ceria and its solid solutions, the relationship between short range order and cation-oxygen-vacancy coordination remains a subject of active debate. Beyond collating the sometimes contradictory data in the literature, we strengthen this review by reporting new spectroscopy results and analysis. We contribute to this debate by introducing additional data and analysis, with the expectation that increasing our fundamental understanding of this relationship will lead to an ability to predict and tailor the defect-chemistry of ceria-based materials for practical applications.
\end{abstract}

\section{Basic properties and applications of ceria}

Over the past few decades, the crystal structure, electronic and ionic conductivities, elastic moduli and electromechanical

\footnotetext{
${ }^{a}$ Electrochemical Materials, Department of Materials, ETH Zurich, Switzerland

${ }^{b}$ Electrochemical Materials, Department of Materials Science and Engineering, Massachusetts Institute of Technology, Cambridge, MA, 02139, USA. E-mail: jrupp@mit.edu

${ }^{c}$ TU Wien, Institute of Chemical Technologies and Analytics, Vienna, 1060, Austria

${ }^{d}$ Department Materials and Interfaces, Weizmann Institute of Science, Rehovot 76100, Israel

${ }^{e}$ Department of Materials Science and Chemical Engineering, Stony Brook University, Stony Brook, NY 11794, USA

${ }^{f}$ Materials Science and Engineering, Northwestern University, Evanston, IL, USA ${ }^{g}$ Electrochemical Materials, Department of Electrical Engineering \& Computer Science, Massachusetts Institute of Technology, Cambridge, MA, 02139, USA $\dagger$ Electronic supplementary information (ESI) available. See DOI: 10.1039/c9cs00588a \# Shared first-co-authorship, these authors have equally contributed to this manuscript.
}

behavior of ceria, $\mathrm{CeO}_{2}$, and its doped derivatives, have been studied and the materials have been found to be suited to a variety of important industrial applications. ${ }^{1}$ Beyond the functional properties, these oxides are generally inert against reactions and hence are compatible with many materials, both biological and inorganic, they are stable in harsh environments, including temperatures up to at least $2000{ }^{\circ} \mathrm{C}$, and cerium, despite being a lanthanide, is rather abundant in the earth's crust, comparable in abundance to copper. ${ }^{2}$ These features add to the suitability of this class of materials to a wide range of devices.

The properties of ceria are closely linked to its rather stable cubic fluorite $(F m \overline{3} m)$ crystal structure, Fig. 1a, which has four $\mathrm{CeO}_{2}$ formula units per cubic unit cell. The Ce atoms are arranged in a face centered cubic array, occupying the 4a sites of the $F m \overline{3} m$ space group, and the oxygen anions fill the eight tetrahedral $8 \mathrm{c}$ sites. The arrangement gives 8-fold coordination of $\mathrm{Ce}$ and 4 -fold tetrahedral coordination of $\mathrm{O}$ ions. The properties of the material can be readily manipulated within 
the framework of this robust crystal structure by extrinsic doping to create solid solutions. In particular, cations of similar size to $\mathrm{Ce}^{4+}$ but with different valence are compensated by the creation of functional point defects. When the dopants are trivalent acceptor cations, henceforth Do, (Fig. 1b), the missing positive charge is compensated by the formation of one extrinsic oxygen vacancy per two dopant elements (Fig. 1c), and the stoichiometry is $\mathrm{Ce}_{1-x} \mathrm{Do}_{x} \mathrm{O}_{2-x / 2}$. Such acceptor doping has been widely exploited for fuel cell development, ${ }^{3,4}$ because the resulting materials, particularly in the case of Sm and Gd doping, display exceptionally high ionic conductivity. ${ }^{5}$ Divalent dopants (e.g. $\mathrm{Ca}, \mathrm{Mg}$. Ba), though generating a higher concentration of vacancies per dissolved dopant ion, result in uniformly lower conductivities than Sm and Gd dopants ${ }^{6}$ and therefore have been of much lower technological interest. The case of high valence donor dopants $\left(e . g . \mathrm{Nb}^{5+}, \mathrm{Ta}^{5+}\right)$ has been studied to an even lesser extent, in large part because the solubilities of these species is relatively low (generally on the order of 1 at\%), implying limited impact on bulk properties. ${ }^{7-9}$

Even in the absence of extrinsic dopants, a substantial concentration of point defects can exist in ceria as a result of partial reduction of $\mathrm{Ce}^{4+}$ to $\mathrm{Ce}^{3+}$. In such case, the material has the formula $\mathrm{CeO}_{2-\delta}$, where $\delta$ is the oxygen non-stoichiometry, and both ionic and electronic conductivity become significant. The reduction is generally favored at high temperatures, low gas-phase oxygen chemical potentials (i.e., low oxygen partial pressures), and on surfaces. ${ }^{10,11}$ The reversibility and ease of the reduction is implicated in the high catalytic activity of ceria for a variety of redox reactions which are reviewed e.g. by Trovarelli $^{12}$ or Montini. ${ }^{13}$ The most prominent technological application in this context is in automotive three-waycatalysts. $^{1,14}$ In order to simultaneously remove soot, carbon

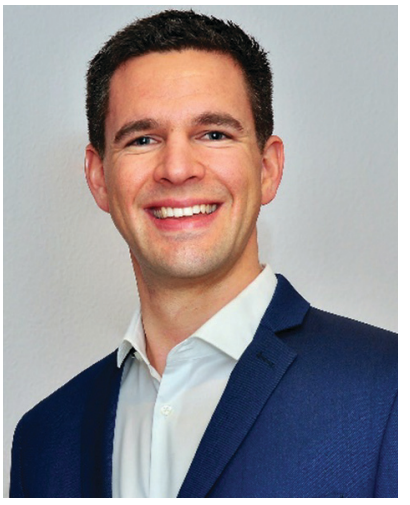

Rafael Schmitt
Rafael Schmitt is currently working on the industrialization of additive manufacturing with a focus on ceramic and metallic materials at Exentis Group $A G$ in Stetten, Switzerland. He earned his PhD degree at ETH Zurich in 2017 in the group of electrochemical materials of Prof. Jennifer L. M. Rupp. He worked on understanding the mass and charge transport in oxide-based resistive switching materials under high electric fields in view of providing design guidelines for memristive devices. Awards include the Excellence Scholarship \& Opportunity Programme for the Master's degree at ETH Zurich and the E-MRS Young Scientist Award at the E-MRS Spring Meeting in Lille, France, 2016.

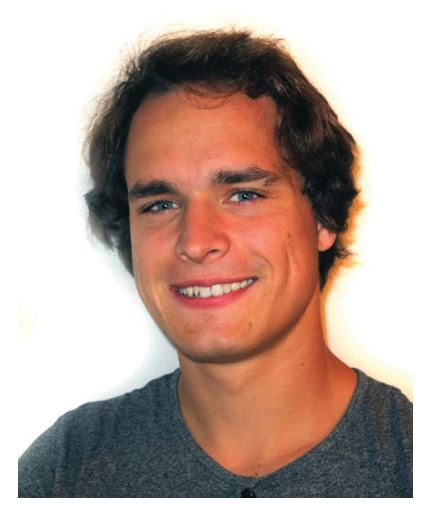

Andreas Nenning
Andreas Nenning is currently a postdoctoral researcher at $T U$ Wien, investigating the electrochemical properties and redox kinetics of acceptor doped ceria for use in solid oxide fuel cells. In 2016 and 2017, he worked in the electrochemical materials group of Prof. Jennifer Rupp, and later with her at MIT. There his work focused on the observation of ionic and electronic conduction in Gd-doped ceria as a model system for electrochemical valence change resistive memories. Another research topic was optimization of electrochemical carbon dioxide sensors. He received his $P h D$ degree in 2016 at the TU Vienna, working in Prof. Jürgen Fleig's group.

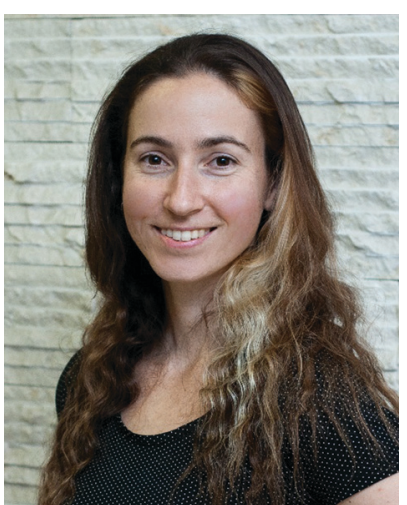

Olga Kraynis
Olga Kraynis is currently a postdoctoral researcher at the Department of Materials and Interfaces at the Weizmann Institute of Science (WIS) Israel, in the group of Prof. Igor Lubomirsky. She received a BSc in chemistry from Tel Aviv University (Israel) in 2012. She then completed her PhD in the group of Prof. Igor Lubomirsky at the WIS in 2019. The topic of her doctoral thesis was structural characterization of point defects, and their effect on mechanic and electro-mechanic properties of ceria thin film materials. Olga Kraynis is the recipient of the 2019 Professor Israel Dostrovsky Memorial Prize of Excellence.

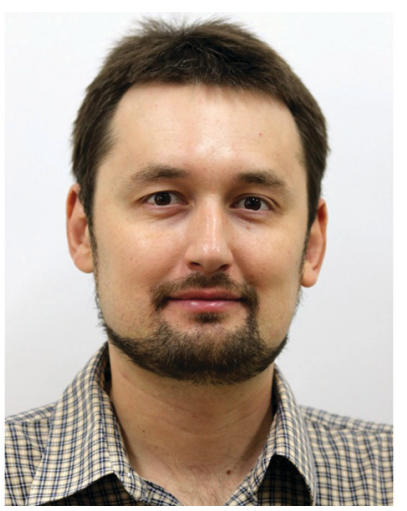

Roman Korobko
Roman Korobko is a staff scientist at the Solid-State Structural Dynamics group in the Weizmann Institute of Science. His research interests include the elastic, electronic and memristive properties of dielectrics. Roman received his $B A$ in Chemistry and BSc in Materials Engineering in Technion in 2003. He continued his studies in the Faculty of Chemistry of the WIS where he earned MSc in 2009 and $P h D$ on controlling the elastic properties of ceramics with an external electric field in 2014. He

then carried out postdoctoral research at the Electrochemical Materials group in ETH Zurich in the field of memristive materials during 2014-2017. 
monoxide, and $\mathrm{NO}_{x}$ from the exhaust stream, precise control of the oxygen-to-fuel ratio is necessary. Ceria has the capacity to serve as an oxygen buffer by actively varying its oxygen nonstoichiometry, supplying oxygen when required for carbon oxidation, and removing it as necessary for $\mathrm{NO}_{x}$ reduction. Another promising use of the oxygen storage capacity of ceria is in solar-driven thermochemical water splitting. ${ }^{15,16}$ During this cyclic process, ceria releases oxygen at extremely high temperatures (typ. $1500{ }^{\circ} \mathrm{C}$ ), which can be achieved by solar concentration. Subsequently, the material is cooled to $800-1000{ }^{\circ} \mathrm{C}$, and the oxygen vacancies generated from the prior reduction step are filled with oxygen through a water and $\mathrm{CO}_{2}$ splitting

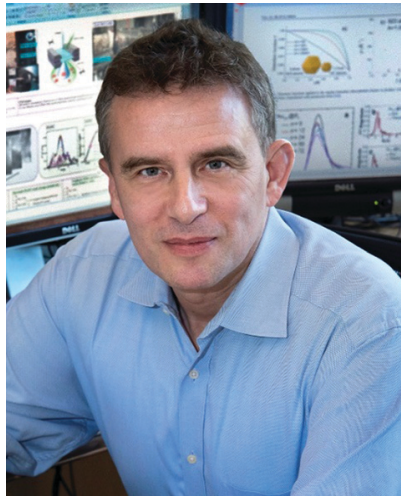

Anatoly I. Frenkel

Anatoly Frenkel is a Professor in the Department of Materials Science and Chemical Engineering at Stony Brook University and a Senior Chemist at the Division of Chemistry, Brookhaven National Laboratory. He was a Professor and Chair, Physics Department at Yeshiva University, and a Research Scientist at the University of Illinois at Urbana-Champaign. He received MSc degree from St. Petersburg University and $\mathrm{PhD}$ degree from Tel Aviv University, all in Physics. His research interests focus on development and application of synchrotron methods for structural analysis and design of nanomaterials. He is a Fellow of the American Physical Society.

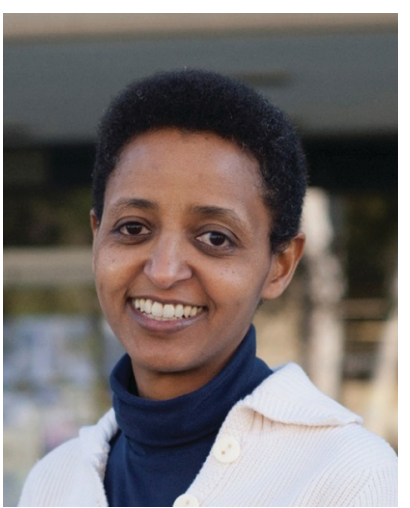

Sossina M. Haile
Sossina Haile is the Walter $P$. Murphy Professor of Materials Science and Engineering at Northwestern University. She earned her PhD in Materials Science and Engineering from the Massachusetts Institute of Technology in 1992. Haile's research broadly encompasses materials for sustainable electrochemical energy technologies. Amongst her many awards, in 2008 Haile received an American Competitiveness and Innovation Fellowship from the U.S. National Science Foundation. She is a fellow of the Materials Research Society, the American Ceramics Society, the African Academy of Sciences, and the Ethiopian Academy of Sciences, and serves on the editorial boards of Materials Horizons and Annual Review of Materials Research. reaction. Though not treated in this review, it is noteworthy that doping with tetravalent cations, $\mathrm{Zr}^{4+}$ or $\mathrm{Hf}^{4+}$, increases the ease by which the reduction of the host material occurs. ${ }^{17,18}$ This behavior is used to increase the oxygen storage capacity in exhaust catalysts, or to decrease the operating temperature of solar- and thermochemical water splitting. ${ }^{19,20}$ The partial reduction of ceria in the presence of hydrogen or other fuels furthermore provides advantages for its use as a constituent in composite anodes of solid oxide fuel cells. ${ }^{21-24}$

While the use of ceria and its doped derivatives in energy and environmental technologies as described above is relatively well-known, the reducibility and mixed conductivity of ceria are

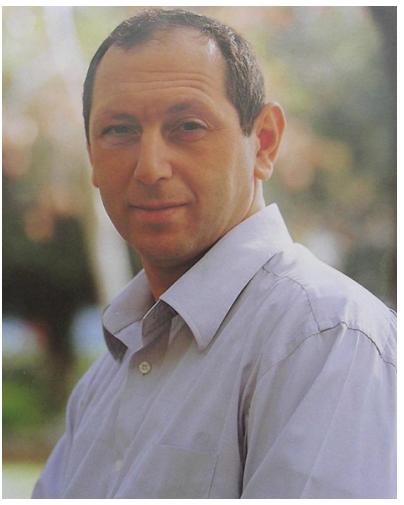

Igor Lubomirsky
Igor Lubomirsky is a professor at the Department of Materials and Interfaces at the Weizmann Institute of Science, Israel. He studied chemical engineering in Kharkov (now Ukraine) and then earned his PhD in the WIS. After postdoctoral terms in UCLA and Max Planck Institute for Solid State Research, he rejoined the WIS as a Chemistry faculty member. His studies focus on solids, in which local symmetry reduction strongly affects mechanical, electromechanical and pyroelectric properties. During the last decade, he initiated studies of anelasticity in solids with a high concentration of point defects. These solids withstand unusually large range of deformations, within which they exhibit rubber-like properties.

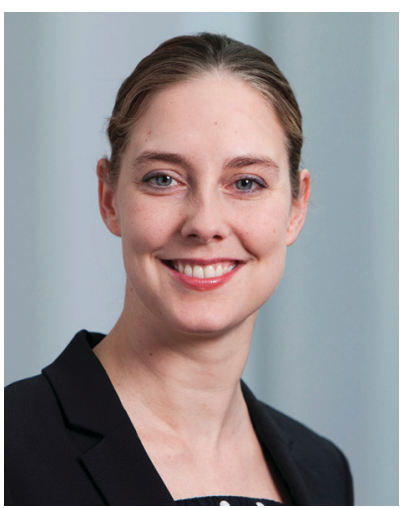

Jennifer L. M. Rupp
Jennifer Rupp is the Thomas Lord Associate Professor of Electrochemical Materials at the Department of Materials Science and Engineering and in the Department of Electrical Engineering and Computer Science at MIT. Prior she was a non-tenure track assistant professor at ETH. Her research is in designing "ceramic" materials and their properties for device functions in energy and computation. Rupp and team received several honors such as the Displaying Future Award by the company Merck 2018 for a glucose converting fuel cell chip, BASF and Volkswagen Science Award 2017 for battery research, "Top 40 international scientist under the age of 40" by World Economic Forum 2015 and others. Currently, she is associate editor at the Journal of Materials Chemistry $A$ and board member at Advanced Functional Materials and Advanced Interface Materials. 


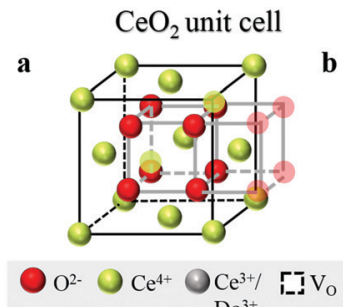

b
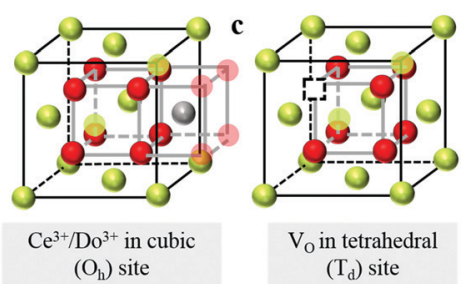

Fig. 1 Schematic of crystal structure and defects for ceria and solid solutions: (a) displays a $F m \overline{3} m$ fluorite ceria unit cell, containing 8 oxygen atoms (red) forming a simple cube embedded inside a face centered cubic (FCC) array of Ce cations (lime). Every second oxygen cube contains a $\mathrm{Ce}$ cation, as illustrated by the 4 semi-transparent oxygen atoms on the right, belonging to the next unit cell. Over all, the fluorite unit cell has 8 oxygen atoms and 4 cerium atoms. (b and c) Illustrate the two main point-defects which may or may not be preferentially nearest neighbors, depending on the dopant type and prevalence of oxygen deficiency. Cation defects, $\mathrm{Ce}^{3+}$ (in oxygen-deficient ceria) and $\mathrm{Do}^{3+}$ (in doped ceria), sit in a $\mathrm{Ce}^{4+}$ cubic site of $\mathrm{O}_{\mathrm{h}}$ symmetry with 8 -fold oxygen coordination. Every two cation defects in the structure are balanced by one oxygen-vacancy defect, $V_{O}$, which sits in a tetrahedral site of $T_{d}$ symmetry, with 4 -fold cation coordination.

also relevant to its applicability in emerging areas such as resistive switching devices ${ }^{25-28}$ for computation and data storage, and supercapacitors. $^{29}$ In the medical sector, where ceria has been found to be biocompatible with human tissue, the surface catalytic redox activity has been investigated for potential use in cancer therapy and inhibition of radiation-induced damage. ${ }^{30,31}$

Because of the extensive range of demonstrated and possible applications of trivalent (rare-earth) doped ceria, the literature is rich with studies connecting the macroscopic properties, particularly transport properties, to the material chemistry ${ }^{32-34}$ and to the long-range, averaged crystal structure, where the latter is generally determined by conventional X-ray powder diffraction. ${ }^{35,36}$ In this review we focus on an area of ceria defect chemistry that has received comparatively little attention: defect-induced local distortions and short-range associates. The selection of this topic is motivated by the recognition that several questions regarding the chemo-mechanical and electrostrictive properties of doped ceria remain open. ${ }^{37-39}$ For example, 10 mol\% Gd-doped ceria supported thin films ${ }^{37,38}$ can develop large electrostrictive stress (up to $500 \mathrm{MPa}$ ) and the electrostrictive strain coefficient is surprisingly large at low electric field frequencies. As characterized by Newnham et al. for a large variety of materials, ${ }^{40}$ the electrostrictive strain coefficient should scale with the ratio of the material elastic compliance to the dielectric permittivity. According to this scaling law, materials with large bulk modulus and dielectric constant 30-55 such as trivalent doped ceria should not produce a large electrostrictive response. ${ }^{41}$ For example, Y-stabilized $\mathrm{ZrO}_{2}$ has a strain electrostriction coefficient of the order of $10^{-21}\left(\mathrm{~m}^{2} \mathrm{~V}^{-2}\right)$. However, electrostriction coefficients of $\approx 10^{-18}-10^{-16}\left(\mathrm{~m}^{2} \mathrm{~V}^{-2}\right)$, comparable to commercial relaxor materials, have been recorded for substrate supported doped ceria films in a "cantilever" configuration, ${ }^{37,39,42}$ self-supported membranes, ${ }^{43}$ as well as for bulk ceramics. ${ }^{44}$ Moreover, Gd-doped ceria has been shown to be anelastic, meaning that the elastic moduli depend on the rate of deformation. ${ }^{45}$ These unusual properties, which emerge at trivalent dopant concentrations of $\leq 20 \mathrm{~mol} \%$, are proposed to originate from anisotropic lattice distortions in the near neighbor shells of the cations and oxygen vacancies in an average fluorite structure. ${ }^{38,46}$

This review draws from the extensive literature derived from thermogravimetric measurements, ${ }^{18,47-51}$ from which defect association in partially reduced ceria compositions can be inferred, and from local structural measurements, specifically X-ray absorption fine structure (XAFS) spectroscopy and Raman spectroscopy. These spectroscopic methods are exquisitely suited to the detection of non-periodic distortions and can assist in the search for suitable descriptors of the interaction of oxygen anions and vacancies with dopant or $\mathrm{Ce}^{3+}$ ions, e.g. defect associates and lattice distortions. Additional suitable methods for addressing short-range interatomic distances such as neutron and X-ray total scattering analysis using Pair Distribution Function (PDF) were recently reviewed by Coduri et al. in ref. 34 . The conclusions from these data will be briefly discussed alongside XAFS results. Similarly, nuclear magnetic resonance (NMR) spectroscopy can give insights into local structural configurations, and conclusions in this area are discussed alongside the Raman spectroscopy results. Other methods like oxygen isotope exchange and depth profiling (IEDP) by secondary ion mass spectroscopy (SIMS) yield valuable complementary information on oxygen exchange and diffusion kinetics, but do not shed light on the local structure considered in this review. ${ }^{52-55}$

The thermogravimetric measurements, analyzed in conjunction with appropriate defect models, reveal that, as soon as defect concentration become non-negligible, the thermodynamics of reduction of undoped and (to a lesser extent) doped ceria deviate from simple solution behavior. In such case, rather than isolated, non-interacting defects, associates in the form of neutral trimers dominate the defect chemistry. ${ }^{56,57}$ Raman spectroscopy reveals the emergence of new peaks when defects are introduced into ceria, providing a sensitive tool for investigating local structure. Extended XAFS (EXAFS) spectroscopy has been used to measure the distance from Ce or dopant cations to their nearest neighbors (oxygen anions) or next nearest neighbors (other cations). The average nearestneighbor distances of doped ceria show that the lattice strongly deviates from fluorite symmetry at the unit cell level. ${ }^{58,59}$ This stands in a seeming contradiction with the XRD studies which average over all unit cells and indicate fluorite symmetry for acceptor doping levels up to $20 \mathrm{~mol} \% .^{35,60-62}$ It is evident that the global average does not provide the full structural description and a multimodal approach, combining the XRD and EXAFS studies to provide a picture of actual structure, is needed. In agreement with the EXAFS findings, Raman spectra of extrinsically doped and reduced undoped ceria, demonstrate additional defectrelated Raman modes, indicating that the fluorite symmetry is no longer present on the unit cell level.

Combining the results across methods that are capable of directly probing local cation-oxygen, and oxygen-vacancy related lattice interactions and distortions enables identification of 
new parameters affecting electro/thermo-chemo-mechanics of ceria solid solutions. In turn, the insights afforded from this framework can reveal the role of defect clustering and local structural distortions on reducibility and the generation of surprisingly large electrostriction. Such factors must be considered when designing ceria based materials for a wide range of applications.

\section{Thermodynamic studies of the defect-chemistry of ceria, without and with dopants}

We review in this section the state of understanding of the defect-chemistry of ceria as developed largely using thermogravimetric methods and conductivity measurements in combination with defect chemical modeling and more recent atomistic simulations. With such techniques, information on the enthalpies and entropies of defect interactions, as well as candidates for defect clusters are accessible. As we show later in this review, Raman and XAFS spectroscopies can directly reveal the local chemical environment of defects. These techniques provide even deeper insights into the nature, concentration and degree of association of such defects as relevant for fully elucidating the structure-property relationships.

Ceria is a wide bandgap semiconductor with an experimentally measured bandgap of $\sim 6 \mathrm{eV}$ from the valence band with predominantly oxygen $2 \mathrm{p}$ character to the conduction band with predominantly cerium $5 \mathrm{~d}$ character. ${ }^{63}$ Localized, empty $4 \mathrm{f}$ states lie within the gap, ${ }^{64}$ leading some to describe the bandgap to be $\sim 3 \mathrm{eV},{ }^{65}$ which is, however, the distance from the valence band to the bottom of the empty $4 \mathrm{f}$ band. As already noted, doping with tri- or divalent cations introduces extrinsic oxygen vacancies, which balance the effective negative charge of the dopant species. Exposure to high temperature or reducing gas conditions causes a loss of oxygen to the gas phase, generating intrinsic oxygen vacancies. These vacancies are charge-balanced by electronic defects in form of $\mathrm{Ce}^{3+}$ ions and, depending on specific conditions, the concentration on intrinsic defects can dominate bulk properties. ${ }^{50}$ The reactions and mass action laws characterizing the redox behavior are summarized in Table 1 (eqn (1)-(10)), where the defect species are described using Kroger-Vink notation. ${ }^{66}$ The electronic defect, $\mathrm{Ce}_{\mathrm{Ce}}^{\prime}$, expressed in these equations describes a cerium ion that has trapped an electron in its vicinity. ${ }^{67,68}$ The trapping gives rise to a small polaron, i.e., a quasiparticle which appears when an electron interacts with the polarization of its surrounding ions. ${ }^{69}$ Electronic structure calculations suggest that the polarons fill states that are created as a result of the reduction and lie just below the unoccupied $4 \mathrm{f}$ states at a distance of 2 to $2.4 \mathrm{eV}$ from the valence band, as sketched in Fig. 2. ${ }^{64,70,71}$ Their position is also $\sim 1 \mathrm{eV}$ below the Fermi level, as experimentally observed in ambient pressure XPS (X-ray photoelectron spectroscopy) measurements on reduced ceria. $^{10,72}$

Under conditions in which the non-stoichiometry $\delta$ is sufficiently small that defects are non-interacting, the reduction of ceria is described by eqn (1) with the corresponding mass

Table 1 Defect chemical reactions describing the redox behavior of undoped, $\mathrm{CeO}_{2-\delta}$, and doped, $\mathrm{Ce}_{1-x} \mathrm{Do}_{x} \mathrm{O}_{2-x / 2-\delta}$, ceria, where $\mathrm{Do}{ }^{3+}$ is a trivalent dopant species

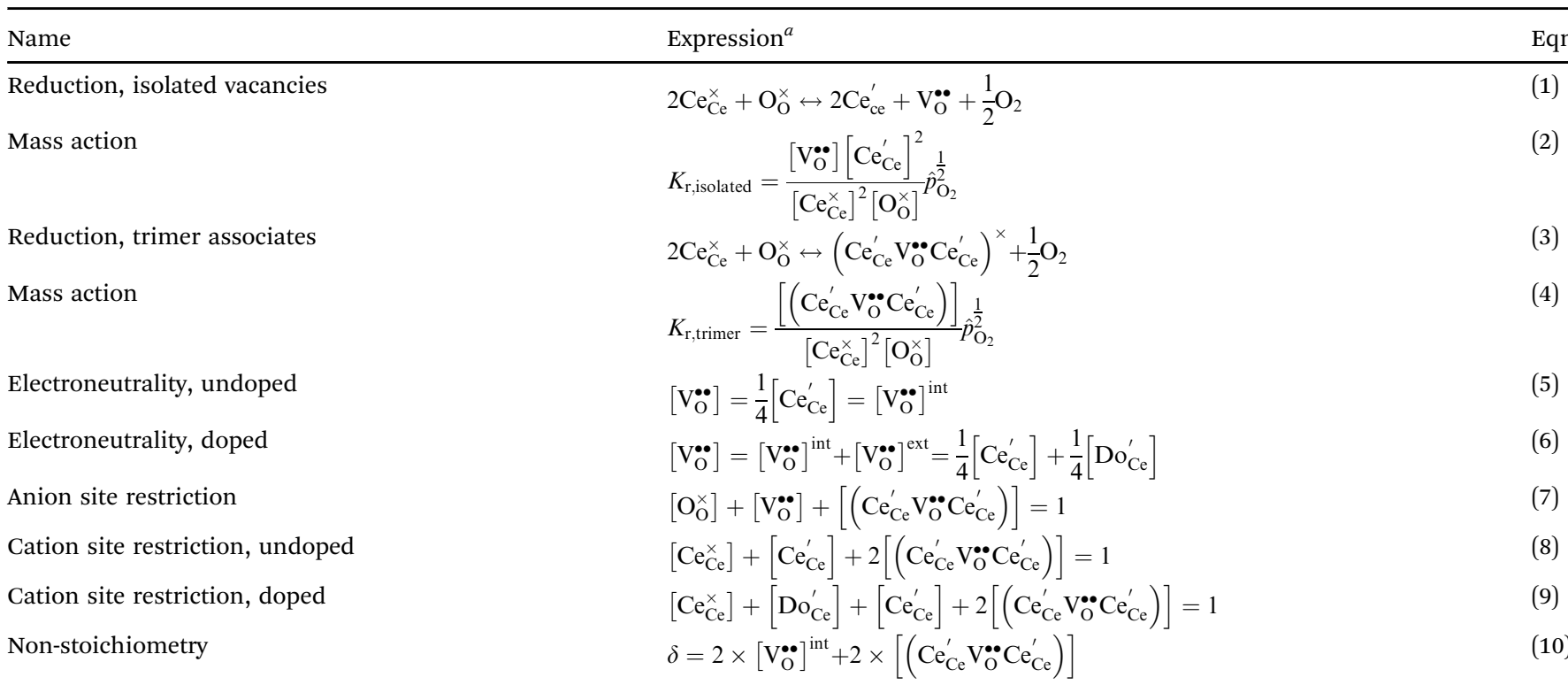

${ }^{a}$ Square brackets indicate the fractional (unitless) site occupancies; there are two oxygen and one cation sites per formula unit, electroneutrality equations differ by a factor of 2 when expressed in volumetric concentrations. $\left[\mathrm{V}_{\mathrm{O}}^{\bullet \bullet}\right]$, including intrinsic (int) and extrinsic (ext) contributions, and $\left[\mathrm{Ce}_{\mathrm{Ce}}^{\prime}\right]$ refer to the concentration of vacancies and electronic defects not in associates. $\hat{p}_{\mathrm{O}_{2}}$ is the oxygen pressure relative to the standard oxygen pressure of $1 \mathrm{~atm}$. 


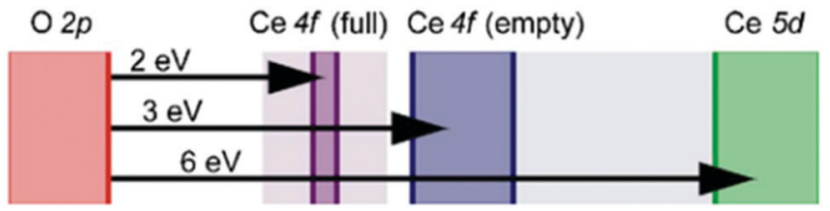

Fig. 2 Schematic band diagram of ceria containing electronic defects. The $\mathrm{O}_{2 p}$ states form the valence band and the $\mathrm{Ce}_{5 d}$ states the conduction band. The $\mathrm{Ce}_{4 \mathrm{f}}$ states labelled 'full' are absent when the material has no electronic defects. Shading is used to indicate uncertainty in the band positions. Reproduced from ref. 64 with permission from AIP Publishing, copyright 2007.

action law, eqn (2). ${ }^{51,68}$ When the site occupancies of $\mathrm{Ce}_{\mathrm{Ce}}^{\times}$and $\mathrm{O}_{\mathrm{O}}^{\times}$ are close to 1 , the equilibrium constant can be approximated as

$$
K_{\mathrm{r}, \text { isolated }} \approx\left[\mathrm{V}_{\mathrm{O}}^{\bullet \bullet}\right]\left[\mathrm{Ce}_{\mathrm{Ce}}^{\prime}\right]^{2} \hat{p}_{\mathrm{O}_{2}}^{\frac{1}{2}}
$$

Together with the electroneutrality condition expressed in eqn (5), the above implies vacancy and electronic defect concentrations that vary with oxygen partial pressure according to a power law exponent of $-\frac{1}{6}$. Experimentally, power law exponents close to this value have been observed in oxygen partial pressure dependent conductivity measurements. ${ }^{68}$ Such measurements express the thermodynamics because they reflect the variation in the polaron defect (i.e. carrier) concentration. In contrast to transport measurements, thermogravimetric measurements tend to show slightly larger exponents. ${ }^{50,51}$ This is undoubtedly due to the difficulty in measuring mass changes in the regimes where $\delta$ is small, whereas accurate conductivity measurements are readily performed under such conditions.

At high oxygen non-stoichiometry, encountered at low oxygen partial pressure and high temperature, defect interactions become important. The mass action law, eqn (2), in principle, assumes dilute solution behavior and taken alone, is incompatible with such interactions. The problem can be treated by replacing the concentration terms in the mass action equation with activities, or by introducing additional reactions to describe specific types of defect interactions such as associates, as given, for example, in eqn (3). The latter approach has the benefit of revealing the underlying defect-chemistry.

The early literature suggested that the relevant defect interactions in highly reduced ceria were between free electrons and oxygen vacancies, generating singly ionized $\mathrm{V}_{\mathrm{O}}^{\bullet}$ or neutral species $\left(\mathrm{V}_{\mathrm{O}}^{\times}\right),{ }^{68}$ with the singly ionized defect dominating the behavior. More recent analysis indicates that, instead, associates are formed between $\mathrm{Ce}_{\mathrm{Ce}}^{\prime}$ ions and oxygen vacancies. $^{51,70,71,74}$ Both dimer, $\left(\mathrm{Ce}_{\mathrm{Ce}}^{\prime}-\mathrm{V}_{\mathrm{O}}^{\bullet \bullet}\right)^{\bullet}$, and trimer, $\left(\mathrm{Ce}_{\mathrm{Ce}}^{\prime}-\mathrm{V}_{\mathrm{O}}^{\bullet \bullet}-\mathrm{Ce}_{\mathrm{Ce}}^{\prime}\right)^{\times}$species have been considered, and the preponderance of the data, both experimental and computational (see Fig. 3), indicate the predominance of trimers, reflecting another departure from early interpretations. In many computational studies, the presence of $\left(\mathrm{Ce}_{\mathrm{Ce}}^{\prime}-\mathrm{V}_{\mathrm{O}}^{\bullet \bullet}-\mathrm{Ce}_{\mathrm{Ce}}^{\prime}\right)^{\times}$ is in fact unremarkable and treated as entirely expected. ${ }^{65,73,75,76}$

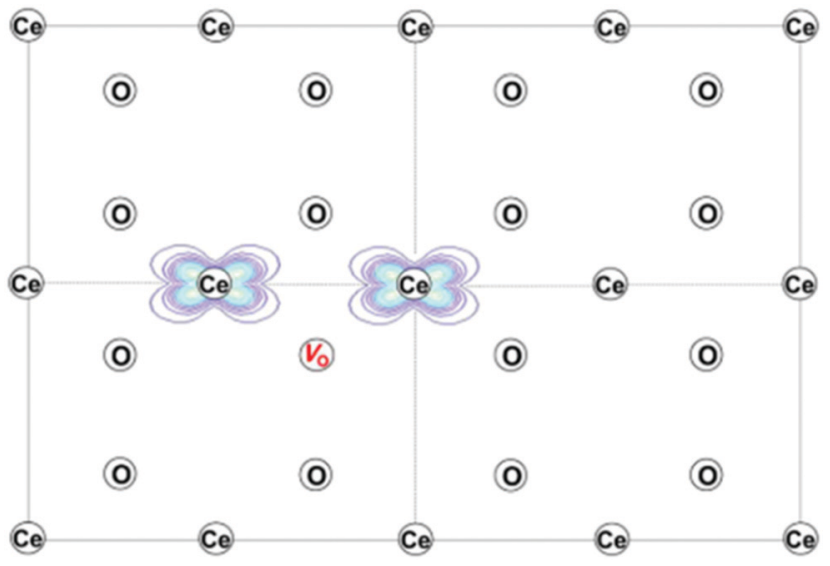

Fig. 3 Projection of the computed charge density of reduced ceria on the (110) plane, showing the electronic ground state with localization of two $\mathrm{Ce}^{3+}$ ions next to an oxygen-vacancy, to create the trimer associate. Reproduced from ref. 73 with permission from American Chemical Society, copyright 2016

From a global defect chemical perspective, $\mathrm{V}_{\mathrm{O}}^{\bullet}$ and $\left(\mathrm{Ce}_{\mathrm{Ce}}^{\prime}-\mathrm{V}_{\mathrm{O}}^{\bullet \bullet}\right)^{\bullet}$ are qualitatively the same, just as $\mathrm{V}_{\mathrm{O}}^{\times}$and $\left(\mathrm{Ce}_{\mathrm{Ce}}^{\prime}-\mathrm{V}_{\mathrm{O}}^{\bullet \bullet}-\mathrm{Ce}_{\mathrm{Ce}}^{\prime}\right)^{\times}$ are. However, the local configurations are quite distinct. ${ }^{71}$

Beyond the types of associates described above, some evidence has been put forward for the occurrence of more extended defect clusters, including double-trimers (hexamers) that emerge from a treatment of the experimental thermogravimetric data, ${ }^{56}$ and oxygen-vacancy pairs that emerge from $a b$ initio calculations. ${ }^{73,75,76}$ Not surprisingly, the local configurational and macroscopic computational complexity increases dramatically when such extended defects are fully treated, without providing (due to their low concentrations) a qualitatively different picture of the macroscopic transport and nonstoichiometry behavior. Detection of such defects, which are increasingly recognized to impact other types of properties, is best achieved by the local probes discussed in the next chapters.

Returning to the specific defect chemical reactions of Table 1, when the concentrations of species are sufficiently low, chemical activity is adequately represented by concentration, and mass action laws (eqn (2) and (4)) are appropriately expressed in terms of concentration (as written). At small $\delta$, at which $\left[\mathrm{Ce}_{\mathrm{Ce}}^{\times}\right]$and $\left[\mathrm{O}_{\mathrm{O}}^{\times}\right]$are $\sim 1$, eqn (4) implies that the power law exponent describing the variation in trimer concentration with oxygen partial pressure is $-\frac{1}{2}$. The total vacancy concentration will be the sum of the isolated vacancy concentration and the trimer concentration, eqn (10), giving rise to a slope in the double-logarithmic plot of $\delta$ vs. $p \mathrm{O}_{2}$ at moderate $\delta$ that falls between $-\frac{1}{6}$ and $-\frac{1}{2}$. Such a result is experimentally observed, Fig. $4 .^{50,51,74}$

A more complete treatment of the defect equilibrium behavior considers the reduction reactions in tandem, as well as site occupancy restrictions. The latter constrain the total concentration of the anionic and cationic species to the number of sites available (for a total fractional concentration of 1 on each 
(a)

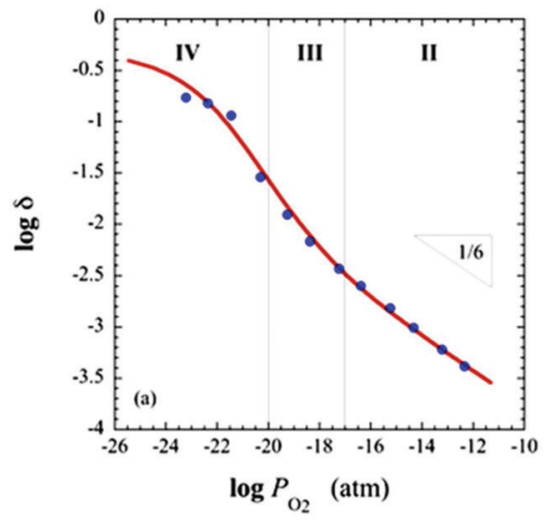

(b)

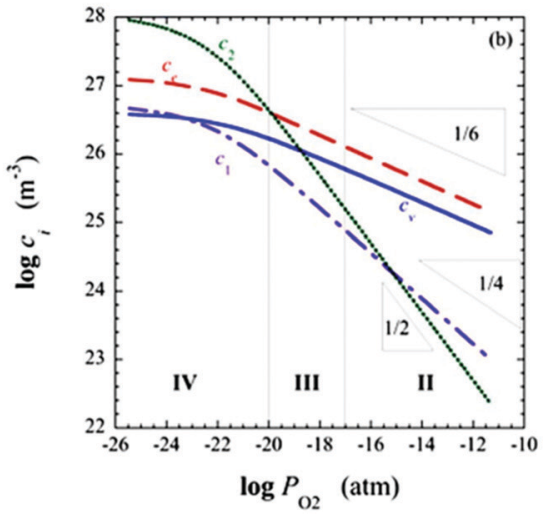

Fig. 4 Defect chemical modeling of the oxygen partial pressure dependence of non-stoichiometry in (undoped) ceria: (a) comparison of the data from Panlener ${ }^{50}$ to the model results; and (b) individual defect, concentrations, where $C_{v}$ refers to unassociated oxygen vacancies, $C_{e}$ to unassociated polarons, $C_{1}$ to dimers $\left(\mathrm{Ce}_{\mathrm{Ce}}^{\prime}-\mathrm{V}_{\mathrm{O}}^{\bullet \bullet}\right)^{\bullet}$, and $C_{2}$ to trimers $\left(\mathrm{Ce}_{\mathrm{Ce}}^{\prime}-\mathrm{V}_{\mathrm{O}}^{\bullet \bullet}-\mathrm{Ce}_{\mathrm{Ce}}^{\prime}\right)^{\prime}$. Slopes shown in (b) are guides to the eye. Modeling reveals three regions of defect behavior: in II isolated vacancies (and isolated polarons) dominate, and the power law exponent is close to $-\frac{1}{6}$; in III trimers are comparable in importance to isolated defects and the dependence of non-stoichiometry on oxygen partial pressure rises; in IV site saturation effects emerge. Reproduced from ref. 74 with permission from AIP publishing, copyright 2007.

type of site), eqn (7) and (8). Bishop et al. employed this framework to evaluate the oxygen partial pressure dependent non-stoichiometry data at $800{ }^{\circ} \mathrm{C}$. The numerically implemented fit reproduces the experimental results well, Fig. 4(a). Due to the inclusion of site restriction effects, the model reproduces the plateau in $\delta$ that occurs in the vicinity of 0.5 , where the stoichiometry approaches $\mathrm{Ce}_{2} \mathrm{O}_{3}$. The analysis furthermore reveals the concentration of each type of species, Fig. 4(b), and yields values of the equilibrium constants, $K_{\mathrm{r} \text {,isolated }}$ and $K_{\mathrm{r} \text {,trimer. }}{ }^{51}$

An alternative methodology for treating the redox thermodynamics considers the macroscopic behavior without (immediate) reference to the nature of the defects formed. ${ }^{49,50}$ The reduction of the undoped material by this formalism is written for an infinitesimal $\varepsilon$, change in state as

$$
\lim _{\varepsilon \rightarrow 0} \frac{1}{\varepsilon} \mathrm{CeO}_{2-\delta}=\lim _{\varepsilon \rightarrow 0} \frac{1}{\varepsilon} \mathrm{CeO}_{2-\delta-\varepsilon}-\frac{1}{2} \mathrm{O}_{2} .
$$

Noting that the chemical potentials of the two solids are identical in the limit $\varepsilon \rightarrow 0$, the equilibrium constant, $K_{\text {red }}$ for this reaction, becomes

$$
K_{\text {red }}(\delta)=\hat{p}_{\mathrm{O}_{2}}^{\frac{1}{2}}=\exp \left(\frac{-\Delta_{\text {red }} G^{\circ}(\delta)}{R T}\right)
$$

where $R$ and $T$ are the universal gas constant and temperature, respectively, $\hat{p}_{\mathrm{O}_{2}}$ is the oxygen pressure relative to the standard oxygen pressure of $1 \mathrm{~atm}$ (see Table 1 ), and $\Delta_{\text {red }} G^{\circ}$ is the standard Gibbs energy of reduction (per mole of $\mathrm{O}$ ). This equality expresses the value of the oxygen partial pressure that generates an equilibrium non-stoichiometry of $\delta$ at a given temperature, and reflects the fact that, under equilibrium, the partial molar Gibbs energy of oxygen in the solid must be equal to that in the gas.

The equilibrium reduction constant of eqn (13), must of course, be related to the mass action constants of Table 1 (eqn (2) and (4)). If isolated defects dominate the reduction at low $\delta$ and associates dominate the behavior as $\delta$ increases, then it is reasonable to expect that $K_{\text {red }}$ will be a function of nonstoichiometry, gradually changing in value from that defined by $K_{\mathrm{r} \text {,isolated }}$ to that defined by $K_{\mathrm{r} \text {,trimer }}$. Considering first the limit of very small $\delta$, the globally and microscopically defined equilibrium constants differ by the term

$$
\frac{K_{\mathrm{r}, \text { isolated }}}{K_{\mathrm{red}}}=\frac{\left[\mathrm{V}_{\mathrm{O}}^{\bullet \bullet}\right]\left[\mathrm{Ce}_{\mathrm{Ce}}^{\prime}\right]^{2}}{\left[\mathrm{Ce}_{\mathrm{Ce}}^{\times}\right]^{2}\left[\mathrm{O}_{\mathrm{O}}^{\times}\right]}=\Omega
$$

This quantity specifies the number of distinguishable ways to distribute the defects. Recognizing $R \ln \Omega$ as $S_{\text {config, the configura- }}$ tional entropy associated with the presence of the defects, it becomes apparent that $\Delta_{\text {red }} S_{\text {defect }}^{\circ}$, the entropy for the defect reaction eqn (1), and by analogy that for eqn (3) also, is related to that for the macroscopic reduction of eqn (13), $\Delta_{\text {red }} S^{\circ}$, according to

$$
\Delta_{\text {red }} S_{\text {defect }}^{\circ}=\Delta_{\text {red }} S^{\circ}-S_{\text {config }},
$$

where $S_{\text {config }}$ is clearly a function of $\delta$. It can be shown that ${ }^{77}$

$$
\Delta_{\text {red }} S^{\circ}=S_{\text {config }}+\Delta_{\text {red }}^{\text {excess }} S^{\circ}+\frac{1}{2} S_{\mathrm{O}_{2}}{ }^{*}
$$

where $S_{\mathrm{O}_{2}}{ }^{*}$ is the large entropy of gas phase oxygen at standard pressure and $\Delta_{\text {red }}^{\text {excess }} S^{\circ}$ is the non-configurational solid state entropy, a typically small term sometimes called the excess entropy. ${ }^{78}$ It is to be noted that although the enthalpy terms discussed in the microscopic and macroscopic approaches are directly equal to one another, the entropy terms differ by the configurational entropy.

Taking into account the observation that associates become increasingly important as $\delta$ increases, it is reasonable to expect that the macroscopic $K_{\text {red }}$ will be a function of non-stoichiometry, gradually changing in value from that defined by $K_{\mathrm{r} \text {,isolated }}$ to that defined by $K_{\text {r,trimer }}$ Thus, one can anticipate that the enthalpy and entropy terms associated with $K_{\text {red }}$ will also gradually vary from those describing the isolated defects to those 
describing associates. In particular, because the energetics associated with the two very distinct defect reactions eqn (1) (isolated defects) and (3) (trimers) differ significantly, as shown for example, by DFT calculations, ${ }^{71}$ a dependence of $\Delta_{\text {red }} H^{\circ}$ on $\delta$ is fully expected. In contrast, the excess entropy may be relatively invariant with non-stoichiometry.

Experimental determination of the enthalpy and entropy of reduction requires reliable thermogravimetric data spanning a wide range in $T-\hat{p}_{\mathrm{O}_{2}}$ space. Following the microscopic approach, one then makes use of the expressions in Table 1 to derive from these data the equilibrium constants for the specific defect reactions of interest at multiple temperatures. In the macroscopic, model-free approach, one extracts effective equilibrium constants at multiple, specified values of $\delta,\left\{\delta_{i}\right\}$, and for each $\delta_{i}$, at multiple temperatures. In both cases, extraction of the enthalpy and entropy terms relies on the linearity of the data when presented as Arrhenius plots of the equilibrium constants. Such linearity implies the temperature independence of the reduction enthalpies and entropies, behavior that is sometimes assumed without verification.

Despite these challenges, quantification of the redox characteristics of bulk ceria has been rather successful. From an analysis of the thermogravimetric data of Panlener ${ }^{50}$ within the context of the microscopic model, Tuller and Nowick ${ }^{68}$ concluded that the enthalpy of reduction in the isolated-defect regime is $451 \pm 7 \mathrm{~kJ} \mathrm{~mol}^{-1}$. The associated-defect regime was analyzed assuming predominance of singly ionized oxygen vacancies. The authors extracted an enthalpy of $397 \mathrm{~kJ} \mathrm{~mol}^{-1}$, and hence even an imperfect defect model (it is now known that the association is dominated by neutral trimers) reveals the fact that defect association decreases the enthalpy of reduction. Using the macroscopic approach, several studies have shown that the enthalpy of reduction of ceria decreases monotonically from a value of $\sim 475 \mathrm{~kJ} \mathrm{~mol}^{-1}$ at small $\delta$, to $\sim 400 \mathrm{~kJ} \mathrm{~mol}^{-1}$ at $\delta \sim 0.1 .^{48-50,79-82}$ Thus, the microscopic and macroscopic approaches both reveal that the enthalpy decreases with increasing $\delta$, and the former provides a chemical rational for this trend.

A notable exception to the overall agreement in the literature lies in the work of Takacs et al.,${ }^{47}$ who recently re-evaluated the defect-chemistry in the context of thermochemical fuel production in the temperature range of 1300-1500 ${ }^{\circ} \mathrm{C}$. While these authors reported a monotonically decreasing enthalpy of reduction with $\delta$ (consistent with the prior studies), the absolute value of the enthalpy at small $\delta$ was substantially higher than obtained in previous reports, see Fig. 5. For the purposes of evaluating the potential of ceria for fuel production via high temperature redox cycles, Takacs et al. ${ }^{47}$ measured even small non-stoichiometry at $1300-1500{ }^{\circ} \mathrm{C}$. Thus, as suggested by the authors, the discrepancy in absolute enthalpy with respect to the prior literature is likely due to a temperature dependence of this quantity. Notwithstanding this outlying result, there is generally a good understanding of the origin of the enthalpy variation with $\delta$ at small to moderate values of $\delta$ in $\mathrm{CeO}_{2-\delta}$.

At large $\delta$ a substantial disagreement between the microscopic and macroscopic approaches appears. Whereas the

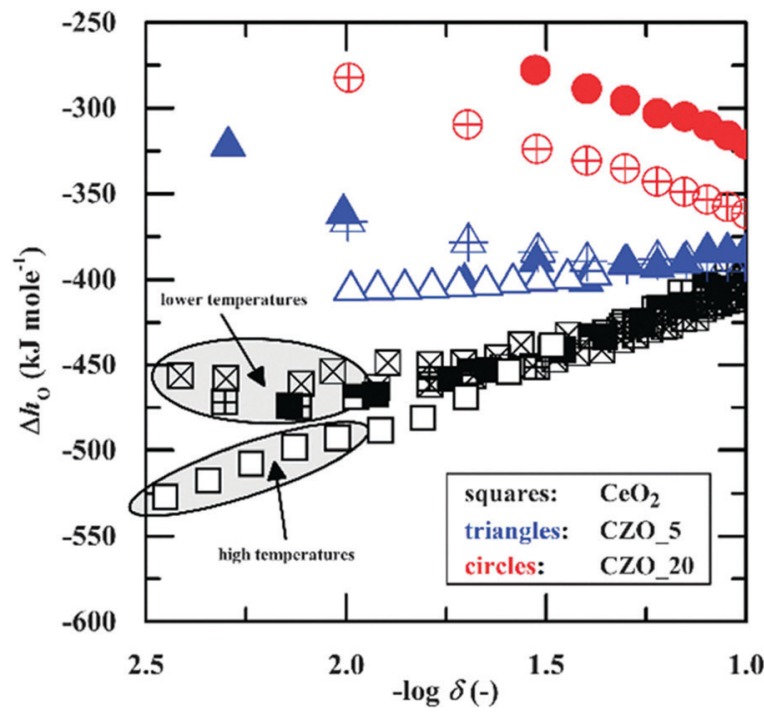

Fig. 5 Enthalpy of oxidation of ceria ( $\mathrm{kJ}$ per mol-O) as a function of non-stoichiometry. Blue and red data markers refer to values for 5 and 10 mol\% Zr substituted ceria (denoted as CZO and according number for dopant molar concentration), respectively, and are not considered here. The magnitude of the enthalpy decreases with increasing $d$. High temperature measurements yield higher enthalpy values (in magnitude) than low temperature measurements. Reproduced from ref. 47, creative commons license 2015 .

microscopic approach, when limited to defect reactions 1 and 3 , inherently predicts a monotonic decrease in the enthalpy of reduction with increasing $\delta$, analysis of the thermogravimetric data within the macroscopic framework shows that the enthalpy rises beyond $\delta \sim 0.15$ (reaching $\sim 410 \mathrm{~kJ} \mathrm{~mol}^{-1}$ at $\delta=0.28$ ). Reflecting this disagreement, the model fit for a temperature of $800{ }^{\circ} \mathrm{C}$ presented by Bishop et al. using the microscopic defect chemical approach noticeably deviates from the experimental data, under-predicting the vacancy concentration when $\delta \gtrsim 0.1 .^{51,56}$ The probable explanation for a peak in the enthalpy of reduction at stoichiometry $\mathrm{CeO}_{1.81}$ is the onset of a degree of ordering within the single-phase fluorite lattice structure, not accounted for in typical microscopic models. Pair distribution function analysis in conjunction with reverse Monte Carlo modeling of high-resolution neutron powder diffraction data collected at $1000{ }^{\circ} \mathrm{C}$ has indeed revealed the presence of oxygen-vacancy pairs with preferential $\langle 111\rangle$ alignment for ceria of similar oxygen content within the nominally fluorite structure. ${ }^{83}$ The vacancy arrangement is reminiscent of that encountered in the vacancy ordered phases $\mathrm{Ce}_{11} \mathrm{O}_{20}$ and $\mathrm{Ce}_{7} \mathrm{O}_{12} \cdot{ }^{84}$

Until recent years, the entropy of reduction of ceria has received somewhat lesser attention than the enthalpy. The experimental data agree that the excess entropy of eqn (15) is small, ${ }^{50}$ but nevertheless the solid state reduction entropy, $\Delta_{\text {red }} S^{\circ}-\frac{1}{2} S_{\mathrm{O}_{2}}{ }^{*}$ is large enough to render ceria a benchmark material for thermochemical fuel production via redox cycling. ${ }^{15,16}$ The early literature on ceria assumed the excess entropy was dominated by vibrational effects, as is typical in solid state systems. Recent computational studies suggest that 
in fact electronic effects, related to the high density of electronic states in the narrow $4 \mathrm{f}$ band, may be responsible for the large solid state reduction entropy of ceria. ${ }^{85}$ The results furthermore caution against parsing the electronic defect contributions to the entropy into configurational and non-configurational terms. The relevance of this behavior to the local defect structure remains to be explored but is potentially significant.

The defect chemical framework, which has been largely successful in describing the redox behavior of bulk, undoped ceria, can be readily applied to doped compositions, where we focus here again on trivalent dopants indicated with overall stoichiometry $\mathrm{Ce}_{1-x} \mathrm{Do}_{x} \mathrm{O}_{2-x / 2-\delta}$. As noted above, oxygen vacancies resulting directly from acceptor-doping are designated 'extrinsic' and those resulting from reduction are designated 'intrinsic'. When $\delta$ is small $\left(\delta \ll\left[\mathrm{Do}_{\mathrm{Ce}}^{\prime}\right]\right)$ the concentration of extrinsic vacancies is by definition small, and the electroneutrality condition, eqn (6), can be approximated as

$$
\left[\mathrm{V}_{\mathrm{O}}^{\bullet \bullet}\right] \approx\left[\mathrm{V}_{\mathrm{O}}^{\bullet \bullet}\right]^{\mathrm{ext}}=\frac{1}{2}\left[\mathrm{Do}_{\mathrm{Ce}}^{\prime}\right]
$$

This result, along with eqn (11), implies that the polaron concentration and hence also the intrinsic vacancy concentration in doped ceria will vary with oxygen partial pressure according to a power law exponent of $-\frac{1}{4}$, in contrast to the $-\frac{1}{6}$ expected for undoped ceria in the low $\delta$ limit.

In a broad sense, the experimental thermogravimetric data agree with the predictions of ideal solution behavior in trivalent doped ceria. ${ }^{49,51,77,86}$ However, because of the difficulty of accurately measuring $\delta$ by gravimetry when the $\delta \lesssim 10^{-3}$, it is unreasonable to accept thermogravimetric results alone as definitive proof of the defect chemical model. In contrast, conductivity is easily measured in any regime including that producing a low $\delta$, and a power law exponent of $-\frac{1}{4}$ relating conductivity and oxygen partial pressure is routinely reported for doped ceria, ${ }^{87-89}$ as shown for example in Fig. 6. It is to be emphasized that the power law exponent of $-\frac{1}{4}$ is observed not because the total vacancy concentration is changing, but because the concentration of electrons, which have higher mobility than vacancies, is changing. In light of such experimental results, the high-temperature defect chemical behavior in the dopant dominated-region is largely considered settled. However, it must be noted that charge-neutral associates formed of an oxygen vacancy, a dopant ion, and an electronic defect, so-called mixed trimers, would also lead to an exponent of $-\frac{1}{4},{ }^{51}$ and the occurrence of such species, possibly even in large concentrations, cannot be ruled out on the basis of the power law exponent alone.

Beyond power law exponents, much as with undoped ceria, the measured $\delta\left(p \mathrm{O}_{2}\right)$ for doped ceria have been used to determine defect equilibrium constants, evaluate the thermodynamic parameters, and further test the validity of defect chemical models describing the redox behavior. In particular, Bishop has presented a comprehensive study of the behavior of $\mathrm{Ce}_{0.9} \mathrm{Gd}_{0.1} \mathrm{O}_{1.95-\delta}{ }^{51}$ whereas Hashimoto and coworkers have evaluated both Gd and Sm-doped ceria, at both 10 and $20 \mathrm{~mol} \%$ doping levels. ${ }^{49,77,86}$ These dopants generally lower the enthalpy of reduction, relative to that in undoped

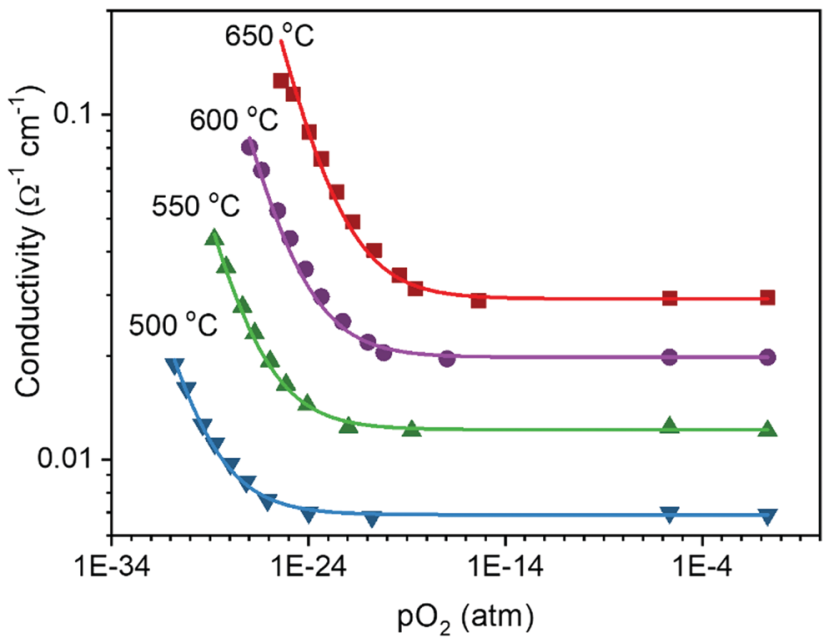

Fig. 6 Electrical conductivity of 15 mol\% Sm doped ceria as a function of oxygen partial pressure at the temperatures indicated. The solid lines are fits to the expression $\sigma_{\text {tot }}=\sigma_{\text {ion }}+\sigma_{\text {eon }}^{0} \times p\left(\mathrm{O}_{2}\right)^{-\frac{1}{4}}$. Under reducing conditions, a power law exponent describing the dependence of conductivity on oxygen partial pressure of $-\frac{1}{4}$ is obtained, consistent with ideal solution behavior. Adapted from ref. 88 with permission from Wiley, copyright 2005.

ceria and decrease the dependence of the enthalpy on nonstoichiometry. Doping with calcium produces a similar transition from non-ideal to ideal solution thermodynamics, i.e., from $\delta$ dependent to $\delta$-independent enthalpy. ${ }^{90}$ Given the presence of a large concentration of extrinsic vacancies in doped systems, the tendency towards ideal solution behavior (which implies weaker defect-defect interactions) is surprising on first sight. However, ideal solution behavior here means, in fact, a transition from reduction of ideal, isolated defects according to eqn (1), to reduction of 'ideal' associates according to eqn (3). Viewed in that context, the onset of ideal behavior with an enthalpy of reduction that is independent of $\delta$ is reasonable. At large $\delta$, the onset of a regime in which the non-stoichiometry is relatively insensitive to oxygen partial pressure has been attributed to site restriction effects (eqn (7)-(9)) and the emergence of defect ordering, much as has been suggested for undoped ceria. ${ }^{51,56}$ The local structure methods described in this review have the potential to resolve these apparent discrepancies.

A key distinction between undoped and doped ceria arises at moderate temperatures and ambient pressures. Under such conditions, the defect concentration in ideally pure ceria approaches zero, whereas in doped ceria eqn (17) continues to apply. However, the extrinsic vacancies generated by doping can become associated with the dopant species. A convenient, first-order formalism for treating such effects in dilute systems is to express trapping according to the reaction ${ }^{91}$

$$
\mathrm{V}_{\mathrm{O}}^{\bullet \bullet}+\mathrm{Do}_{\mathrm{ce}}^{\prime} \leftrightarrow\left(\mathrm{V}_{\mathrm{O}}^{\bullet \bullet}-\mathrm{Do}_{\mathrm{ce}}^{\prime}\right)^{\bullet}
$$

with equilibrium constant

$$
K_{\text {trap }}=\frac{\left[\mathrm{V}_{\mathrm{O}, \text { free }}^{\bullet \bullet}\right]\left[\mathrm{Do}_{\mathrm{Ce}, \text { free }}^{\prime}\right]}{\left[\mathrm{Do}_{\mathrm{Ce}, \text { trap }}^{\prime}\right]} .
$$


Due to the low cation mobility at the temperatures at which associate formation is thermodynamically driven, appreciable concentrations of the neutral defect $\left(\mathrm{Do}_{\mathrm{ce}}^{\prime}-\mathrm{V}_{\mathrm{O}}^{\bullet \bullet}-\mathrm{Do}_{\mathrm{ce}}^{\prime}\right)$ do not occur. In the state of maximum association, typically corresponding to temperatures of $\sim 300{ }^{\circ} \mathrm{C}$ and lower, charge and mass balance requirements imply that half the dopant ions will form associates, trapping all of the available oxygen vacancies, with the remainder of the dopant species remaining isolated. Furthermore, as has been pointed out, at a dopant concentration of just 2 at\%, already $30 \%$ of the dopant species in a randomly doped ceria host will have a dopant in its nearest neighbor or next nearest neighbor cation shell. ${ }^{92}$ Thus, the system can only be treated as dilute for very low dopant concentrations.

Experimentally, dopant-vacancy association has been inferred from ionic conductivity measurements, specifically, from a difference in high and low temperature activities for charge transport and from the existence of a conductivity maximum, as well as a minimum in low temperature activation energy, as a function of dopant concentration. ${ }^{92-95}$ The temperature dependence of the activation energy results because the vacancies must be thermally activated out of the trap sites. Hence, the activation energy measured at low temperature is a sum of migration and trapping terms, whereas at high temperature, at which all vacancies are liberated from trapping sites, the activation energy is due only to migration effects. Thus, values for the trapping and migration energies can in principle be determined from the difference in the activation energies between the two regions, or more rigorously from the temperature dependence of the apparent activation energy across the regions.

Not immediately predicted by the first order model, but widely observed, is a strong dependence of the empirical activation energy for ion transport on dopant concentration. Specifically, the first order model predicts that in the deeply associated region, in which $\left[\mathrm{Do}_{\mathrm{Ce}, \mathrm{free}}^{\prime}\right] \approx\left[\mathrm{Do}_{\mathrm{Ce}, \text { trap }}^{\prime}\right] \approx$ $\frac{1}{2}\left[\mathrm{Do}_{\mathrm{Ce}, \text { total }}^{\prime}\right]$, the free (mobile) vacancy concentration is given from eqn (19) by $\left[\mathrm{V}_{\mathrm{O} \text {, free }}^{\bullet \bullet} \approx K_{\text {trap }}\right.$, and is independent of dopant concentration. ${ }^{92}$ Thus, in order for a dependence on dopant concentration in the activation energy to emerge, either the vacancy trapping energy, or the vacancy migration energy, or both, must depend on the dopant concentration. In light of the relative proximity of dopant species to one another even at very low doping levels, a dependence of the trapping and migration energetics on dopant concentration is not surprising. On the other hand, the complexity of the problem, with a myriad of possible vacancy-dopant configurations, implies that macroscopic conductivity measurements alone do not permit reliable decomposition of the energy contributions to the observed values.

A treatment of the problem of the ionic conductivity maximum in doped ceria has been approached more recently computationally, with an excellent overview reported by Martin and coworkers in 2018. ${ }^{32}$ By such methods it is possible to differentiate between effects due to trapping of vacancies in defect states and those due to impacts on the overall migration

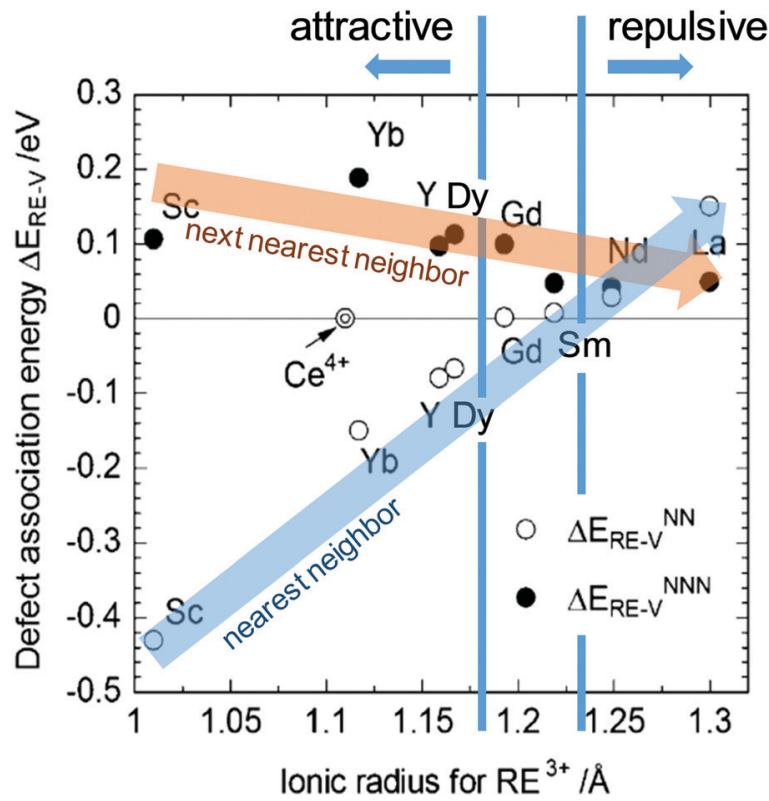

Fig. 7 Association energy of dopant ion with vacancies as nearest and next nearest neighbors, plotted as function of dopant $\left(\mathrm{RE}^{3+}\right)$ radius. Adapted from ref. 96 with permission from Royal Society of Chemistry, copyright 2009.

barriers. The former, which establish the equilibrium concentration of defects at a given temperature and doping level, are of particular interest here. Shown in Fig. 7 are DFT (density functional theory) computed association energies for vacancy trapping in doped ceria as a function of dopant species, specifically, as a function of the ionic radius of the rare earth (RE) element. ${ }^{96}$ For placement of the vacancy in a nearest neighbor site (see Fig. 10 below for the structural arrangement), there is a clear trend in association energy with radius, with elements smaller than Gd preferentially trapping the vacancy at this position, and those larger than Sm repelling the vacancy. The dopants Gd and Sm, known to produce high ionic conductivity in ceria, are neither attractive nor repulsive towards vacancies. A weaker and opposite trend is evident for the next nearest neighbor site, and in this case the trapping is apparently never favored. Significantly, the computation further predicts (not shown) that the magnitude of the defect association energy strongly increases with the number of nearest neighbor RE ions surrounding the vacancy, explaining, in part, the concentration dependence of the ionic conductivity. Thus, computational studies are beginning to shed important light on the local configurations of dopants and oxygen vacancies in doped ceria, although details remain unresolved, and may ultimately depend on processing and the influence of quenching on the cation distributions in the solid.

A final area of open questions about the defect-chemistry of ceria lies in the realm of the interfacial properties. Both the internal and external interfaces (i.e., grain boundaries and surfaces, respectively) display strong evidence of enhanced reduction relative to the bulk. For example, thermogravimetric measurements of high surface area ceria, both undoped ${ }^{97}$ and 


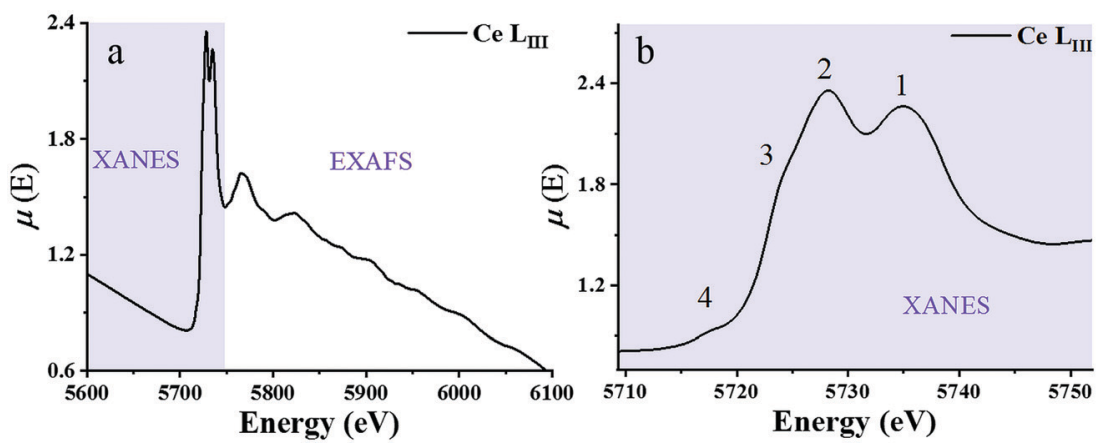

Fig. 8 (a) X-ray absorption spectrum at the $\mathrm{Ce}$ - $\mathrm{L}_{\text {III }}$ edge for $\mathrm{CeO}_{2}$ powder described in ref. $59 \mu(E)$ denotes the X-ray absorption coefficient. XANES and EXAFS regions are indicated. (b) The expanded XANES range of the absorption spectrum, with four main features labeled 1-4 described in the text. Adapted from ref. 45 with permission from Elsevier, copyright 2010

$10 \mathrm{~mol} \%$ Gd-doped, ${ }^{56}$ reveal higher and less oxygen partial pressure dependent non-stoichiometry relative to conventional samples with large particle sizes. ${ }^{56,97,98}$ The immediate conclusion is that the oxygen-vacancy and $\mathrm{Ce}^{3+}$ concentration is enhanced at the surfaces. Studies of surface oxidation states by near-ambient pressure XPS measurements have directly proven such surface enhancement. ${ }^{10,11,99}$ Similarly, grain boundaries are often described as positively charged, implying an enhanced vacancy concentration at the grain boundary core, which is charge balanced by a negative space charge region. Within this region, positively charged defects, in particular oxygen vacancies, are depleted. ${ }^{100,101}$ Support for the space charge model of grain boundaries in ceria derives, in part, from impedance spectroscopic measurements ${ }^{101}$ in which bulk and grain boundary resistances can be deconvoluted. Because the spatial extent of the space charge region scales with $c_{\mathrm{Do}}{ }^{-1 / 2}$, where $c_{\mathrm{Do}}$ is the volumetric dopant concentration, ${ }^{100,102}$ space charge effects are particularly important in lightly or undoped ceria, as has been observed experimentally. ${ }^{101,102}$ The spectroscopic methods reviewed below, which provide access to spatially resolved information about oxidation state and coordination environment, appear well suited for going beyond the inferences from defect model and transport models to enable the unraveling of outstanding questions regarding defect interactions in ceria.

\section{Local structure of ceria solid solutions as probed by X-ray absorption spectroscopy}

\subsection{Introduction to X-ray absorption spectroscopy}

X-ray absorption spectroscopy (XAS) is a synchrotron-based technique that provides element-specific information about local atomic and electronic structure of materials. In this method, X-ray photons excite core-level electrons that interact with the nearest neighboring (NN) atoms to the absorbing species. In a typical XAS experiment, the intensity $\left(I_{t}\right)$ of the $\mathrm{X}$-ray beam transmitted through the sample is measured along with the incident beam intensity $I_{0}$ as a function of X-ray energy, $E$. The X-ray absorption coefficient $\mu(E)$ is given by the
Beer-Lambert law as $\mu \cdot d=\ln \left(I_{0} / I_{\mathrm{t}}\right)$, where $d$ is the path of the beam through the material (or the sample thickness). A typical XAS spectrum for ceria powder is shown in Fig. 8, taken from ref. 45. A sharp increase in $\mu(E)$ occurs when the incident X-ray photon carries sufficient energy to excite an electron from a low-energy bound state in the atom to an upper energy level. This element specific increase is termed an "absorption edge", and is labeled $\mathrm{K}, \mathrm{L}$ or $\mathrm{M}$, depending on the principal quantum number $n$ of the initial state from which the excitation occurred. Namely, K corresponds to the 1s state, L (subscripts I-III) corresponds to $2 \mathrm{~s}, 2 \mathrm{p}_{1 / 2}$ and $2 \mathrm{p}_{3 / 2}$ states, while $\mathrm{M}$ (subscripts I-V) corresponds to $3 \mathrm{~s}, 3 \mathrm{p}_{1 / 2}, 3 \mathrm{p}_{3 / 2}, 3 \mathrm{~d}_{3 / 2}$ and $3 \mathrm{~d}_{5 / 2}$ states. ${ }^{103}$ In Fig. 8, the Ce- $\mathrm{L}_{\mathrm{III}}$ absorption edge in $\mathrm{CeO}_{2}$ powder appears as a sharp increase in absorption at $\sim 5724 \mathrm{eV}$ and is due to the transition $2 \mathrm{p}_{3 / 2} \rightarrow 5 \mathrm{~d}_{5 / 2} .{ }^{104}$ Measurements of the Ce$\mathrm{L}_{\mathrm{III}}$ edge are usually chosen for undoped and doped ceria, due to the scarcity of beamlines that can access the high energy of the Ce-K edge $(\sim 40450 \mathrm{eV}) \cdot{ }^{105-107}$ The negative slope of the background in Fig. 8 is captured in the Victoreen formula, $\mu(E) \sim 1 / E^{3}$. Measurements can be performed in either transmission or fluorescence mode. The latter is necessitated when the sample is either too thick for transmission, or the concentration of the X-ray absorbing species is too dilute. Fluorescence detection is also beneficial for thin film samples, as it minimizes the signal of the substrate. It is important to note that XAS probes 'bulk' properties, where penetration depth is a function of the material X-ray attenuation coefficient at the relevant incident $\mathrm{X}$-ray energy. For example, probing the Ce- $\mathrm{L}_{\text {III }}$ edge at $\sim 6 \mathrm{keV}$ in ceria or doped ceria compounds yields an attenuation length (where the intensity of radiation falls to $1 / e$ of that at the material surface) of 5-10 $\mu \mathrm{m} .{ }^{108,109}$ The penetration depth increases with the incident energy in proportion to the decrease in absorption given by the Victoreen formula above. It is possible however to probe surface properties (few $\mathrm{nm}$ penetration depth) using XAS by setting a grazing incident configuration, where the incident angle is below the angle for total external reflection for the specific material.

Historically, XAFS (the fine features emerging in the absorption spectrum) has been divided into two X-ray energy regions: the X-ray absorption near edge structure (XANES) and the extended XAFS (EXAFS). The XANES portion of the spectrum is sensitive 
to the redox state. It enables quantification of $\mathrm{Ce}^{3+} v s . \mathrm{Ce}^{4+}$ valence states and reveals the effect of dopant cations and grain size on the redox chemistry of ceria-based materials. A XANES spectrum includes features from $c a .40 \mathrm{eV}$ below to $c a .50 \mathrm{eV}$ above the absorption edge, the most prominent being the socalled "white line" which appears as a strong peak, positioned at the absorption maximum. (See ref. 110-113 for XANES theory.) The Ce- $\mathrm{L}_{\text {III }}$ white line displayed in Fig. 8 reveals four prominent features, which are understood to result from a combination of several effects. Many-body final states (due to mixing of $4 \mathrm{f}^{0}$ and $4 \mathrm{f}^{1} \mathrm{~L}$ final configurations where $\mathrm{L}$ represents a hole in the delocalized $\mathrm{O} 2 \mathrm{p}$ band of the ligand oxygen species) give rise to the main peak splitting (generating peaks 1 and 2). Crystal field splitting of the $5 \mathrm{~d}$ states $\left(\Delta_{\mathrm{f}} \approx 3.7 \mathrm{eV}\right)$ gives rise to the shoulder feature denoted as 3 . This shoulder also overlaps the white line due to absorption by reduced $\mathrm{Ce}^{3+}$ species. ${ }^{104,114}$ In high-resolution experiments, examples of which are described below, peaks 2 and 3 are fully resolved, and the splitting of peak 1, again due to crystal field effects, can also be observed. ${ }^{115}$ Multiple scattering, which provides access to final states at the bottom of the $\mathrm{Ce} 5 \mathrm{~d}$ conduction band with delocalized $d$ character, gives rise to the pre-edge feature labeled 4. XANES spectra are thus rich with chemical and structural information. From the positions and relative intensities of peaks 1 and 2 information about local geometry, the number of, and the degree of order in coordinating ligands about the central Ce absorber can be gained..$^{37,38,116}$ Similarly, analysis of the relative intensity of peak/shoulder 3 can be used to track the extent of reduction of ceria-based materials. ${ }^{117-120}$

The EXAFS region of the absorption coefficient (which is the focus of the XAFS portion of this review) extends to $\mathrm{ca}$. 1000$1500 \mathrm{eV}$ above the absorption edge and provides information about nearest neighbor bonding environments, such as the coordination numbers, interatomic distances and their disorder. In ceria and other related materials, in which $\mathrm{L}_{\mathrm{III}}$ edges are used to measure EXAFS, the useful energy range is limited by the presence of $\mathrm{L}_{\mathrm{II}}$ edges and can span as little as $300-400 \mathrm{eV}$ (EXAFS region indicated in Fig. 8a). EXAFS features originate from the interference effect ${ }^{110-113}$ caused by the interaction of the outgoing photoelectron waves ejected from inner core levels by the incoming X-ray radiation, and those scattered back to the absorbing atom by its neighbors. This interference modulates the wavefunction of the final state and, therefore, the probability of absorbing the X-ray photon, according to Fermi's golden rule. This modulation, appearing in the high energy portion of the X-ray absorption spectrum, was recognized by Stern et $a .^{121}$ as being related to the radial distribution of neighboring atoms around the central absorbing atom. Due to the nature of the photoelectron wave interference that originates from the path difference between the outgoing and backscattered waves, the interference can be constructive or destructive (and thus the fine structure of the absorption coefficient will exhibit a maximum or a minimum), depending on the number (integer or fractional) of wavelengths in the total photoelectron path. For single-scattering events, the path is equal to twice the distance between the absorber and a nearest neighbor atom. ${ }^{122}$ The information about the environment of the absorber (the number of nearest neighbors, their identities, distances to the absorber and the spread in these distances) can be extracted from the data. This information is both elementspecific and local, the latter because of both the bond length disorder that increases with the distance from the absorber and dampens the contribution of higher order coordination shells, and the short photoelectron mean free path. ${ }^{110,111,121-131}$ Limited by this "nearsightedness", EXAFS provides "fast" snapshots of the local atomic and electronic environments: the photoexcited states have a very short life-time ( $\sim 1 \mathrm{fs})$, for which the absorption process is much shorter than the typical time scale of atomic vibrations (on the order of ps). Extracting this information from the experimental data is a multi-step process. First, using de Broglie's relationship between the wavenumber and momentum of the wave-particle, the wavenumber $k$ is expressed as: ${ }^{111}$

$$
k=\frac{1}{\hbar} \sqrt{2 m_{\mathrm{e}}\left(E-E_{0}\right)},
$$

where $m_{\mathrm{e}}$ is the electron mass, $\hbar$ is Planck's constant divided by $2 \pi, E_{0}$ is the core level ionization energy and $E$ is the energy of the incident X-rays. A smooth, isolated-atom background function $\mu_{0}(E)$ is subtracted from the raw, edge-step $\left(\Delta \mu_{0}\right)$ normalized, absorption coefficient $\mu(E)$ (Fig. 9a), isolating the EXAFS oscillations. Finally, we obtain the EXAFS signal as a function of $k$ (using eqn (20))

$$
\chi(k)=\frac{\mu(k)-\mu_{0}(k)}{\Delta \mu_{0}} .
$$

The resulting $\chi(k)$ pattern is generally weighted by a factor of $k^{n}(n=1-3)$ in order to amplify the weaker high- $k$ portion of the signal, as shown in Fig. 9b. Finally, the EXAFS pattern $\chi(k)$ is converted to real-space (r-space) by Fourier transformation, and the magnitude of the complex Fourier transform (FT magnitude) is presented, Fig. 9c. The first peak relates to the distribution of nearest neighbor distances to the absorber, e.g. Ce-O in the case of $\mathrm{CeO}_{2}$. Peaks at distances beyond those of the nearest neighbors are usually a combination of single- and multiplescattering contributions to EXAFS. In order to obtain structural information concerning nearest neighbors and bonding environments, the EXAFS data are fit using non-linear, multiple, least squares regression methods, that we describe below.

Eqn (22), representing the EXAFS spectrum, is a modern form of the original equation, derived by Stern, Sayers, and Lytle. ${ }^{121,126,127}$

$$
\chi(k)=S_{0}^{2} \sum_{i} N_{i} \frac{f_{i}(k)}{k r_{i}^{2}} \mathrm{e}^{-\frac{2 r_{i}}{\lambda(k)}} \mathrm{e}^{-2 k^{2} \sigma_{i}^{2}} \sin \left(2 k r_{i}+\delta(k)\right) .
$$

Each unique scattering path $i$ is characterized by $r_{i}$, defined as the distance between the absorbing atom and the $i$ th scattering atom (for single scattering) and, in general, is equal to half path-lengths for multiple-scattering paths. $f_{i}(k)$ is the "backscattering amplitude", proportional to the scattering cross section of the photoelectron. This function is element specific since heavier atoms (large $Z$ ) will scatter with greater probability than lighter atoms (small $Z$ ). For example, Ce atoms 

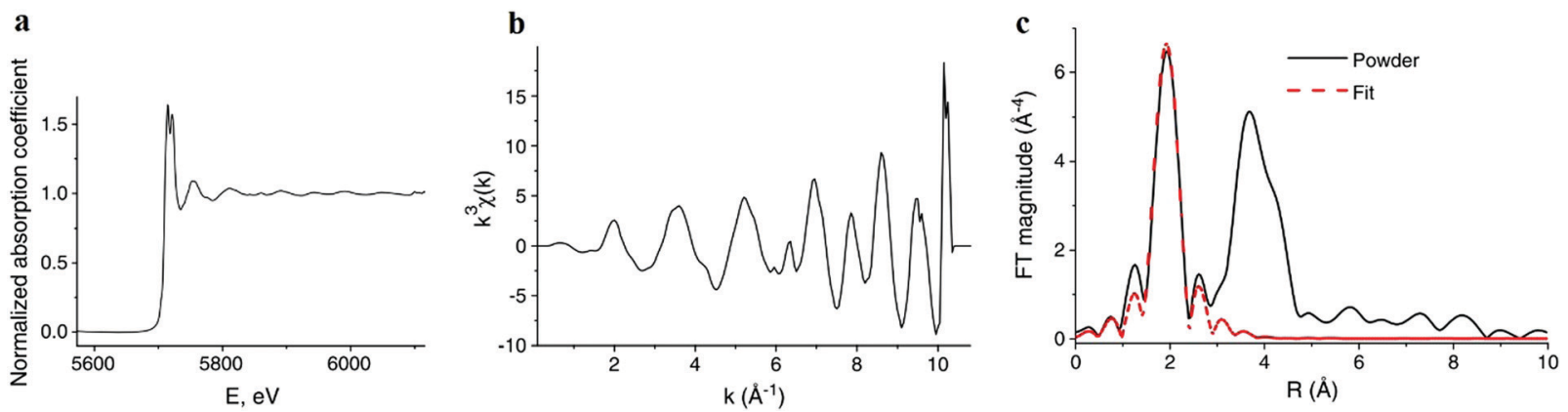

Fig. 9 (a) The normalized absorption spectrum at the $C e L_{\text {III }}$ edge for pure ceria powder. The normalization includes subtraction of a monotonically decreasing background function, causing the difference to Fig. 8 a and normalization of the edge step, defined as $\Delta \mu_{0}$. $\Delta \mu_{0}$ is the intensity difference between the average pre edge absorption (normalized to 0 ) to the post edge step absorption of the atom in the absence of neighbours (i.e. no white line or EXAFS features, normalized to 1). (b) The corresponding $k^{3}$-weighted EXAFS spectrum. (c) The corresponding Fourier transform magnitude of the data and theoretical fit for the first coordination shell of cerium. Adapted from ref. 45 with permission from Elsevier, copyright 2010.

scatter more strongly than the rather light oxygen atoms. Another term that depends on the identity of the scattering atom is the photoelectron phase shift $\delta(k)$ due to both the absorber and the scattering atom. The term $\frac{1}{k r_{l}^{2}}$ accounts for the spherical nature of the wavefunction. The wavefunctions of the final and initial states of the $(Z-1)$ electrons that are not excited by the $\mathrm{X}$-ray photon, do not completely overlap. To correct for this effect, which is not accounted for in the singleelectron approximation used to derive eqn (22), the "amplitude reduction factor" $S_{0}{ }^{2}$, is introduced. Since this factor, to a first approximation, is only sensitive to the type of the absorbing atom, but not the scattering atoms, it does not depend on $k$, an approximation which is universally used in data analyses. Equivalent single scattering paths are represented by a multiplication factor $N_{i}$ representing the coordination number describing the absorber-scatterer geometry. For example, in undoped ceria, all first shell oxygen neighbors of a $\mathrm{Ce}^{4+}$ cation in octahedral coordination $\left(N_{i}=8\right)$, will contribute approximately the same EXAFS signal. In this respect, one of the challenges in EXAFS analysis is accounting for "multiple scattering" effects that may make significant contributions in materials with high symmetry, such as those with fluorite structure. The difficulty arises because of the high degeneracy of such paths that interfere constructively in high symmetry structures. The mean free path term, $\mathrm{e}^{-\frac{2 r_{i}}{\lambda(k)}}$ appears due to inelastic scattering processes and the finite core-hole lifetime. This is one of the reasons why EXAFS is a local probe, only characterizing the environment $\leq 6-8 \AA$ from the absorber. A Debye-Waller type term, $\mathrm{e}^{-2 k^{2} \sigma_{i}^{2}}$, describes the suppression of $\chi(k)$ due to disorder, including both thermal (dynamic, i.e., vibrational) and static (configurational) disorder. The parameter $\sigma_{i}^{2}$ is defined as the variance in the interatomic half path length $r_{i}$, and is often abbreviated as "mean square radial displacement (MSRD)", or, alternatively, as the EXAFS DebyeWaller factor (DWF). It is important to note that the Gaussian approximation that gives rise to the Debye-Waller term in EXAFS eqn (22) is often not adequate when significant asymmetry of the pair distribution function is present, for example at elevated temperatures ${ }^{132}$ or in strongly anharmonic materials. ${ }^{133}$ In those cases, the contribution of the pair distribution function to eqn (22) can be analyzed via cumulant expansion as described by ref. 111 and in ref. 122 .

\subsection{Local structure of ceria solid solutions as characterized by EXAFS}

Being element specific, EXAFS is particularly useful for the study of doped ceria compounds $\mathrm{Ce}_{1-x} \mathrm{Do}_{x} \mathrm{O}_{2-x / 2}$ where $x$ is the nominal molar fraction of the extrinsic dopant on the cationic site, and $\mathrm{Do}^{3+}$ refers to the trivalent dopant atom. In these solid solutions, separately addressing the local environment around cerium and around the aliovalent dopant atoms is essential for understanding the macroscopic defect-related properties such as ionic conductivity, ${ }^{3,134}$ electrostriction and anelasticity. ${ }^{135}$ However, the absorption edges of different elements may be too close to each other so that their EXAFS spectra overlap, complicating analyses of both edges. Therefore, to take full advantage of the information contained in an EXAFS spectrum, the absorption edge $\left(\mathrm{L}_{\mathrm{I}}, \mathrm{L}_{\mathrm{II}}\right.$ or $\left.\mathrm{L}_{\mathrm{III}}\right)$ of Ce and Do must be chosen with care in order to separate them as much as possible. The Ce $\mathrm{L}_{\mathrm{I}}, \mathrm{L}_{\mathrm{II}}$ and $\mathrm{L}_{\mathrm{III}}$ edges occur at $\sim 6549, \sim 6164$, and $\sim 5724 \mathrm{eV}$, respectively. For example, in $\mathrm{Sm}^{3+}$-doped ceria, the $\mathrm{Sm} \mathrm{L}_{\mathrm{III}}$ edge located at $\sim 6719 \mathrm{eV}^{111}$ is close to the $\mathrm{Ce} \mathrm{L}_{\mathrm{I}}$, leading to overlap of EXAFS spectra originating from the low energy and high energy edges; therefore, when acquiring data for Sm-doped ceria, it is advantageous to probe the more isolated $\mathrm{Sm} \mathrm{L}_{\mathrm{II}}$ edge at $\sim 7315 \mathrm{eV}$ instead of the $\mathrm{Sm} \mathrm{L}_{\mathrm{III}}$ edge. ${ }^{136}$ In the following sections we review the short-range structural information reported in the literature for ceria solid solutions on the basis of the EXAFS investigations. It is important to note that major discrepancies are reported, even for same dopant cations and concentrations, which we discuss below. To begin explaining these differences, the following factors must be taken into account:

(i) The influence of dopant concentration on the short-range structure may be convoluted with microstructural effects because of the role of dopant level on microstructure evolution. For example, a change in doping concentration affects the average grain size through solute drag effects, and consequently 
the degree of defect segregation and local cation-anion distribution over grain and grain boundaries even for constant processing conditions. ${ }^{33,136}$

(ii) For equal doping levels, the synthesis route and conditions (e.g., Pechini, solid state or combustion synthesis, etc., temperature, atmosphere) can affect the local density and distribution of dopants in the structure. In turn, these will affect interatomic distances.

(iii) Extracting material parameters from EXAFS data involves multiple stages of data processing, as described in the previous section. Therefore, the fitting process is model dependent.

In summary, in the case of ceria compounds, due to the variety of factors affecting the local structure, it is preferred to focus on the relative trends in the EXAFS derived results rather than absolute values for a specific sample or composition.

3.2.1 Probing interatomic distances in aliovalent-doped ceria. EXAFS enables separate examination of the environments of Ce and dopant cations in the solid solutions; of the various parameters, particularly useful are the interatomic distances. Fig. 10 illustrates a doped ceria unit cell. Consistent with computational predictions, the vacancy is taken to reside in the first nearest neighbor shell of the dopant ion. The interatomic distances of relevance are the first and second coordination shells of the Ce atom, labeled (a) Ce-O and (b) Ce-Ce,

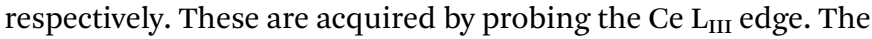
first and second coordination shells of the aliovalent dopant atom, are labeled (c) Do-O and (d) Do-Ce, respectively, and these are acquired by probing the Do edge. The first coordination shell radii around the oxygen-vacancy point-defect Cat- $\mathrm{V}_{\mathrm{O}}$ (where "Cat" is short for cation and represents either Ce or Do) cannot be directly acquired and are labeled (e and f).

As mentioned in the previous section, EXAFS data was collected over the years from ceria materials of different morphologies and preparation methods, however similar trends

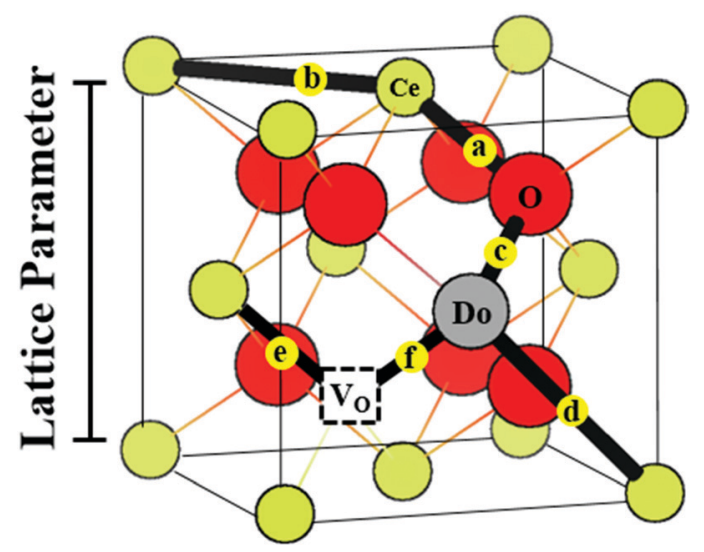

Fig. 10 Fluorite unit cell of a doped ceria solid solution, including $\mathrm{Ce}^{4+}$ and $\mathrm{O}^{2-}$ ions, and a single $\mathrm{Do}^{3+}$ (trivalent dopant) cation and one $\mathrm{V}^{\bullet \bullet}$ (oxygen-vacancy) as lattice defects. The cell illustrates the different interatomic distances commonly extracted from EXAFS analysis, and does not represent realistic stoichiometry or defect segregation. The interatomic distances are labeled (a). Ce-O; (b) $\mathrm{Ce}-\mathrm{Ce}$; (c) Do-O; (d) Do-Ce, while (e and f) represent the unknown distances around the vacancy defect. in EXAFS derived data appear for example for both bulk materials (powders and pellets) and sputter deposited thin films. Thin films differ from bulk ceramics by grain size, shape and orientation, but most notably by the existence of possible anisotropic strain. In such a case the film experiences either compressive (or tensile) strain in parallel to the substrate surface, and the opposite strain perpendicular to it and caused by the Poisson ratio. This type of anisotropic strain can reach up to $0.5 \%$ and is retained either intrinsically from the deposition process or as a result of heterogeneous layer stacking, ${ }^{27}$ or opposed stress patterns in free standing film membranes. ${ }^{137}$ It is important to stress that this anisotropy is relatively small and does not necessarily deem the unit cell to distort to lower symmetry. This effect can be readily detected and quantified by examining the in- and out-of-plane lattice parameters using $\mathrm{XRD}$, e.g. described in ref. 138. In addition it can be qualitatively detected using Raman spectroscopy, as measured by Rupp and co-workers e.g. see ref. 137. The effect of anisotropic strain on the Raman spectra of doped and undoped ceria thin films is discussed intensively in Section 4.3. The impact of anisotropic strain on the interatomic distances measured by EXAFS was investigated by Kossoy et al. ${ }^{59}$ for $20 \mathrm{~mol} \% \mathrm{Gd}$ doped, 200-450 nm thick films. Fig. 11 illustrates that while films with no anisotropic strain (strain free) produce first and second shell interatomic distances similar to the equivalent powder control, the presence of anisotropic strain slightly reduces the measured interatomic distances most significantly for the most abundant $\mathrm{Ce}-\mathrm{O}$ interatomic distance. This effect has to be kept in mind when comparing thin films to bulk
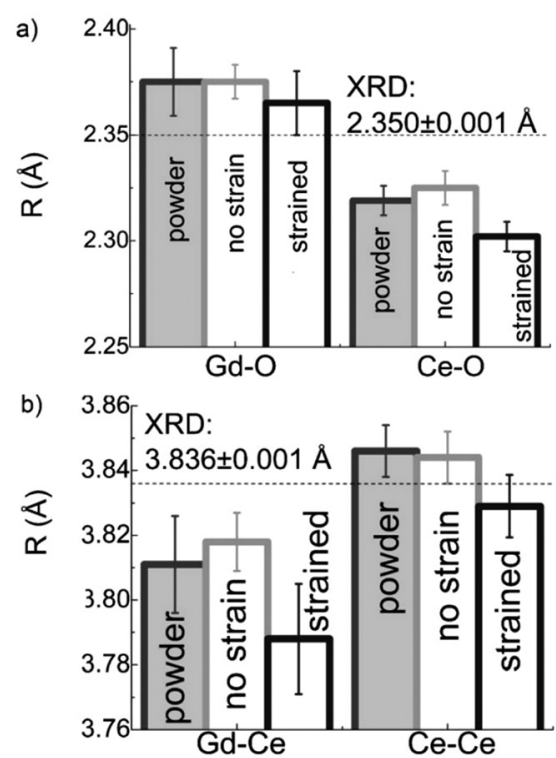

Fig. 11 First shell (a) and second shell (b) interatomic distances, as measured by EXAFS for $\mathrm{Ce}$ and $\mathrm{Gd}$ absorption edges of $20 \mathrm{~mol} \% \mathrm{Gd}$ doped ceria materials. The investigated materials are in the form of powders ( $>1 \mu \mathrm{m}$ grain size) and films deposited by magnetron sputtering with and without compressive in plane strain. The equivalent interatomic distance as derived from an XRD measurement of the lattice parameter is given by a dashed line in each panel. Reproduced from ref. 59, with permission from John Wiley \& Sons, Inc., copyright 2010. 
pellets and powders in the following sections. The decrease in $\mathrm{Ce}-\mathrm{O}$ distances due to a lower intrinsic compaction of a film with the presence of anisotropic strain was interpreted as a form of stress relaxation in the Ce first shell environment mediated by nearby oxygen vacancies.

In addition to capturing the impact of anisotropic strain on local structure, Fig. 11 also introduces the major differences observed using EXAFS around the host cation (Ce) and the dopant cation (Gd) first and second shell environments. The first shell distance Cat-O is greater around Gd as expected from the dopant's larger ionic radius compared to $\mathrm{Ce}^{4+}$, however, counter intuitively the second shell distance is greater around $\mathrm{Ce}^{4+}$. To compare this effect for several doped ceria materials, Fig. 12 summarizes representative first shell $\mathrm{Ce}-\mathrm{O}$, Do-O and second shell $\mathrm{Ce}-\mathrm{Ce}$, Do-Ce distances obtained by EXAFS studies as functions of Shannon ionic radii for octahedral coordination $(N=8) \cdot{ }^{139} \mathrm{Yb}$, Er and Sm-doped ceria data are taken from Giannici et al., ${ }^{136}$ whereas Y, Gd and La-doped ceria data are taken from Deguchi et al. ${ }^{107}$ These reports were selected since they include both first and second coordination shells for two doping fractions of 10 and $20 \mathrm{~mol} \%$ dopant in ceria, thus allowing systematic consideration of the role of the dopant type on the interatomic distances illustrated in Fig. 10, as well as the influence of concentration.

Considering first the Cat-O distances for small dopants ( $\mathrm{Yb}, \mathrm{Er}$ and $\mathrm{Y})$ the $\mathrm{Ce}-\mathrm{O}$ and Do-O distances are similar. For dopants with radius above $105 \mathrm{pm}$ (Gd, Sm, La), the Do-O distance is in fact larger than the $\mathrm{Ce}-\mathrm{O}$ distance and this discrepancy increases with dopant ionic radius. This result is supported by other reports, ${ }^{106,116,140-142}$ and it is intuitively expected for dopants with larger ionic radius to have larger distance to neighboring oxygen ions. However, even though ionic radii of $\mathrm{Yb}^{3+}, \mathrm{Er}^{3+}$ and $\mathrm{Y}^{3+}$ are larger than $\mathrm{Ce}^{4+}$, their first shell radii are very similar to that of $\mathrm{Ce}$, for both 10 and $20 \mathrm{~mol} \%$ doping. The intuitive interatomic distance relationships arrived at by comparing ionic radii of $\mathrm{Ce}^{4+}$ and $\mathrm{Do}^{3+}$ also do not apply to the second coordination shell. In the bottom panels, describing the second coordination shells around Ce and dopants for 10 and $20 \mathrm{~mol} \%$ doping, we observe that firstly, Ce-Ce distances are slightly larger than Do-Ce distances, even for the largest trivalent dopants reported for the series i.e., Sm and La. Secondly the second shell environments of Ce and Do are much more similar to each other than the first shell environments. These second shell effects, were also noted by Wang et $a l .{ }^{105}$ for $\mathrm{Y}$ and Kossoy et al. for $20 \mathrm{~mol} \% \mathrm{Gd}$-doped powders, ${ }^{116}$ as well as for sputter deposited films with and without compressive in-plane strain as described in Fig. $11{ }^{59} \mathrm{It}$ can be concluded by EXAFS analysis on trivalent-cation doped ceria solid solutions that: (i) for dopants with $R_{\text {Shannon }} \geq$ 105 pm, Ce-O < Do-O while Ce-Ce > Do-Ce. This discrepancy suggests that the lattice is strongly distorted in the vicinity of dopant ions and fluorite symmetry is not preserved on the local level, even when the dopant cation concentration is as little as $10 \mathrm{~mol} \%$, where no long-range ordering of defects is observed. ${ }^{33,35}$ (ii) The smaller discrepancy between the Do and Ce second shells as compared to the first shells, reveals that local lattice distortions, if present, are much more significant in the first shell than the second. (iii) These trends with ionic radius are generally insensitive to dopant concentration in the range $10-20 \%$. A focused evaluation of the role of concentration is presented below.

3.2.2 Averaged XRD structure vs. local element specific structure obtained by EXAFS. The combination of EXAFS and $\mathrm{X}$-ray diffraction (XRD) is particularly useful to construct a comprehensive multi-scale picture of a material, since XRD is able to produce averaged, non-element specific information with very high accuracy, while EXAFS acts as an element-specific probe of the local environment, providing information on lattice distortions in the vicinity of randomly distributed point-defects to which XRD is insensitive. Until recently, due to the limited reports of EXAFS results, the crystallographic literature on ceria has been dominated by conventional XRD, which, because of the absence of long-range ordering upon doping, reports the globally averaged fluorite crystal structure. ${ }^{33,35,60,143-146}$ Thus, many reviews describe moderately doped ceria $(\leq 20 \mathrm{~mol} \%)$ as a single fluorite phase. The differences described in the previous section between $\mathrm{Ce}-\mathrm{O}$ and Do-O distances immediately imply that average interatomic distances obtained from XRD methods will disagree with the analogous distances derived by EXAFS. ${ }^{132,147-151}$ In the following sections, we compare the interatomic distances implied by these two methods for ceria solid solutions. The results that emerge from these comparisons are indicative of the presence of local distortions in ceria solid solutions, for which possible models are discussed.

3.2.3 The interatomic distances in doped ceria: EXAFS vs. XRD. To compare EXAFS and XRD data, it is preferable to do so for the same set of samples. However, rather few papers, discussing EXAFS of ceria solutions, provide the complimentary XRD data. As an example, we consider a preliminary comparison of the two data sets presented by Kossoy et al. for $20 \mathrm{~mol} \%$ Gd-doped ceria solutions. ${ }^{59}$ Under the assumption that the FCC lattice symmetry of the fluorite structure is preserved, the first shell interatomic distance between cation-anion pairs $R_{\mathrm{XRD}}^{\mathrm{Cat}-\mathrm{An}}$ depends on the lattice parameter $a$, by:

$$
R_{\mathrm{XRD}}^{\mathrm{Cat}-\mathrm{An}}=\frac{\sqrt{3}}{4} \cdot a .
$$

If symmetry is also preserved around all cation sites, a simple weighted average of the constituent individual distances, the real $R_{\mathrm{EXAFS}}^{\mathrm{Cat}-\mathrm{O}}$ and that representative of vacancy sites, $R_{\mathrm{Calculated}}^{\mathrm{Cat}-\mathrm{V}_{\mathrm{O}}}$, produces an equation relating XRD and EXAFS results:

$$
R_{\mathrm{XRD}}^{\mathrm{Cat}-\mathrm{An}}=\left(1-\frac{x}{4}\right) \cdot R_{\mathrm{EXAFS}}^{\mathrm{Cat}-\mathrm{O}}+\frac{x}{4} \cdot R_{\text {Calculated }}^{\mathrm{Cat}-\mathrm{V}_{\mathrm{O}}}
$$

Here, $R_{\text {EXAFS }}^{\text {Cat-O }}$ is the weighted average of the EXAFS measured first shell cation distances and $x$ is the molar fraction of the dopant, given as:

$$
R_{\mathrm{EXAFS}}^{\mathrm{Cat}-\mathrm{O}}=R_{\mathrm{EXAFS}}^{\mathrm{Ce} \cdot \mathrm{O}} \cdot(1-x)+R_{\mathrm{EXAFS}}^{\mathrm{DO}-\mathrm{O}} \cdot x
$$

Combining eqn (24) and (25) yields an expression for the Cat $-\mathrm{V}_{\mathrm{O}}$ distance (which cannot be directly measured), given by

$$
R_{\text {Calculated }}^{\mathrm{Cat}-\mathrm{V}_{\mathrm{O}}}=\frac{4}{x} \cdot R_{\mathrm{XRD}}^{\mathrm{Cat}-\mathrm{An}}-\left(\frac{4}{x}-1\right) \cdot R_{\mathrm{EXAFS}}^{\mathrm{Cat}-\mathrm{O}} .
$$


a

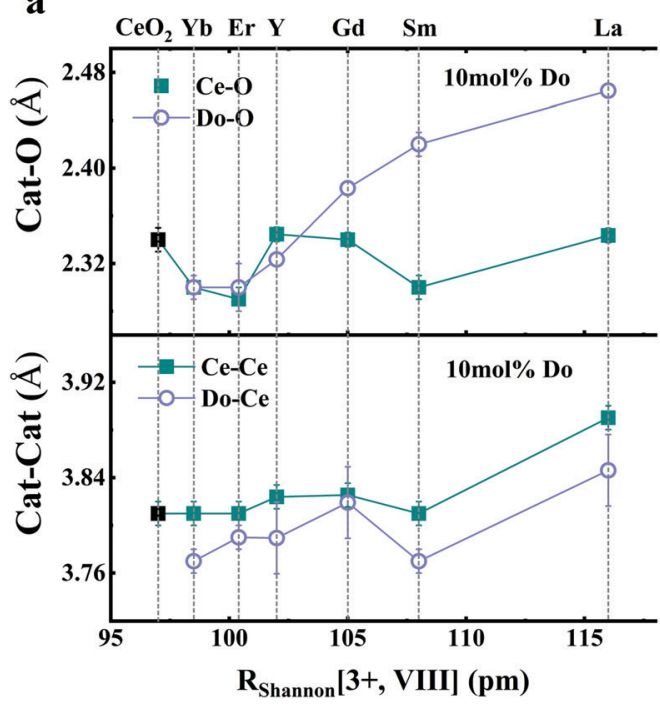

b

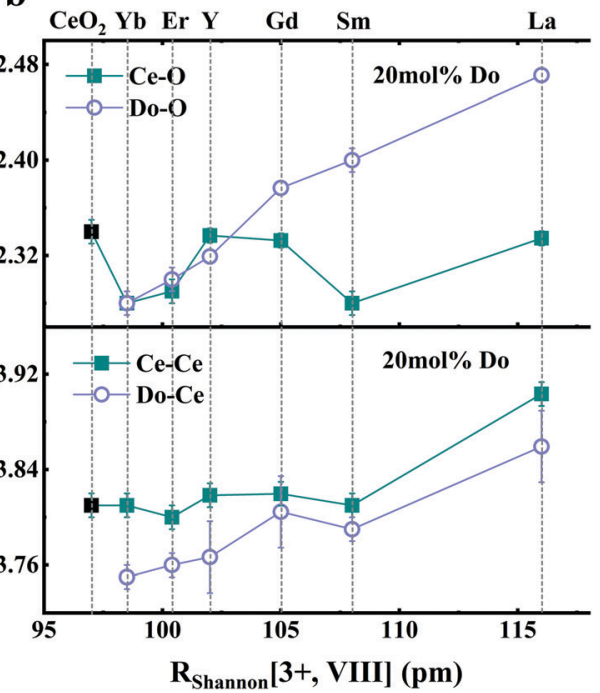

Fig. 12 Top panels give the first coordination shell distances for $\mathrm{Ce}-\mathrm{O}$ (squares) and for dopant atoms Do-O (circles) in ceria solid solutions with $10 \mathrm{~mol} \%$ (a) and $20 \mathrm{~mol} \%$ (b) dopant fraction, as a function of Shannon ionic radius $R_{\text {Shannon. }}$. Bottom panels present the corresponding second coordination shell distances. The top axis indicates the type of doping and the bottom axis shows the ionic radius for octahedral coordination. Y, Er and Sm-doped ceria solutions were prepared using solution combustion synthesis, calcined, sintered and pressed into pellets as described in ref. 136. Y, Gd and La-doped ceria were prepared using a solid state reaction method, calcined sintered and pressed into pellets as described in ref. 107. Materials in both data sets have micro-crystalline grain size. The data used for this figure are summarized in Table S1 of the ESI. $\dagger$

An analysis of literature data, which provide both EXAFS and XRD results, is summarized for Gd and Sm dopants in Fig. 13.

Inaba et al. ${ }^{154}$ suggested that the cations in the first coordination shell of the vacancy site move away from it due to electrostatic repulsion. Marrocchelli et al. ${ }^{153}$ estimated the repulsion of $\mathrm{Ce}^{4+}$ away from the vacancy site in reduced ceria, based on molecular dynamics (MD) calculations, assuming the preservation of fluorite symmetry and using known ionic radii. The result was a shift of the $\mathrm{Ce}^{4+}$ ions away from the vacancy site by $\sim 0.1 \AA$. With respect to Ce-O distance of $2.34 \AA$ as calculated from EXAFS for $\mathrm{CeO}_{2}$ (see Fig. 12), the MD based calculation brings the predicts therefore a Cat $-\mathrm{V}_{\mathrm{O}}$ distance of $\sim 2.45 \AA$ marked by a dashed line in Fig. 13. Calculation of the Cat $-\mathrm{V}_{\mathrm{O}}$ distance by eqn (26) produces large error bars at low dopant concentrations due to the appearance of the doping fraction in the denominator. It is none the less clear that available XRD and EXAFS values are for the most part (excluding Nitani et al. data ${ }^{106}$ ) unable to produce reasonable Cat $-\mathrm{V}_{\mathrm{O}}$ distances using the above assumptions, with some values being as large as the second coordination shell radii, at $\sim 3.8 \AA$. Importantly, these unphysical values are calculated under the incorrect assumption that the lattice structure of ceria is perfectly preserved in the first coordination shell of defects.

The second cation coordination shell (Cat-Cat) radii can be compared for EXAFS and XRD derived data in a similar fashion and are shown to produce no detectable discrepancy. ${ }^{45}$ This indicates again that a distortion in the fluorite symmetry is more significant in the cations' first coordination shells for undoped and doped ceria structures. Recently, Shirbhate et al. ${ }^{155,156}$ studied more complex ceria systems with mixed dopants, $\mathrm{Ce}_{0.85}(\mathrm{Sm})_{0.075}(\mathrm{M})_{0.075} \mathrm{O}_{2-\delta}($ where $\mathrm{M}=\mathrm{Sm}, \mathrm{Sr}, \mathrm{Gd}, \mathrm{Nd}$,

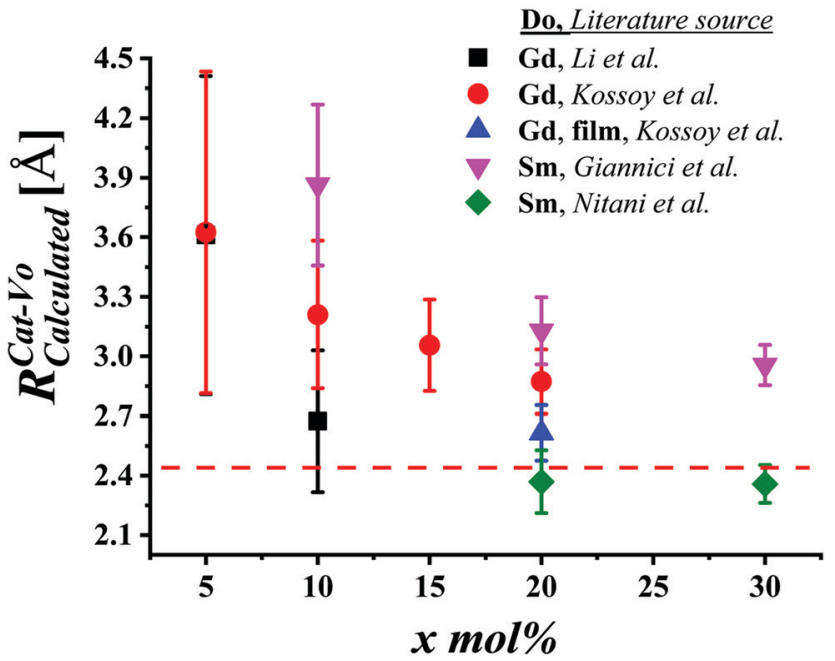

Fig. 13 Cat- $V_{O}$ distances $R_{\text {Calculated }}^{\text {Cat- } V_{O}}$, derived using eqn (26) from combined XRD and EXAFS data, assuming preservation of fluorite $F m \overline{3} m$ symmetry, as a function of dopant mol\% $(x)$. The Gd-doped ceria data are taken from Li et al. ${ }^{152}$ and Kossoy et al. ${ }^{116}$ for powders, from Kossoy et al. ${ }^{59}$ for Gd-doped ceria films, and for Sm-doped powders from Giannici et al. and Nitani et al. ${ }^{106,136}$ The data for the figure are summarized in Table S2 of the ESI. $\dagger$ The dashed line is the numerically computed Cat- $V_{O}$ distance $\sim 2.45 \AA$ based on the molecular dynamics (MD) estimate from Marroccelli et al. ${ }^{153}$

$\mathrm{Ca}$, and Dy), using Rietveld analysis and EXAFS. Here too, local and average first shell distances, were found to be difficult to reconcile. Very low values for the EXAFS Ce-O distances were obtained, compared to values derived from XRD and Rietveld analysis using a fixed fluorite unit cell, again, suggestive of lattice distortions not captured in the XRD results. 
3.2.4 Changes in lattice structure as a function of dopant concentration: EXAFS vs. XRD. When comparing only two doping concentrations for ceria -10 and $20 \mathrm{~mol} \%$, for a given dopant (Fig. 12), we cannot discern a significant difference between the two sets of interatomic distances. This is owed to the fact that the uncertainties in EXAFS derived Cat-O distances in the EXAFS literature range from 0.2 to $0.4 \%$ of the average bond length, while changing dopant concentration (usually in 5 or $10 \mathrm{~mol} \%$ increments) or changing dopant ionic radius, introduce about $0.1 \%$ change in the lattice parameter. The effect detected in Ce-O or Do-O distances as a result, is not very strong. For comparison, XRD uncertainties are only about $0.002 \%$ for the lattice parameter, $a$, so lattice parameter changes introduced by doping can be precisely measured. Trends in the Ce-O and Do-O distances with dopant concentration and dopant ionic radius, however, do exist and can be isolated as described below. It is important to note that these trends have become a major topic of discussion, primarily due to their seeming incompatibility with XRD derived distances as a function of dopant concentration, as we elaborate in the following.

It has long been known that for dopants larger than $\sim 102 \mathrm{pm}$, such as $\mathrm{Gd}^{3+}$ and $\mathrm{Sm}^{3+}$, the average lattice parameter in the fluorite phase, as obtained using X-ray diffraction methods, increases with doping concentration. ${ }^{157,158}$ However, several studies conducted on doped ceria using local probes, such as EXAFS, ${ }^{58,107,116,136,140-142}$ have found that cation-oxygen distances Cat-O, labeled a and $\mathrm{c}$ in Fig. 10, do not follow the average lattice parameter and contract as a function of increasing dopant concentration. These results were also supported by X-ray and neutron scattering based PDF measurements. ${ }^{144-146}$ Ohashi et al. were the first to note this discrepancy for Gd-doped ceria. Shortly thereafter, Yamazaki et al. acquired XAFS data for doped ceria for multiple dopants at several concentrations and reported the Ce-O distance trend presented in Fig. $14 .{ }^{140}$ According to more recent works, ${ }^{116,136,142}$ this average reduction in interatomic distances with increasing dopant fraction is accompanied by increasing disorder as indicated by an increase in the $\sigma^{2}$ factor (mean square radial displacement), as given in eqn (22).

Building on the above observations, we have collected here all available EXAFS data of the type shown in Fig. 14 for various dopants, all approximately within the fluorite range (dopant fraction, $x \leq 0.3$ ). In addition, we have collected complementary XRD lattice parameter data, and derived $R_{\mathrm{XRD}}^{\mathrm{Cat}-\mathrm{O}}$ using eqn (23). To a first approximation, the interatomic distances vary linearly with dopant concentration, as exemplified in Fig. 14, prompting us to compute the slopes, $\Delta[$ Cat-An $] / \Delta[x]$, as a metric for capturing the differences between the local (EXAFS) and average (XRD) trends. We applied linear regression analysis to each data set and extracted the approximate slope in units of $\AA$ per mol\% dopant. The complete dataset and slopes are provided in the ESI, $\dagger$ Tables S3-S12. The results are presented as a function of the trivalent dopant Shannon ionic radius for 8-coordination ${ }^{139}$ in Fig. 15. The error bars reflect both the uncertainties in the original data and the nonlinearities in the distance $v s$. dopant fraction plots ${ }^{33}$ (which is particularly pronounced in the case of

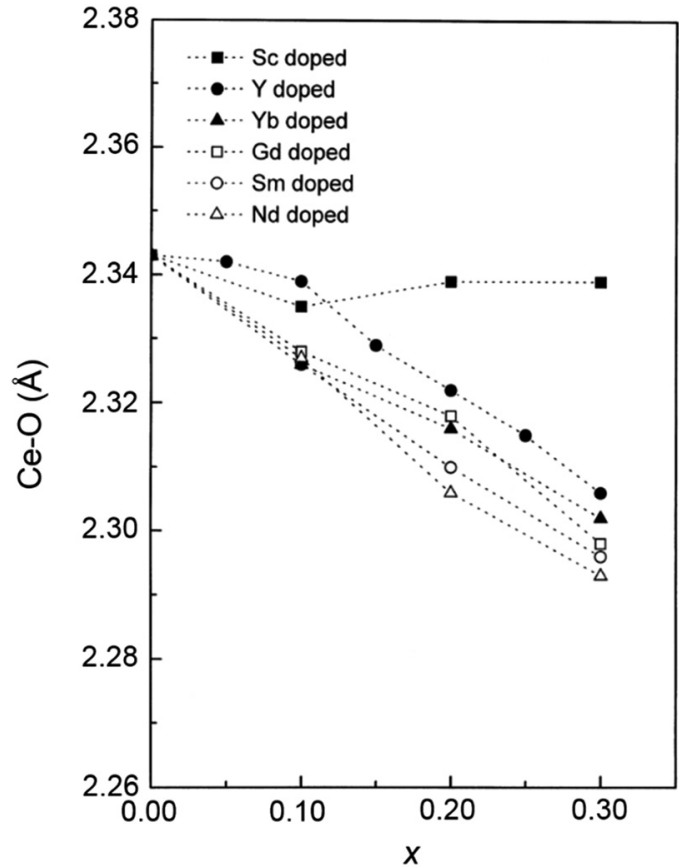

Fig. $14 \mathrm{Ce}-\mathrm{O}$ interatomic distances in ceria doped with Sc, Y, Yb, Gd, Sm and $\mathrm{Nd}$ as a function of nominal dopant fraction $x$. Adapted from ref. 140 with permission from Elsevier, copyright 2000.

the XRD data of Nd-doped ceria ${ }^{94}$ ). The linear fits are intended to demonstrate qualitatively the relative contraction/expansion of the lattice parameter and of the different local environments defined by the dopant choice and concentration in the ceria solid solution.

The trend from Cat-An data measured by standard XRD (gray triangles) is well described in the literature and qualitatively follows Vegard's law. It clearly shows the linear dependence of lattice expansion (positive values on the vertical axis) and contraction (negative values on the vertical axis) with respect to the dopant ionic radius. Note that contrary to expectation, zero contraction does not coincide with a dopant of ionic radius near $\mathrm{Ce}^{4+}$ (hollow red circle at $97 \mathrm{pm}$ ). Intuitively, we would expect the lattice parameter to increase upon addition of a substitutional dopant larger than the host. However, it has been known for some time on the basis of experimental data ${ }^{157}$ that the critical dopant radius at which expansion/contraction with respect to dopant fraction is at minimum is given by a value of $\sim 102 \mathrm{pm}$ for ceria. Molecular dynamics (MD) and density functional theory (DFT) simulations later produced a slightly larger critical dopant radius of $105 \mathrm{pm}$ (Gd). ${ }^{153}$ Indeed, zero expansion in Fig. 15: is observed in the 102-105 pm range for Dy (102.7 pm) and $\mathrm{Tb}(104 \mathrm{pm})$ from literature values of up to $x \leq 0.23$ and $x \leq 0.18$ respectively (see Table S12 in the ESI $\dagger$ ). This effect was attributed to an overall contraction in the second nearest neighbor shell about the vacancy, composed of oxygen atoms, moving inwards to compensate for the partial positive charge. ${ }^{153,154}$ However, referring back to Fig. 12, no expansion is observed in $\mathrm{Yb}-\mathrm{O}$, Er-O and $\mathrm{Y}-\mathrm{O}$ distances compared to $\mathrm{Ce}-\mathrm{O}$ (although all dopants are slightly larger than $\mathrm{Ce}$ ), 
suggesting that contraction in the lattice parameter in these cases does not necessarily originate only from the contraction of oxygen atoms around the vacancy site but is inherent to the dopant environment itself.

Turning to the EXAFS data, for all dopants with $R_{\text {Shannon }}>$ $97 \mathrm{pm}$, contraction of $\mathrm{Ce}-\mathrm{O}$ upon doping (green squares) is observed. The contraction magnitude of $\mathrm{Ce}-\mathrm{O}$ increases in the dopant radius range between $\mathrm{Er}$ and $\mathrm{Nd}$. $\mathrm{Sc}$ and $\mathrm{Yb}$ (having generally a strong tendency to form Do- $\mathrm{V}_{\mathrm{O}}$ clusters, i.e. low association energy, [see Fig. 7 and ref. 161]) and La (having the highest association energy of dopants discussed here) lie outside the trend. The behavior of Do-O (purple circles) follows closely the Ce-O trend (green squares), with the exception of La, which is the only cation that reveals expansion of the Do-O distance upon doping. Sc is the only dopant with ionic radius $<97 \mathrm{pm}\left(\mathrm{Ce}^{4+}\right)$ for which EXAFS data are available. It exhibits a significant contraction in lattice parameter, yet no recorded change with $x$ in the local $\mathrm{Ce}-\mathrm{O}$ distance. As observed by Yamazaki et al. ${ }^{140}$ using XRD, Sc tends to phase separate as $\mathrm{Sc}_{2} \mathrm{O}_{3}$ even for $x<0.3$. This may suggest that fewer dopants (and vacancies) are available in the vicinity of Ce which ordinarily contribute to $\mathrm{Ce}-\mathrm{O}$ bond contraction (N.B. - there are at present no EXAFS data which include $\mathrm{Sc}-\mathrm{O}$ distances). It is apparent that much experimental, local structural data are missing, particularly for Do-O distances, and for dopants smaller than $\mathrm{Ce}^{4+}$. Obtaining such data with high accuracy can help focus and assist in a discussion of ceria solid solution local structure.

3.2.5 Modelling of the nearest neighbor shell distance as a function of dopant fraction. Several models have been proposed to explain why nearest neighbor $(1 \mathrm{NN})$ shell distances for Ce and Do are a decreasing function of $x$ for dopants which produce an increase in the lattice parameter and what controls the extent of contraction. Shortly after Ohashi et al. ${ }^{141}$ published the Cat-O EXAFS results for $\mathrm{Gd}$, Inaba et al. $^{154}$ suggested, based on molecular dynamics simulations, that shorter bond lengths result from contraction of the 6 oxygen atoms in the next nearest neighbor $(2 \mathrm{NN})$ shell of the vacancy. Andersson et al. ${ }^{162}$ later made additional $a b$ initio calculations in support of this model. However, the model predicted larger dopants should yield smaller $\Delta[$ Cat $-\mathrm{An}] / \Delta[x]$, which is partly correct for La, but is not consistent with the increasing contraction observed along the $\mathrm{Er} \rightarrow \mathrm{Nd}$ line in Fig. 15. Yamazaki et al. ${ }^{140}$ and later Giannici et $a .^{136}$ used an ion-packing model which preserved local fluorite symmetry, to discuss EXAFS local Cat-O trends with $x$ in ceria solid solutions. The model relied on a decrease in Shannon ionic radii upon reduction in coordination number, as a result of defect clustering (i.e. decrease in association energy near a certain species with increasing $x$ ). Indeed, it was able to qualitatively produce $\mathrm{Ce}-\mathrm{O}$ and $\mathrm{Do}-\mathrm{O}$ reduction with $x$. However, the authors noted that although local trends can be qualitatively predicted, the ion packing model has several major inconsistencies. In order to satisfy the experimental decrease in $\mathrm{Ce}-\mathrm{O}$ distances using the ion packing model, the ionic radii need to be brought down by $1-4 \%$. In addition, the model could not explain the EXAFS results

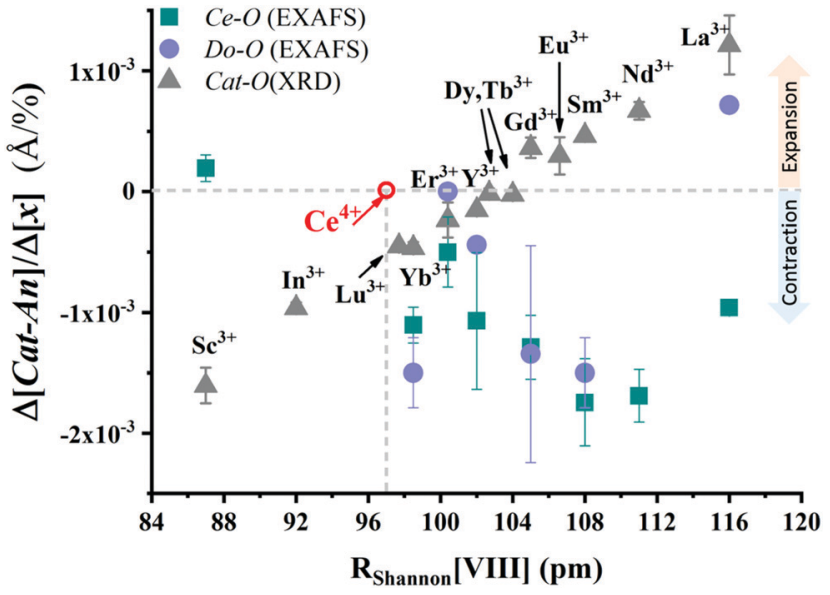

Fig. 15 The vertical axis gives values of the slopes extracted from Cat-An distances $(\AA)$ as a function of dopant fraction $x$ in mol\%, for the assumed fluorite lattice phase $(x \leq 0.3)$, using linear regression for EXAFS and XRD analysis. Green squares show the values of slopes extracted from first coordination shell distances of Ce measured by EXAFS. Purple circles show the values of slopes extracted from first coordination shell distances of the dopant, measured by EXAFS for the same material. Gray triangles show values of slopes extracted from average Cat-An distances calculated from XRD by using eqn (23). We used XRD and EXAFS data from the same report when available, including XRD data from additional sources: Yb, 136,140,157 $\mathrm{Er}^{136,157} \mathrm{Sm},{ }^{136,140,146,157} \mathrm{Gd} .{ }^{107,116,140,141,159}$ For dopants where only EXAFS data were available, we collected XRD data from external sources: $\mathrm{Sc}^{140,152} \mathrm{Y}^{107,140,145} \mathrm{Nd},{ }^{94,140,157} \mathrm{La}{ }^{61,107,157}$ In addition XRD data were added for dopants where EXAFS local data were not available: In, ${ }^{152}$ Lu, $^{160}$ $\mathrm{Dy},{ }^{157} \mathrm{~Tb}, \mathrm{Eu},{ }^{61}$ in order to facilitate discussion. Where multiple EXAFS and XRD data sets were available, we averaged the trends. The materials in ref. 145, 146 and 152 have nanocrystalline grains while the data used from other reports includes materials with microcrystalline grains. Materials in refs: Deguchi et al., ${ }^{107}$ Varenik et al., ${ }^{160}$ Yavo et al., ${ }^{159}$ Hong et al. ${ }^{157}$ and ref. 136 were measured as pellets, other data represents powder samples. The data used to construct this figure are summarized in ESI, $\dagger$ Tables S3 and S12.

presented in Fig. 12, where Ce-Ce distances are larger than Sm-Ce distances. A similar conclusion to that of Section 3.2.3 has been drawn by Giannici et al. that in order to properly describe local structure and the structural dependence on dopant content, we must consider some form of local distortion in the crystal. Recently, Koettgen et al. ${ }^{142}$ measured Ce-O distances using EXAFS for a series of Sm-doped ceria solutions in the range $x \leq 0.3$. The decreasing Ce-O distances (with $x$ ) were used in combination with known Shannon ionic radii for $N=8$ and $N=6$ to derive a decreasing (with $x$ ) set of coordination numbers. A comparison to coordination numbers derived using XRD and prior simulations data ${ }^{163}$ suggested that the vacancy distribution in Sm-doped ceria is close to random, namely there is no preference for $\mathrm{Sm}-\mathrm{V}_{\mathrm{O}}$ over $\mathrm{Ce}-\mathrm{V}_{\mathrm{O}}$ association. In summary, the models proposed above link the decrease in average coordination number around Ce or Do with $x$ to the decrease in Cat-O distances. This correlation qualitatively agrees with the data summarized in Fig. 15: for example, in the case of Sm, where defect association is expected to be random, the contraction in $\mathrm{Ce}-\mathrm{O}$ and $\mathrm{Do}-\mathrm{O}$ bond lengths are comparable, while for $\mathrm{La}$, where $\mathrm{Ce}-\mathrm{V}_{\mathrm{O}}$ associates are expected 
to be more frequent then $\mathrm{La}-\mathrm{V}_{\mathrm{O}}$ associates (due to high association energy) Ce-O contraction is more significant then La-O contraction. However, previously mentioned factors such as: (i) the counterintuitive result for second shell distances where Ce-Ce > Do-Ce (Fig. 12) as well as, (ii) the inability to produce physically acceptable $\mathrm{Ce}-\mathrm{V}_{\mathrm{O}}$ distances from combined XRD and EXAFS data, assuming fluorite structure preservation (Fig. 13), suggest that the proposed models should be expanded in order to accommodate local deviation from fluorite symmetry. Evidence for such local structural changes is discussed below.

3.2.6 First and second cation coordination shell environments: moving from fluorite to double-fluorite structure? Kossoy et al. $^{116}$ used EXAFS to monitor $\mathrm{Ce}-\mathrm{O}$ and $\mathrm{Gd}-\mathrm{O}$ bond lengths across the full range of solid solutions between the end members, $\mathrm{CeO}_{2}$ (fluorite structure) to $\mathrm{Gd}_{2} \mathrm{O}_{3}$ (double-fluorite structure), see Fig. 16. The authors found a sharp decrease in the $\mathrm{Ce}-\mathrm{O}$ distance at the onset of the transition from fluorite to double-fluorite as detected by XRD (between $0.2<x<0.25$ ). ${ }^{60}$ In contrast, the $\mathrm{Gd}-\mathrm{O}$ distance undergoes a monotonic decrease towards the average value in $\mathrm{Gd}_{2} \mathrm{O}_{3}$. Significantly, this full range EXAFS scan illustrated that the local Cat-O bond contraction in the fluorite range (up to $30 \mathrm{~mol} \% \mathrm{Gd}$ ) shown in Fig. 15 is part of a continuous process occurring throughout the full doping range. In a recently published review, Artini $^{33}$ demonstrated that the dopant fraction at which the double-fluorite phase is detected by XRD varies between 20 and $60 \mathrm{~mol} \%$, depending on the dopant. In addition, Artini et al. discuss evidence from Raman spectroscopy ${ }^{35,60}$ and high-resolution TEM, ${ }^{164}$ supporting the presence of nanometer-sized, spatially uncorrelated (i.e., no long-range order) regions, which fully transformed to the doublefluorite structure, interspersed within the predominantly fluorite phase. Also recent DFT calculations by Zguns et al. predict oxygen vacancy ordering in a C-type structure, even when Gd ions are randomly distributed. ${ }^{165,166}$

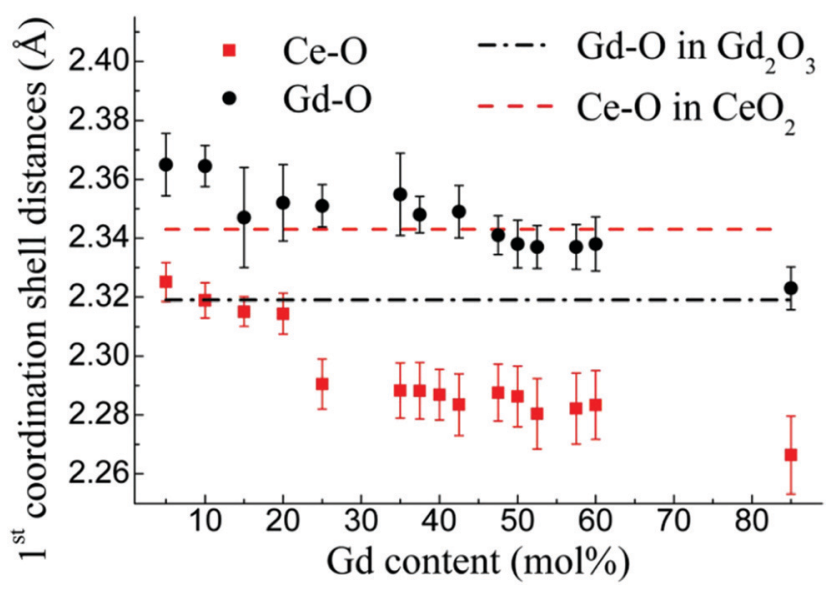

Fig. $16 \mathrm{Ce}-\mathrm{O}$ and $\mathrm{Gd}-\mathrm{O}$ distances in Gd-doped ceria from 5 to 95 mol\% $\mathrm{Gd}$. The top dashed line (red) indicates the $\mathrm{Ce}-\mathrm{O}$ distance for undoped $\mathrm{CeO}_{2}$, while the bottom dashed line (black) indicates the average $\mathrm{Gd}-\mathrm{O}$ distance in $\mathrm{Gd}_{2} \mathrm{O}_{3}$. Both $\mathrm{Ce}-\mathrm{O}$ and $\mathrm{Gd}-\mathrm{O}$ distances are shown to decrease with $\mathrm{Gd}$ content, across the full doping range, with $\mathrm{Ce}-\mathrm{O}$ displaying a sharp change in slope around 20-25 mol\%. Reproduced from ref. 116, with permission from American physical society, copyright 2013.
These regions were defined as "nano-domains" and appear at compositions close to $20 \mathrm{~mol} \%$ for $\mathrm{Gd}$, and close to $30 \mathrm{~mol} \%$ for Sm and $\mathrm{Lu}$. These compositions are at the upper doping levels of the fluorite phase, prior to any detection of doublefluorite by XRD. The evidence presented above suggests that local "double-fluorite - like" regions may exist in the lower mol\% regions normally identified by XRD as "fluorite". As the doping fraction increases towards the XRD detectable phase transition, the double-fluorite-like regions may associate, finally generating nano-domains and later domains large enough to be detected by X-ray diffraction.

The missing link, therefore, for accurately constructing the actual local structure of ceria, may be the consideration of local strain fields, containing Ce-O and Do-O bonds which are much shorter than the average Cat-An distance calculated from the fluorite lattice parameter, similar to distinctly different sets of interatomic distances existing in the double-fluorite structure as described in ref. 143 and 167. Several reports presenting evidence in support of this idea can be cited. Deguchi et al. ${ }^{107}$ suggested, on the basis of Do-Do distances obtained by de-convoluting 2NN EXAFS data for Ce and for Do, that the dopant cation environments resemble the double-fluorite structures in La, Y and Gd-doped ceria. Wang et al. ${ }^{105}$ found that the Y K-edge EXAFS data in $20 \mathrm{~mol} \%$ Y-doped ceria is best modeled by a double-fluorite structure. Kossoy et al. suggested, based on the data in Fig. 16, that the Ce environment in Gd-doped ceria resembles the cation-1 site in the double-fluorite phase.

Additional evidence to the existence of double-fluorite-like disorder prior to detection of phase transition were collected in recent years for a series of doped ceria materials using PDF analysis of neutron and X-ray total scattering data. Similarly to EXAFS, PDF analysis can produce short range interatomic distances. However, $\mathrm{X}$-ray scattering data is insensitive to low atomic mass ions such as oxygen and therefore Cat-Cat distances are predominantly detected. These results summarized in a recent review by Coduri et $a .^{34}$ support the existence of more than one Cat-Cat distance prior to long range detection of the double-fluorite phase in several doped ceria materials. Unlike for EXAFS the identity of the cation is not straightforward to extract, however the analysis supports that the appearance of a longer Cat-Cat distance occurs predominantly around the dopant species. In addition Coduri et al. ${ }^{146}$ using Rietveld refinement of XRD data, detected a small number of cation positions characteristic of the cation-1 site in doublefluorite, at Sm fractions as small as $x=0.125$.

In summary, the appearance of double-fluorite-like local strain fields are related to the decrease in cation ionic radius, as a result of decrease in cation first shell coordination numbers discussed in the previous section. Both effects drive the observed local contraction in Cat-An distances described in Fig. 15. Material properties reported in ceria in recent years, such as electrostriction and anelastic behavior ${ }^{135}$ demonstrated that local strain fields may be active under applied anisotropic stress or electric field. As a result, advanced XANES and particularly EXAFS techniques were employed in order to observe changes in Cat-O distances in situ under an applied electric field. 


\subsection{Modulation of local distortion under bias (electro- mechanical activity)}

An unusually large electromechanical response at room temperature was first reported for Gd-doped ceria in 2012 by Korobko et al. ${ }^{37}$ The strain was found to vary as the square of the electric field; the frequency of the response was second harmonic and therefore the material could be classified as electrostrictive. However, as characterized by Newnham et al. for a large variety of electrostrictive materials, ${ }^{40}$ the electrostrictive strain coefficient should scale with the ratio of the material elastic compliance to the dielectric permittivity. According to this scaling law, materials with large bulk modulus and dielectric constant $\sim 30^{168}$ should not produce a large electrostrictive response. However a response comparable to commercial relaxor materials has been recorded for substrate supported $^{37,39,42}$ and self-supported ${ }^{43}$ doped ceria films as well as for bulk ceramics. ${ }^{160}$ Rather than producing bulk polarization of the material, local, uncorrelated dipolar strain fields were suggested to respond to electric field application. ${ }^{37}$

Techniques allowing for both elemental selectivity and local (first and second shell) structural sensitivity were required in order to test the local strain hypothesis, and to identify the specific lattice sites responsible for strain generation under electric field. XAS techniques, meeting the above requirements, were particularly suitable for the in situ study of doped ceria as a function of applied electric field: conventional XANES, ${ }^{37}$ high energy resolution fluorescence detection (HERFD)-XANES ${ }^{169}$ and "difference X-ray absorption fine structure ( $\triangle$ XANES and $\triangle$ EXAFS), 38 were employed. The information obtained from XANES and HERFD-XANES will be briefly summarized while most of the following will focus on the difference EXAFS data. In addition, we emphasized that all in situ experiments involving application of electric field were to this date performed on magnetron sputtered thin films in a plate capacitor configuration. Small film thickness excludes the need to use high voltage in order to achieve the required electric field.

In the first report describing large electromechanical response in substrate supported films of Gd-doped ceria, ${ }^{37}$ XANES data were collected from the host Ce and dopant Gd species under applied field by scanning the $\mathrm{L}_{\mathrm{III}}$ absorption edges of Ce and Gd cations. No difference in the cation XANES spectra could be detected by measuring E-field ON vs. OFF states. However, applying a combined DC and low-frequency AC bias for several hours (poling), induced a change in the Ce XANES "white line" intensity and shape, when compared to the un-poled state. For the Gd $\mathrm{L}_{\mathrm{III}}$ edge, no such change was detected. This indicated that NN oxygen ligands to Ce changed conformation as a result of the poling process, providing the first evidence for the existence of local strain, and pointing specifically to the Ce sites as its origin. To obtain structural information during ON and OFF states of the field, the sensitivity of the local structure sampling had to be increased. This was achieved by introducing "in situ differential XAS". 38

3.3.1 Difference EXAFS spectra and bond anharmonicity. During electromechanical operation of Gd-doped ceria thin films, the average Cat-O bonds change rather weakly. In order to measure such a small effect, spectra collected during fieldoff are subtracted from spectra collected during field-on, which is called difference XAS (or sometimes “differential XAS”). The resulting difference spectrum is then analyzed and represents the contribution of only the "active species", or the species that were active under the applied stimulus. The XAS spectrum for the "electromechanically active species" in doped ceria can thus be extracted and characterized. These species release and accumulate strain upon electric field modulation. The in situ difference XAS study was performed on sputtered, substrate supported $\mathrm{Ce}_{1-x} \mathrm{Gd}_{x} \mathrm{O}_{2-x / 2}$ films (300-500 nm thick) with $x=0.1,0.2$ and 0.33 , as well as stoichiometric and oxygendeficient ceria: $\mathrm{CeO}_{1.99}$ and $\mathrm{CeO}_{1.96}$, respectively. As mentioned above, thin films are particularly practical for such in situ measurements, since they allow for the application of sufficiently high electric fields $\left(\sim 60 \mathrm{kV} \mathrm{cm}{ }^{-1}\right)$, required for detectable strain generation without the need to apply particularly high voltage. Since the ceramic is placed in a parallel plate capacitor configuration, electrodes must be chosen such that the metal absorption edge does not overlap with the absorption edges of $\mathrm{Ce}$ or $\mathrm{Gd}$, making Ti suitable for these measurements.

XAS spectra for Ce and Gd $\mathrm{L}_{\mathrm{III}}$ edges were collected with bias electric field-on $\left(60 \mathrm{kV} \mathrm{cm}{ }^{-1}\right)$ for $45 \mathrm{~s}$ and field-off for an additional $45 \mathrm{~s}$. These on/off cycles were repeated 10 times. For each half-cycle (on or off), the spectra were normalized and merged (for more details, see ref. 38). For both the XANES and EXAFS regions of the XAS data, the averaged signals in the "off" state were subtracted from the signals in the "on" state, and the resulting difference spectrum was again averaged over time. A difference XANES signal was obtained for the Ce $\mathrm{L}_{\mathrm{III}}$ edge resulting from an intensity increase in the white line upon bias application. No difference in signal was observed for the $\mathrm{Gd}_{\mathrm{III}}$ edge, supporting the result obtained after prolonged modulated field application (poling) in ref. 37, emphasizing that local strain is generated near the Ce cation. The difference XANES signal was qualitatively interpreted as either an increase in the density of unoccupied states of Ce as a result of increased ionic character, stronger configurational order, and/or larger coordination number of neighbors. Most importantly, the largest difference XANES signal for the Ce $\mathrm{L}_{\mathrm{III}}$ edge was obtained for a $10 \mathrm{~mol} \%$ Gd film, which also gave the largest recorded electrostrictive strain coefficient among the measured samples. This emphasized a clear connection between the structural data and the electrostrictive response.

The difference EXAFS signal, $\Delta \chi$, can be defined as:

$$
\Delta \chi=\chi\left(E_{\text {on }}\right)-\chi\left(E_{\text {off }}\right)
$$

where $\chi\left(E_{\text {on or off }}\right)=\chi_{\text {spectator }}+\chi_{\text {active }}\left(E_{\text {on or off }}\right) \cdot \chi_{\text {spectator }}$ is the contribution to the EXAFS signal from photoelectron scattering paths (see eqn (22)) that do not change under applied bias, while $\chi_{\text {active }}$ is the contribution from scattering paths that do change. The difference $k$-weighted EXAFS signal $\Delta \chi$, averaged as described above, is shown in Fig. 17a as a thick black line, and shows a distinct interference pattern, while a single $\chi_{\text {on }}$ EXAFS signal (collected during $45 \mathrm{~s}$ ) is plotted in the background. 
a
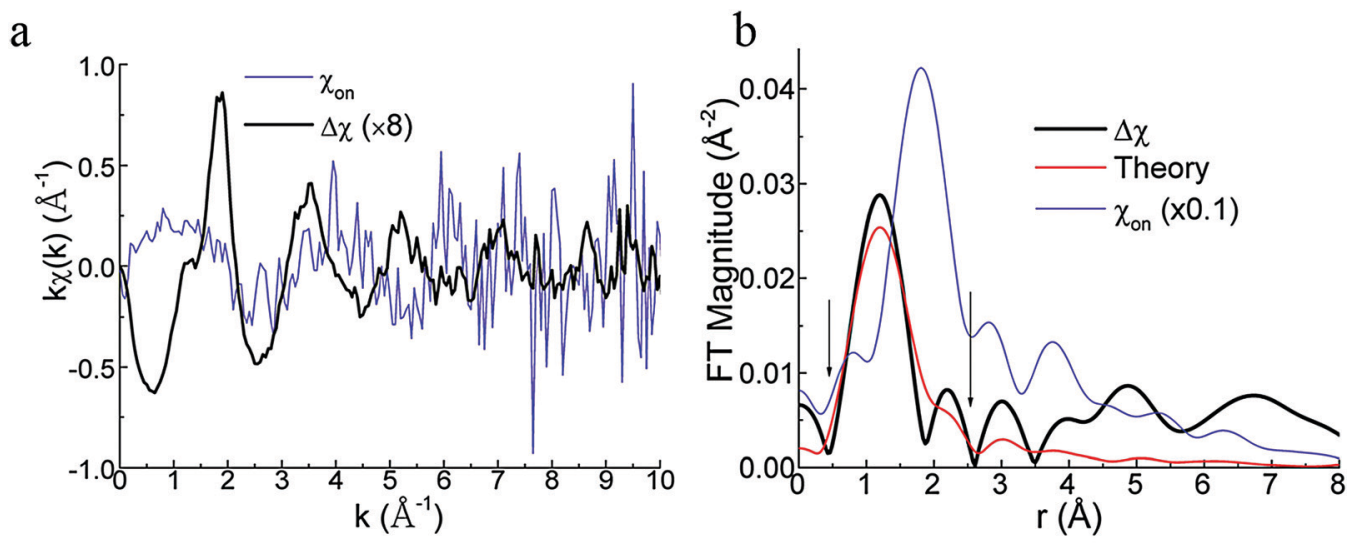

c

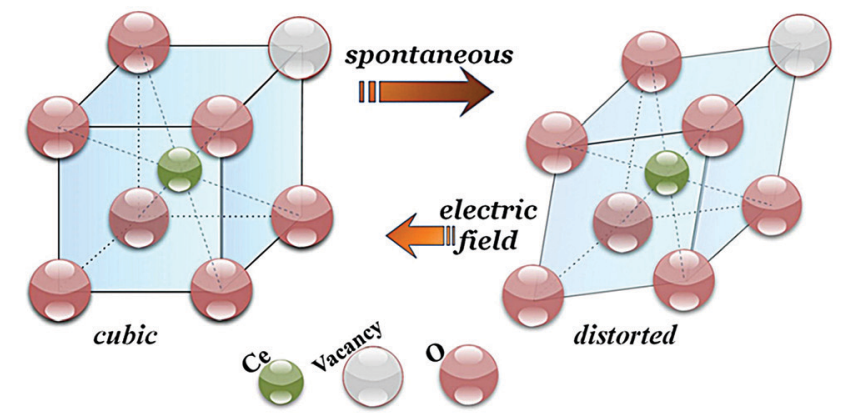

Fig. 17 (a) $k$-Weighted EXAFS $\chi_{\text {on }}$ signal recorded during $45 \mathrm{~s}$ of field application (one half cycle), and the difference EXAFS signal $\Delta \chi$, acquired according to eqn (27), representing the signal due to the "active species". (b) RDF plot for the general Ce-O bond population represented by $\chi_{\text {on, }}$ and the "active" $\mathrm{Ce}-\mathrm{O}$ bond population represented by $\Delta \chi$. (c) An illustration of distorted cation sites (right) in the crystal as a result of decreased coordination number around the cation site from 8 to 7 oxygen atoms. When an electric field is applied, such units change towards the undistorted form, generating macroscopic strain. Adapted from ref. 38 with permission from American Institute of Physics, copyright 2015.

The first strong peak of the radial distribution function (RDF) represents information concerning Ce-O distances (Fig. 17b). By comparing the Ce-O RDF peak for $\Delta \chi$ - representing the active bonds, to the $\chi_{\text {on }}$ RDF (an average of all "on" half-cycles), we can qualitatively see that the active $\mathrm{Ce}-\mathrm{O}$ bonds are shorter than the spectators. In order to fit $\Delta \chi$ ('active' bond signal) and extract the EXAFS parameters, it was necessary to consider the active bond "anharmonicity". This is accomplished by including higher order terms in the cumulant expansion that give rise to the structural parameters, such as the coordination number $N$, the effective nearest-neighbor distance $r$ and the Debye Waller factor term $\mathrm{e}^{-2 k^{2} \sigma^{2}}$ of eqn (22). A skew in the radial distribution caused by anharmonicity of the interatomic potential ${ }^{132,133}$ requires corrections to the Gaussian form of the Debye-Waller factor. ${ }^{122}$ The third cumulant, $C_{3}$, characterizes the skew of the radial distribution function and is introduced as a correction to the phase term $\delta(k)$ in the EXAFS equation of a single scattering path:

$$
\chi(k)=S_{0}^{2} N \frac{f(k)}{k r^{2}} \mathrm{e}^{-\frac{2 r}{\lambda(k)}} \mathrm{e}^{-2 k^{2} \sigma^{2}} \sin \left(2 k r-\frac{4}{3} C_{3} k^{3}+\delta(k)\right) .
$$

Parameters $N, r$ and $C_{3}$ were extracted for active Ce-O bonds and spectators. The averaged active $\mathrm{Ce}-\mathrm{O}$ bonds in the difference EXAFS signal were found to be $2.22 \pm 0.09 \AA$ in length, which is $4.6 \%$ shorter than the average $\mathrm{Ce}-\mathrm{O}$ bonds in the material $(2.33 \pm 0.02 \AA)$. The active bond anharmonicity factor
$C_{3}$ was found to be unusually large $\left(0.210 \pm 0.008 \AA^{3}\right)$, compared to the negligibly small value $\left(0.000 \pm 0.002 \AA^{3}\right)$ for the spectator bonds. Comparison of coordination numbers suggested short bonds comprise $\approx 3.4 \%$ of the total $\mathrm{Ce}-\mathrm{O}$ bonds. This was consistent with the value of macroscopic strain $\approx-6 \times 10^{-4}$ measured during electrostrictive actuation. Finally, a negative sign in the difference coordination number $N$ for $\Delta \chi$, indicated that short bonds are not present in the "on" state. The general conclusion from this study is that local distortions (strain fields on the interatomic level) are present in the material prior to field application, comprising, in part, short, anharmonic $\mathrm{Ce}-\mathrm{O}$ bonds. Upon field application, the distortions are minimized, thus generating measurable macroscopic strain, as illustrated in Fig. 17c. Although the model of the local distortion and the active $\mathrm{Ce}-\mathrm{O}$ bonds was speculated to resemble the double-fluorite cation- 1 site, ${ }^{116}$ HERFD results, described briefly below, indicated that local distortions prior to field application include a combination of configurations, not necessarily a single, well defined, double-fluorite - like structure.

3.3.2 High-resolution XANES-HERFD. XANES was revisited in an attempt to resolve the distorted ligand structure. To do so, Li et al. ${ }^{169}$ used the "high energy resolution fluorescence detection (HERFD) XANES" technique, applying a prolonged (several hours) modulated field, similar to that described at the beginning of this Section 3.3 in ref. 37. This allowed acquisition of the $\mathrm{Ce}_{\mathrm{III}}$ white line profile in its finest detail, shown 
a

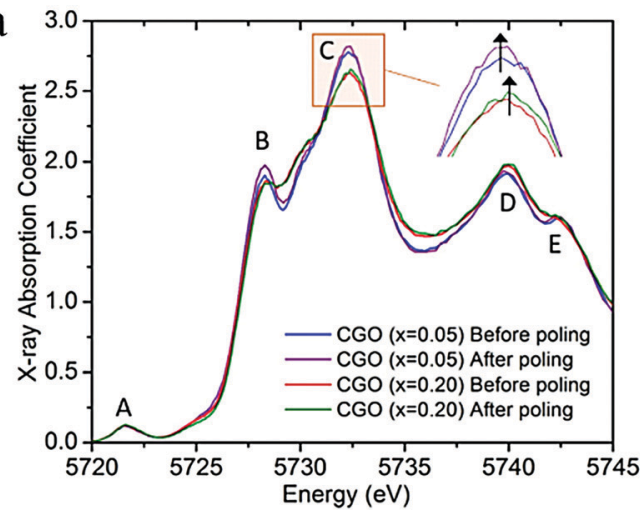

b

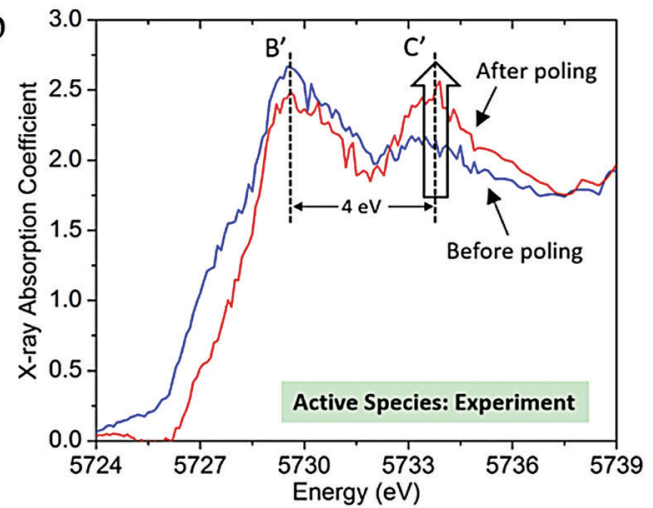

M1

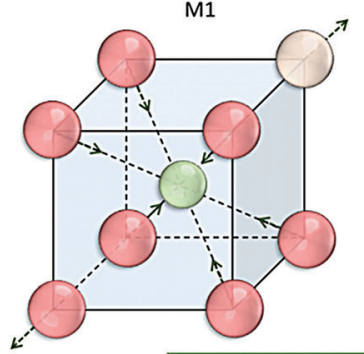

M2

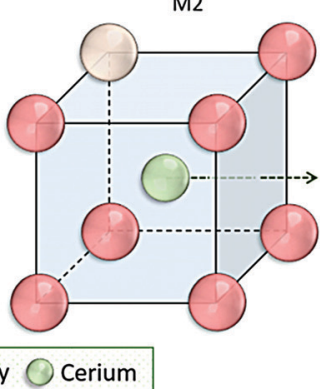

Fig. 18 (a) HERFD white lines for the Ce absorption edge of 5 and 20 mol\% ceria films, before and after "poling" with a combination of AC and DC fields, $V_{\text {applied }}=V_{\mathrm{DC}}+V_{\mathrm{AC}} \cdot \cos (2 \pi f t)\left[f=10 \mathrm{mHz}, V_{\mathrm{AC}}=0.75 \mathrm{~V}, V_{\mathrm{DC}}=0.4 \mathrm{~V}\right]$. (b) Isolating the contribution of the active species to the intensity change in the "white line" peaks. (c) A model which combines two different local distortions is required in order to reproduce the intensity change caused by the active species to the Ce white line. Adapted from ref. 169 with permission from American Institute of Physics, copyright 2016.

in Fig. 18a. As described in Fig. 8 of Section 3.1, the Ce $\mathrm{L}_{\mathrm{III}}$ white line displays multiple peaks due to a combination of mixed final states and crystal field splitting. At high resolution, all 4 peaks are evident as shown in Fig. 18a, with peaks B and C corresponding to peaks 3 and 2 in Fig. $8 \mathrm{~b}$, and the high resolution capturing the splitting of peak 1 of Fig. $8 \mathrm{~b}$ as peaks $\mathrm{D}$ and $\mathrm{E}$ in Fig. 18a. ${ }^{120,170}$ The contribution of the active species to the intensity changes under poling was then isolated Fig. $18 \mathrm{~b}$ by assuming that all acquired spectra for both Gd concentrations ( $5 \mathrm{~mol} \%$ and $20 \mathrm{~mol} \%$ ) are linear combinations of spectra for active and spectator species (detailed in ref. 169). Based on the mechanism described in Fig. 17c, following application of an electric field, the active species environment returned to more symmetric form which is represented by the spectrum "After poling" in Fig. 18b. A model combining atomic shifts along $\langle 111\rangle$ in the anion sub-lattice (Fig. 18c, LHS) and shifts in the Ce cation position along $\langle 100\rangle$ (Fig. 18c, RHS) was necessary in order to reproduce the spectrum for "Before poling" which produces a relative change in intensity of peaks $\mathrm{B}^{\prime}$ and $\mathrm{C}^{\prime}$ as observed experimentally in Fig. 18b.

\subsection{Correlating long range and short range data in a single model approach}

Recently, a Reverse Monte Carlo (RMC) based approach was implemented to the analysis of undoped and doped ceria EXAFS data. ${ }^{58,171}$ The basic principle involves the extraction of a theoretical EXAFS spectrum from a simulated 3D model structure. The structure then continues to evolve through random atomic displacements, until theoretical and experimental spectra reach maximal agreement. The implementation of the RMC approach to EXAFS data improves the ability of data extraction beyond the first and second coordination shells from the EXAFS spectrum and tackles issues like "multiple scattering.". ${ }^{172}$ Besides the advantages for EXAFS analysis, the potential of an RMC based approach for ceria is the ability to constrain the model with several sets of experimental data, as implemented for amorphous materials in the past, ${ }^{173}$ representing short, intermediate and long range data, from neutron or X-ray total scattering together with EXAFS, consolidating the apparent inconsistencies between them. For ceria materials RMC models where constructed separately for neutron diffraction data of undoped ${ }^{83}$ and $\mathrm{Nb}$ or Ca doped ${ }^{174}$ ceria. In a step towards multiscale modeling, Clark et al. ${ }^{171}$ employed RMC modeling to EXAFS as well as neutron total scattering to model undoped ceria powder material. More recently XRD lattice parameters and EXAFS data were consolidated in a single model structure, ${ }^{58}$ for a series of Y- and Sm-doped ceria solid solutions. Here sputter deposited thin films were investigated. This allowed the assumption of randomly distributed solid solutions, since deposition on cold substrates reduces the possibility for preferential defect clustering at low doping fractions. The model showed that in order to produce realistic $\mathrm{Ce}-\mathrm{V}_{\mathrm{O}}$ distances and also for the average XRD and local EXAFS experimental data to co-exist in the same crystal structure, first 
shell (Cat-O) and second shell (Cat-Cat) distances must display bimodal distributions. Specifically, more than one bond population is suggested to exist for $\mathrm{Ce}-\mathrm{O}$, Do-O and Ce-Cat distances, where for dopant coordination shells, the split in population was observed even for doping fractions as low as $5 \mathrm{~mol} \%$. This finding supports the existence of local doublefluorite like arrangements in the vicinity of a dopant as well as Ce host cations, see discussion in Section 3.2.6. From a functional perspective it supports the existence of local strain fields, able to deform under electric field, as discussed in Section 3.3. In addition, when discussing ion conductivity it implies that a basic diffusion step, a jump of an oxygen-vacancy does not have an equal probability for all six neighboring oxygen sites but may have some "preferential" directions caused by local asymmetry. The above experimental works sample the potential of the RMC approach for constructing multiple scale models for doped ceria materials.

\subsection{Conclusions from EXAFS: local structure of ceria solid solutions}

Why use EXAFS? Ceria solid solutions, their crystallographic phases and structure, are primarily analyzed in the literature via $\mathrm{XRD}$, probing the average long-range periodic ordering of cations. The commonly accepted view is that interatomic distances in ceria solid solutions can be described by a fluorite space group for $\leq 20 \mathrm{~mol} \%$ trivalent rare earth dopant. In this section, we have focused on the results of X-ray absorption fine structure (XAFS) spectroscopy techniques: EXAFS and XANES, for ceria solid solutions doped with trivalent cations. These techniques possess several advantages over conventional X-ray diffraction in being capable of probing the local bonding environment of ceria and its solid solutions, giving useful information on coordination environments of neighbors and next nearest neighbors in the atomic structure. Additionally, in contrast to X-ray diffraction, XAFS is an element-specific probe of local structure, which can characterize lattice distortions and defects.

Comparison of XRD and EXAFS data suggests local deviation from fluorite symmetry. Ion packing models relying on fluorite symmetry are not able to reproduce the discrepancy, revealed by EXAFS, where Ce-Ce distances are larger than Do-Ce distances for some dopants that are larger than $\mathrm{Ce}^{4+}$. In addition, EXAFS- and XRD-derived interatomic distances disagree and result in the calculation of unphysical Cat $-\mathrm{V}_{\mathrm{O}}$ distances under the assumption of a uniform fluorite phase. This finding is supported by the combination of EXAFS and XRD data in a simulated structure (using reverse Monte-Carlo-EXAFS modelling ${ }^{58}$, which reveals bi-modal distance distributions for Ce-O, Do-O and Ce-Do. The assumption of local fluorite symmetry must therefore be incorrect for ceria solid solutions at low-to-moderate doping levels. This observation is reminiscent of contradictions found between XRD and EXAFS data collected for several other randomly mixed oxide and other mixed ionic compounds, ${ }^{148,150,175,176}$ which were interpreted as interatomic bond-buckling effects. More specifically, EXAFS analysis of the full doping range, from fluorite to doublefluorite, indicated angular deformations even with dopant concentration $<30 \mathrm{~mol} \%$, where only the pure fluorite phase is detected by XRD. These deformations may locally resemble the double-fluorite phase found at higher doping fractions. This, and the evidence provided by additional techniques such as Raman spectroscopy and TEM imaging, support a gradual, rather than abrupt, fluorite to double-fluorite transition at approx. $30 \mathrm{~mol} \%$, with increase in dopant content.

Effect of dopant choice and concentration on local structure and lattice distortions. For trivalent dopants with ionic radius $>105 \mathrm{pm}$ (e.g. $\mathrm{Gd}^{3+}, \mathrm{Sm}^{3+}, \mathrm{Nd}^{3+}$ ), the lattice parameter increases with doping, although the average Cat-O distance contracts (for both Ce and Do first shell), as determined from EXAFS and PDF analyses. This contradiction is explained in part by a reduction in cation nearest neighbor (1NN) coordination number through appearance of oxygen vacancies with increasing dopant content and, in part, as mentioned above, by local deviations from fluorite $F m \overline{3} m$ symmetry. However varying degrees of defect interaction and defect ordering play an important part as well: dopants that are larger than $\mathrm{Nd}^{3+}$ and smaller than $\mathrm{Gd}^{3+}$ display different relationships between the local and average structures. $\mathrm{La}^{3+}$ for example, is larger than $\mathrm{Nd}^{3+}$ and La-doped ceria solid solutions display expansion in the lattice parameter with dopant content. However, contraction in EXAFS-derived Cat-O is observed only for Ce-O distances while La-O follows the symmetry-predicted trend. This also provides evidence that local deformation as a result of oxygen-vacancy formation is present mainly near $\mathrm{Ce}^{4+}$. For dopants with ionic radius smaller than $\mathrm{Gd}^{3+}$, e.g. , $\mathrm{Y}^{3+}, \mathrm{Er}^{3+}$ and $\mathrm{Yb}^{3+}$ the average and local environments qualitatively agree by producing contraction of lattice parameters as well as Cat-O distances.

Role of local strain fields in ceria mechanical and electromechanical properties. Some macroscopic properties of doped ceria, such as unusually large electrostrictive strain coefficients and room temperature anelastic behavior, ${ }^{135}$ also support local deviation from fluorite symmetry, which produces anisotropic stress via reorganization of local dipolar strain fields.

These observations provided a new approach by suggesting that, through the application of an external electric field in combination with XAS, the state of local strain fields in the vicinity of point-defects could be detected. Local cation environments were probed by XANES, ${ }^{37}$ EXAFS, ${ }^{38}$ and HERFD $^{169}$ before, during and after the application of the electric field under "poling" and low-frequency modulation regimes. These measurements have shown that: (i) a small number of $\mathrm{Ce}^{4+}$ ions have non-fluorite, 1NN oxygen coordination shells containing "active" bonds which can be modified by electric field or stress application; (ii) active Ce-O bonds are strained by $\sim-4.6 \%$ (at $2.22 \AA)$ with respect to their length in fluorite ceria ( $2.33 \AA$ ); the response to an applied electric field is expansion i.e., return to a cation-anion bond length closer to that expected from fluorite symmetry. The recent experiments described above highlight the role of modulated electric field application to selectively activate minority electroactive bonds, enhancing the sensitivity of XAFS experiments to their environment. ${ }^{38,169,177}$

What next? The capabilities of XAS techniques have yet to be fully explored for ceria solid solutions. With the exception of 
Gd, most rare earth trivalent dopants, such as Sm, Nd, La, have not been systematically investigated by XAS across their full doping range. This presents researchers with the opportunity of uncovering a fascinating pattern of point-defect local strain fields and interactions which depend on both the dopant concentration and size. Understanding the above effects is essential for many practical applications of doped ceria; and, to this end, we encourage the vigorous development of models which are constrained by both XRD and EXAFS data but which do not assume perfect local fluorite arrangements: one such method is RMC-EXAFS, described above. Only in this way, and with the added assistance of Raman $\mu$-spectroscopy, can we hope to finally arrive at an atomic level description of the not-so-perfect, but real, lattice structures of ceria solid solutions.

\section{Raman spectroscopy for probing point-defects and lattice distortions in undoped and doped ceria}

Raman spectroscopy is a versatile and readily accessible experimental technique to investigate bonding and short-range order in metal-oxide materials by detecting lattice vibrations. In the following sections, we shall describe how pressure, temperature, oxygen non-stoichiometry, and extrinsic doping influence the Raman spectrum of ceria and its solid solutions, and how this information can be used to understand the defectchemistry and local defect structures. Raman spectroscopy is also an excellent tool for characterizing the electro-chemomechanical properties of ceria, facilitated by in situ application of anisotropic stress or electric fields. In the following, we first introduce the fundamentals of Raman spectroscopy and then provide an overview of the interpretation of Raman spectra in ceria, with focus on point defects induced by aliovalent doping and oxygen non-stoichiometry.

\subsection{Basics of Raman spectroscopy in solid state compounds}

Raman spectroscopy is a form of vibrational spectroscopy that measures the frequency and Raman intensity of lattice oscillation modes in solids. ${ }^{178}$ It overcomes the major drawback of XRD for oxides, which is the weak X-ray scattering amplitude of oxygen and the relative insensitivity to uncorrelated point-defects.
Raman scattering is an inelastic scattering of a photon by a lattice oscillation (phonon). By this process, the frequency of the scattered photon shifts by an amount equal to the phonon frequency, which enables measurement of the frequencies of symmetry permitted interatomic bond vibrations. For the characterization of solids, usually confocal optical microscopes are used, and objective lenses have very high numeric aperture for efficient collection of the rather weak Raman scattering signal. The spatial resolution limit of this detection method is around $500 \mathrm{~nm}$ and $1 \mu \mathrm{m}$ in depth. By depositing plasmonic structures on a substrate, the information depth can be reduced to few nanometers, which is called surface enhanced Raman spectroscopy. ${ }^{179}$ Another approach for increasing spatial resolution is tip-enhanced Raman spectroscopy (TERS), which uses an Atomic Force Microscope (AFM) tip few nanometers above the sample which enhances the field strength below the tip. ${ }^{180,181}$ Not only the spatial but also the temporal resolution of Raman spectroscopy can be enhanced by so-called femtosecond stimulated Raman spectroscopy, ${ }^{182,183}$ which enables the characterization of excited states. These advanced detection modes show the versatility of Raman spectroscopy. In standard configurations, the sample is illuminated with monochromatic light, typically a laser in the near-IR, visible or near-UV range. As shown in Fig. 19, a laser photon excites an electron into a virtual energy level with an extremely short lifetime. Upon relaxation, the emitted photon may have the same energy (elastic (Rayleigh) scattering), or the energy may be shifted by the energy of a phonon mode (Stokes, anti-Stokes scattering).

The macroscopic scattering process is described as follows. The incident laser light may be characterized by the electric field vector $\vec{E}$ of (angular) frequency $\omega_{0}$. Since the wavelength of the light is much longer than interatomic distances, its $k$-vector can be approximated as zero, described by the equation

$$
\vec{E}=\overrightarrow{E_{0}} \cos \left(\omega_{0} t\right)
$$

where $t$ is time. If we consider a Raman-active bond of interest in our sample with a given vibrational frequency $\omega_{\mathrm{vib}}$, the bond length $x$ oscillates around its equilibrium position $x_{0}$,

$$
x=x_{0}+A \cos \left(\omega_{\mathrm{vib}} t\right) .
$$

where $A$ is the vibration amplitude. The electric field of the incoming light polarizes this oscillating bond, inducing a dipole moment p. In the case of conventional Raman spectroscopy

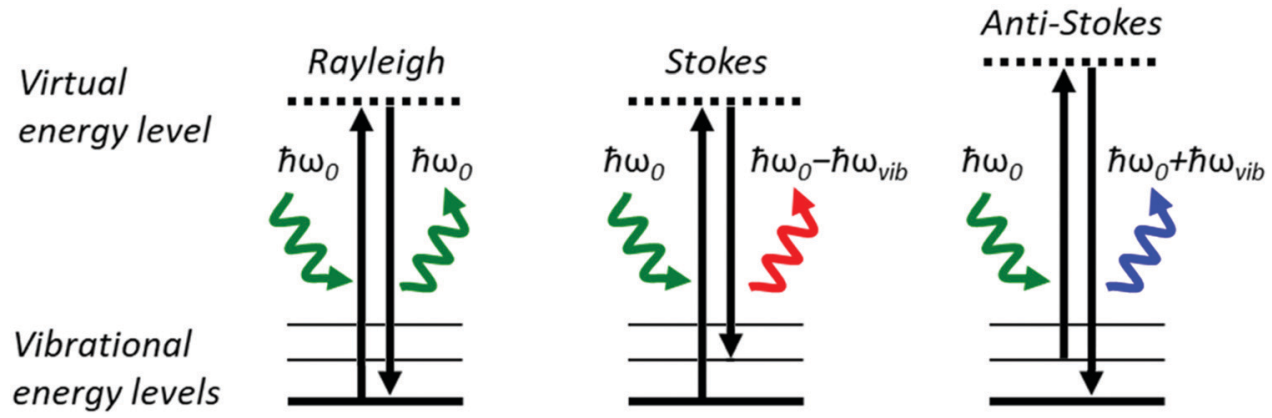

Fig. 19 Schematic representation of the energy levels involved in Rayleigh, Stokes, and anti-Stokes Raman scattering. 
with moderate light intensities, the dipole moment is proportional to the electric field times polarizability $\alpha$ :

$$
\mathbf{p}=\alpha \cdot \vec{E}
$$

When atoms in the lattice vibrate around their equilibrium position, the polarizability changes as well, and may be linearized for small amplitude as

$$
\boldsymbol{p}=\left(\alpha_{0}+\left.\frac{\partial \alpha}{\partial x}\right|_{x_{0}}\left(x-x_{0}\right)\right) \vec{E}
$$

In this sense, Raman scattering occurs through the modulation of the polarizability due to lattice vibration. Inserting eqn (30) and (31) into eqn (32) yields

$$
\boldsymbol{p}=\alpha \overrightarrow{E_{0}} \cos \left(\omega_{0} t\right)+\left.A \frac{\partial \alpha}{\partial x}\right|_{x_{0}} \overrightarrow{E_{0}} \cos \left(\omega_{0} t\right) x_{0} \cos \left(\omega_{\mathrm{vib}} t\right)
$$

We may rewrite the equation using the trigonometric relation $\cos (\alpha) \cos (\beta)=\frac{1}{2}(\cos (\alpha-\beta)+\cos (\alpha+\beta))$, which yields

$$
\begin{aligned}
& \boldsymbol{p}= \\
& \overrightarrow{E_{0}}\left(\alpha_{0} \cos \left(\omega_{0} t\right)+\left.\frac{1}{2} A \frac{\partial \alpha}{\partial x}\right|_{x_{0}}\left(\cos \left(\omega_{0} t-\omega_{\mathrm{vib}} t\right)+\cos \left(\omega_{0} t+\omega_{\mathrm{vib}} t\right)\right)\right)
\end{aligned}
$$

Eqn (34) shows that in addition to the frequency of the incoming light, Raman scattering gives rise to two new frequencies, $\omega_{0}-\omega_{\text {vib }}$ (Stokes scattering) and $\omega_{0}+\omega_{\text {vib }}$ (antiStokes scattering), whenever the partial derivative of the polarizability $\left.\frac{\partial \alpha}{\partial x}\right|_{x_{0}}$ in eqn (32) is non-zero. This defines the selection rule for Raman activity since a vibrational mode is Raman active only if it induces a first order change in the polarizability. In other words, only when a lattice oscillation mode causes variations of the polarizability at the same frequency, this oscillation is first order Raman active.

Some general features of Raman spectroscopy are relevant for any Raman experiment. (1) Vibrational energy levels are occupied according to Boltzmann statistics, and therefore Stokes scattering from the ground state to an excited state is more intense than anti-Stokes scattering from the less populated excited state. By calculating the ratio of the Stokes and anti-Stokes bands, the sample temperature may be determined. ${ }^{184,185}$ (2) Only when the lattice oscillation has the same phase for neighboring unit cells, this oscillation is Raman active. In the phonon dispersion curves, this condition is only satisfied at the Brillouin zone center, where the reduced $k$ vector equals 0 , also denoted as $\Gamma$. This second condition is the reason why Raman peaks in crystalline solids can be quite narrow. (3) Although the Raman shift of a vibrational mode is independent of the exciting laser wavelength, Raman scattering amplitude may vary strongly with wavelength. Resonant intensity enhancement occurs when the exciting photon energy is close to the energy of an electronic transition, which strongly increases the lifetime of the photo-excited state, also known as resonant Raman scattering: ${ }^{186}$ Since excitation of real electronic states often also increases fluorescence, resonant enhancement does not increase the signal to background ratio in all cases.

Materials doped e.g. with either Er or Eu often exhibit strong fluorescence signals, which are typically much stronger than the detectable Raman scattering signal. ${ }^{187-189}$ An example of fluorescence in Er doped Ceria is shown in ESI, $\dagger$ Fig. S2.3. In contrast to Raman scattering, the wavelength of fluorescence signals is independent of the exciting laser wavelength, and in most cases also strongly fluorescent materials can be investigated by selecting the appropriate laser wavelength, as exemplified on measurements by the Rupp group in the ESI, $\uparrow$ Section S2.3. Actually fluorescence signals can yield details on the local environment of fluorescent ions and can be effectively used to gain additional local near order information on structures, e.g. see for example on the use of $\mathrm{Eu}^{3+}$ ref. 190 for further insights.

As noted above, vibrational modes are only Raman active when a lattice oscillation causes a first order change in the electric polarizability. Raman active vibrations can be predicted by group theory, taking into account the crystallographic symmetries. ${ }^{191}$ Typically, crystal structures with high symmetry have few Raman active oscillation modes. However, lattice distortions introduced by doping or oxygen-vacancy defects can locally lift the inversion symmetry and therefore introduce Raman activity also for normally inactive oscillation modes. Moreover, the Raman vibrational frequency depends on lattice geometry and bonding, specifically the bond stiffness, atomic mass and coordination number. In the following sections we will discuss these effects for ceria.

As detailed in Section 1, undoped ceria crystallizes in the cubic fluorite $F m \overline{3} m$ structure (point group $m \overline{3} m$ ), shown in Fig. 1. Due to the high symmetry of the crystal structure, only one triply degenerate oxygen breathing mode of $\mathrm{F}_{2 \mathrm{~g}}$ symmetry at $465 \mathrm{~cm}^{-1}$ is Raman active, ${ }^{192}$ as shown in Fig. 20. Analogous to infrared spectroscopy, the Raman shift is typically measured in inverse wavelength, with the relation $\frac{1}{\lambda}=\bar{\nu}=\frac{f_{\text {vib }}}{c}$, where $\lambda$ is the wavelength, $\bar{\nu}$ the wavenumber, $f_{\text {vib }}$ the (temporal) vibrational frequency, and $c$ the speed of light.

In crystalline solids, lattice vibration frequencies are typically displayed on phonon dispersion curves. Phonon dispersion curves can be measured by inelastic neutron scattering, ${ }^{193}$ inelastic X-ray scattering, ${ }^{194}$ or high-resolution electron energy loss spectroscopy EELS. ${ }^{195}$ They can also be calculated by density functional theory (DFT). ${ }^{196}$ The phonon dispersion curves for $\mathrm{CeO}_{2}$ are shown in Fig. 21, where the experimentally measured data are superimposed on the DFT-calculated curves.

For vibrational spectroscopy, only the Brillouin zone center frequencies, denoted by the point $\Gamma$ in Fig. 21 are of interest. Other points in the wave vector axis $(X, K, L, W)$ designate edges and corners of the Brillouin zone, but are not of interest here, because in ceria these oscillations produce very weak second order Raman active modes. ${ }^{198}$ The $F m \overline{3} m$ space group (cubic fluorite) of ceria gives rise to six partially degenerate optical phonon modes yielding three frequencies at the $\Gamma$ point. Out of all 


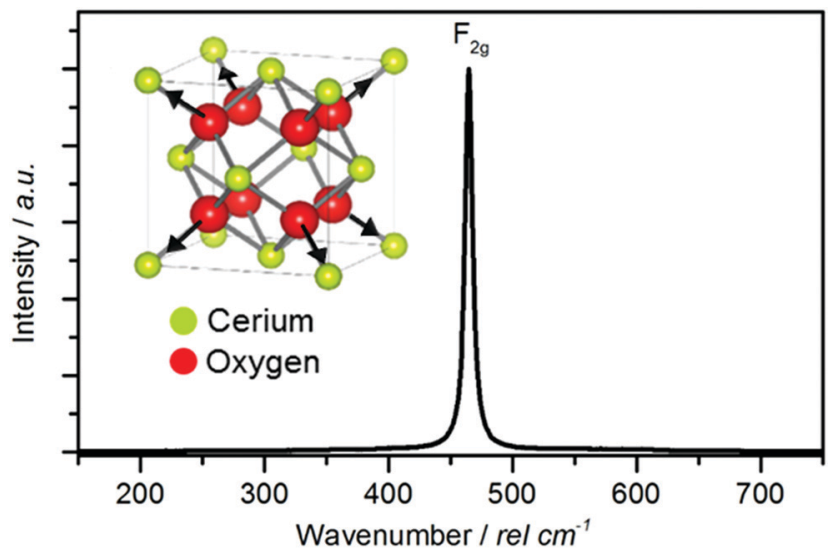

Fig. 20 Raman spectrum of oxidized $\mathrm{CeO}_{2}$ showing only one single Raman active mode with $\mathrm{F}_{2 \mathrm{~g}}$ symmetry. According to its group theory, undoped ceria shows only one triply degenerate Raman active mode, in which oxygen atoms vibrate around the cation, which is stationary. The crystal structure and the $F_{2 g}$ bond vibrations are shown in the inset.

optical phonon modes, as noted above, only the triply degenerate $F_{2 g}$ mode is Raman active, see Fig. $20 .{ }^{198}$ The $F_{2 g}$ mode is the symmetric breathing vibration of the tetrahedrallycoordinated oxygen ions about the unit cell center. Since cations do not oscillate, the frequency is independent of the cationic mass. ${ }^{61}$ On the other hand, any variation in bond length or the formation of oxygen-vacancy defects will have a direct impact on the oscillation frequency and are therefore detectable in the Raman spectra. ${ }^{60}$ The two other optical phonon modes visible in Fig. 21 with Brillouin zone center frequencies corresponding to $270 \mathrm{~cm}^{-1}$ and $595 \mathrm{~cm}^{-1}$ have $F_{1 \mathrm{u}}$ symmetry and are therefore IR but not Raman-active.

\subsection{Interpretation of temperature or isostatic pressure} induced changes in the $F_{2 g}$ mode in terms of defect structure and chemistry

Raman spectroscopy is an excellent technique for probing the chemo-mechanics of a solid. Both the isothermal and isobaric

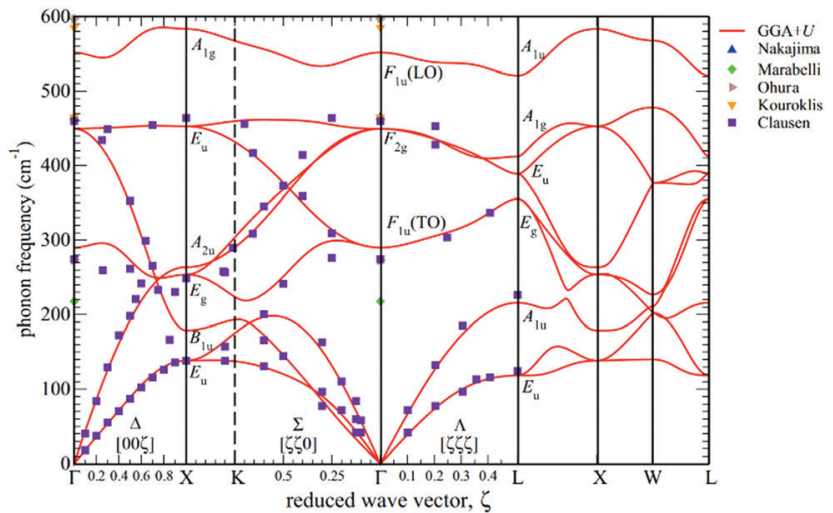

Fig. 21 Phonon dispersion curves of $\mathrm{CeO}_{2}$. The data lines were calculated by DFT and the data points measured by inelastic neutron scattering (purple squares), Raman scattering (blue and orange triangles), infrared spectroscopy (brown triangle), and reflectivity (green diamond). Reproduced from ref. 197, with permission from American Physical Society, copyright 2013.
Grüneisen parameters, accessible via Raman spectra, describe among other parameters the relationship between unit cell volume and lattice vibrational frequency. The effects of isostatic pressure on Raman spectra have been reviewed by Lucazeau. ${ }^{199}$ In the Grüneisen model, the frequency of a Raman mode shifts when the lattice expands or contracts. Due to the anharmonicity of the lattice potentials, shorter bonds are stiffer and therefore cause higher oscillation frequencies. For a cubic crystal lattice, the unit cell parameter $a_{0}$ and vibrational frequency $\omega_{0}$ are related by:

$$
\frac{\Delta \omega}{\omega_{0}}=-\gamma \frac{\Delta V}{V_{0}} \approx-3 \gamma \frac{\Delta a}{a_{0}},
$$

where $\omega_{0}, V_{0}$ and $a_{0}$ are vibrational frequency, unit cell volume and lattice parameter under STP, and $\Delta \omega, \Delta V$ and $\Delta a$ are changes with pressure or temperature. Within the quasiharmonic approximation, the dimensionless Grüneisen parameter $\gamma$ should be the same for isothermal and isobaric lattice parameter changes. For changes in hydrostatic pressure we can define the isothermal Grüneisen parameter as

$$
\gamma_{\mathrm{T}}=\left(\frac{B}{\omega_{0}}\right) \frac{\mathrm{d} \omega}{\mathrm{d} P}
$$

where $B$ is the bulk modulus and $\mathrm{d} \omega / \mathrm{d} P$ the shift in the Raman frequency. For temperature dependent measurements, we obtain for the isobaric Grüneisen parameter

$$
\gamma_{\mathrm{P}}=\left(\frac{-1}{\alpha_{\mathrm{T}} \omega_{0}}\right) \frac{\mathrm{d} \omega}{\mathrm{d} T},
$$

where $\alpha_{\mathrm{T}}$ is the volumetric thermal expansion coefficient and $\mathrm{d} \omega / \mathrm{d} T$ the shift in the Raman frequency with temperature. In Fig. 22, examples of pressure dependent and temperature

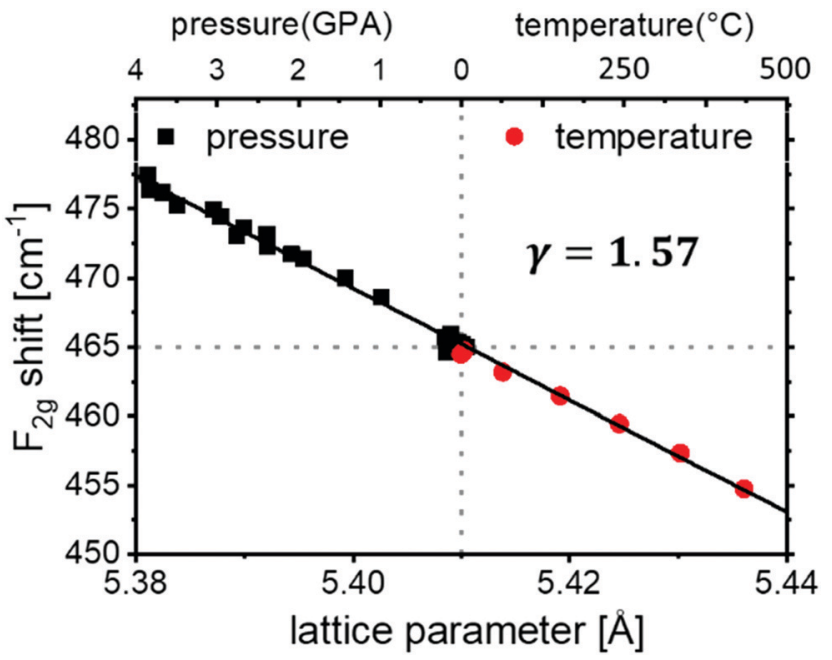

Fig. 22 Position of the $\mathrm{F}_{2 \mathrm{~g}}$ mode as a function of the lattice parameter in $\mathrm{CeO}_{2}$. Black square data points were measured at high hydrostatic pressure for $\mathrm{CeO}_{2}$ powder, taken from ref. 201, and lattice parameter changes were calculated using a bulk modulus of $230 \mathrm{GPa}{ }^{202}$ Red circle data points were measured on heated $\mathrm{CeO}_{2}$ pelletsat ambient pressure and the thermal expansion coefficient was taken from ref. 203, see ESI, $\dagger$ Section S2 for details. 
dependent Raman measurements are demonstrated. Several groups have investigated the $\mathrm{F}_{2 \mathrm{~g}}$ frequency shift of ceria under hydrostatic pressure. For high pressure Raman spectroscopy, the sample is typically enclosed in a high pressure diamond anvil cell. ${ }^{200}$ For example, Li et al. ${ }^{201}$ measured the pressure dependent $F_{2 g}$ peak position of large grained ceria, the values are shown in Fig. 22 (black squares). A pressure dependent lattice parameter was calculated by taking $230 \mathrm{GPa}$ as the bulk modulus of $\mathrm{CeO}_{2}$ under ambient conditions. ${ }^{202}$ With increasing pressure, an increase in vibrational frequency (due to increasing stiffness of compressed bonds) of $3.2 \pm 0.1 \mathrm{~cm}^{-1}$ per GPa was observed. ${ }^{200}$ Temperature dependent data were acquired by the Rupp group on sintered ceria pellets with grain size of $\sim 2 \mu \mathrm{m}$ on a temperature calibrated button heater, see ESI, $\dagger$ Section S3.1.2 for details. The lattice parameter was calculated according to the thermal expansion coefficient taken from ref. 203. As noted in Section 2, thermal reduction of ceria in air is negligible up to at least $1000{ }^{\circ} \mathrm{C}$, and thus does not contribute to the lattice expansion that otherwise results upon reduction. ${ }^{56}$ The fact that the Grüneisen parameter of 1.57 fits both the high pressure and high temperature data indicates that undoped ceria is quasi-harmonic for the temperature range.

It is important to note that the case presented here is unusual in its ideality. As discussed by Lucazeau, ${ }^{199}$ the temperature dependent Raman shift can be affected by more than just thermal expansion and may therefore deviate from linearity, as well as from the value for the isothermal Grüneisen parameter. In addition, factors such as multiple scattering processes must be considered in some cases where deviations from the quasi-harmonic approximation are found. These effects are addressed for example in ref. 204 and 205, where nanocrystalline or oxygen-deficient ceria is considered. An additional important point is significant variation of the reported isothermal Grüneisen parameter in the literature, in part due to use of different values for the bulk modulus: some authors used the bulk modulus of $\mathrm{ThO}_{2} 204 \mathrm{GPa}$ (instead of $230 \mathrm{GPa}$ which was measured for $\mathrm{CeO}_{2}{ }^{202}$ ) and claim a value of $\gamma=1.44^{200}$ for $\mathrm{CeO}_{2}$ single crystals. For nano-crystalline $\mathrm{CeO}_{2}$ powders, values range from $1.3^{206}$ to $1.4 .^{207}$ For Gd and Sm-doped powders, values range from 1.16 to $1.46 .^{207}$ It is therefore clear that both the isothermal and isobaric Grüneisen parameters are sensitive to material factors such as grain size and defect concentration (extrinsic and intrinsic) and require further exploration in these parameter ranges.

\subsection{The effect of anisotropic strain on Raman spectra}

Ceria thin films differ from bulk samples in several aspects. The film thickness is typically much smaller than the information depth of a Raman microscope. Therefore, substrates with no overlapping Raman peaks should be chosen, and acquisition time is conventionally longer than for bulk pellet samples which have a mm-sized sample thickness. The grain size of thin films varies from tens of nanometers in polycrystalline films to very large domains in epitaxial films. This can lead to broadening and red shift of the $F_{2 g}$ peak, as observed for nanocrystalline Ceria films with grain size below $50 \mathrm{~nm} .^{208}$
Most importantly, thin films are usually strained, due to mechanical interaction with the substrate, even when they are polycrystalline. ${ }^{26,59,209}$ In epitaxial films, strain is proportional to the mismatch in substrate and film lattice parameters, but also appears in polycrystalline films, due to the deposition process. This strain, can increase or impede the ionic conductivity, e.g. shown by Harrington et al. ${ }^{210}$ or Schweiger et al. ${ }^{26}$ and decrease the reduction enthalpy. ${ }^{211}$ Therefore, knowledge and manipulation of the strain state is of high technological importance.

Raman spectroscopy was shown to be a useful non-destructive tool to assess strain in such devices, or monitor crystallization of amorphous films. ${ }^{212,213}$ For example, Shi et al. ${ }^{137}$ probed $20 \mathrm{~mol} \%$ Gd-doped ceria films, which were partly substrate-supported and partly free-standing membranes. The authors relate a $F_{2 g}$ peak shift measured by Raman spectroscopy to variations in the local strain states (compressive vs. tensile). Also Schweiger et al. modulated strain by changing the number of multi-layer interfaces in a hetero-structure consisting of alternating Gd-doped ceria and erbia layers. ${ }^{26,27}$ For larger strain levels, increased asymmetry of the unit cells was deduced from the Raman spectra and confirmed by high-resolution transmission electron microscopy (HRTEM) at the interfaces.

Even though an effect of anisotropic strain on Raman shifts of ceria films was reported by various authors, ${ }^{26,201,209,212}$ the effect of strain on vibrational lattice dynamics - and thereby Grüneisen parameters remains only partly understood. Iguchi et $a .^{207}$ suggested that since films of ceria and doped ceria experience anisotropic stress conditions, the Grüneisen relation as derived from high pressure experiments has to be modified for the biaxial modulus. This approach has also been adapted by Li et al. ${ }^{201}$ However, deviations from the relation in eqn (35) are also useful to learn about the properties of ceria materials: Kraynis et $a l^{209}$ directly measured the equivalent Grüneisen parameter for doped ceria thin films, by comparing lattice volume and $F_{2 g}$ peak position between strained thin films and relaxed fee-standing membranes. They found that the $\mathrm{F}_{2 \mathrm{~g}}$ Raman shift correlates qualitatively with the unit cell volume in agreement with eqn (35), but the equivalent Grüneisen parameter under biaxial strain is much lower than under isostatic pressure. This comparison suggested that the basic assumptions underlying the Grüneisen model (quasi-harmonicity and elasticity) are not preserved in the case of doped ceria thin films under anisotropic strain. This helped emphasize the role of defects when anisotropic stresses are applied to ceria materials, as summarized in a recent review. ${ }^{135}$

\subsection{Investigation of Ceria defect-chemistry by Raman spectroscopy: the effect of reduction and acceptor dopants}

Modification of the defect-chemistry of ceria, via either reduction of the undoped material or aliovalent doping to form solid solutions (e.g., with trivalent cations partially replacing $\mathrm{Ce}^{4+}$ ), markedly influences the Raman spectra: (i) the $\mathrm{F}_{2 \mathrm{~g}}$ mode frequency shifts in concert with changes of the lattice constant, (ii) a splitting of the $\mathrm{F}_{2 \mathrm{~g}}$ mode occurs and (iii) new modes emerge at both higher and lower wave numbers than that of the dominant $\mathrm{F}_{2 \mathrm{~g}}$ mode. These three types of changes are described 


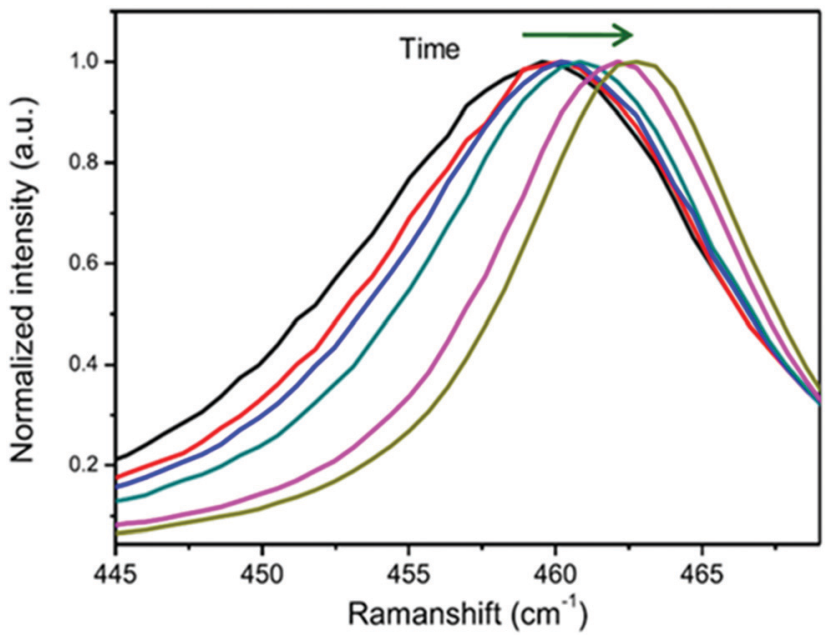

Fig. 23 Raman time series during the oxidation of an oxygen nonstoichiometric ceria film in air; the $F_{2 g}$ peak position shifts from $\sim 459 \mathrm{~cm}^{-1}$ to $465 \mathrm{~cm}^{-1}$. Spectra were normalized to $F_{2 g}$ peak height. Reproduced from ref. 201 with permission from Elsevier, copyright 2016

below in the context of chemical changes induced by reduction and doping.

4.4.1 Reduction of ceria probed by Raman spectroscopy. Reduction of ceria is accompanied by an expansion of the average lattice parameter, ${ }^{51,153}$ which in turn causes a shift of the $F_{2 g}$ mode to lower wavenumbers. Direct evidence for such behavior is presented in Fig. 23. The time series shows oxidation of a reduced $\mathrm{CeO}_{2-\delta}$ film in air as measured by Li et al. ${ }^{201}$ The peak position increases from $\sim 459 \mathrm{~cm}^{-1}$ for reduced $\mathrm{CeO}_{2-\delta}$ to $465 \mathrm{~cm}^{-1}$, which is characteristic of fully oxidized $\mathrm{CeO}_{2}$, as described in ref. 201. Because reduction of ceria induces chemical expansion, ${ }^{51}$ the trend in the peak position with oxidation state is as expected. In another experiment, Nenning et al. ${ }^{28}$ reported that a $200 \mathrm{~nm}$-thin $\mathrm{Ce}_{0.8} \mathrm{Gd}_{0.2} \mathrm{O}_{2-\delta}$ film, which was tested for memristive properties, could be reduced electrochemically at room temperature by application of a very large electric field of $8 \times 10^{7} \mathrm{~V} \mathrm{~m}^{-1}$ in across-plane direction for 90 minutes. The $4 \mathrm{~nm}$ thin Platinum top electrode enables the laser light to reach the electric field-stressed oxide and record Raman spectra. The comparison of the Raman spectra before and after the high field pulse showed a clear softening (shift to lower wave numbers) of the $\mathrm{F}_{2 \mathrm{~g}}$ mode, which is shown in Fig. 24. Analogous trends were reported by Ackermann et al. ${ }^{214}$ and by Lee et al. ${ }^{215}$ who observed redshifts of the $\mathrm{F}_{2 \mathrm{~g}}$ mode for thermally and thermochemically reduced undoped and Gd-doped ceria powders. The sensitivity of the $F_{2 g}$ peak position to temperature and oxygen nonstoichiometry presents interesting opportunities for in situ characterization of Gd-doped ceria used in a solid oxide fuel cell. ${ }^{185}$ Very recently, Sediva et al. ${ }^{216}$ demonstrated that it is possible to simultaneously acquire Raman spectra and control oxygen non-stoichiometry through electrochemical pumping of oxygen into and out of thin films deposited on an oxygen ion conductor. This approach may allow quantitative correlations between defect chemistry on the one hand and Raman shifts
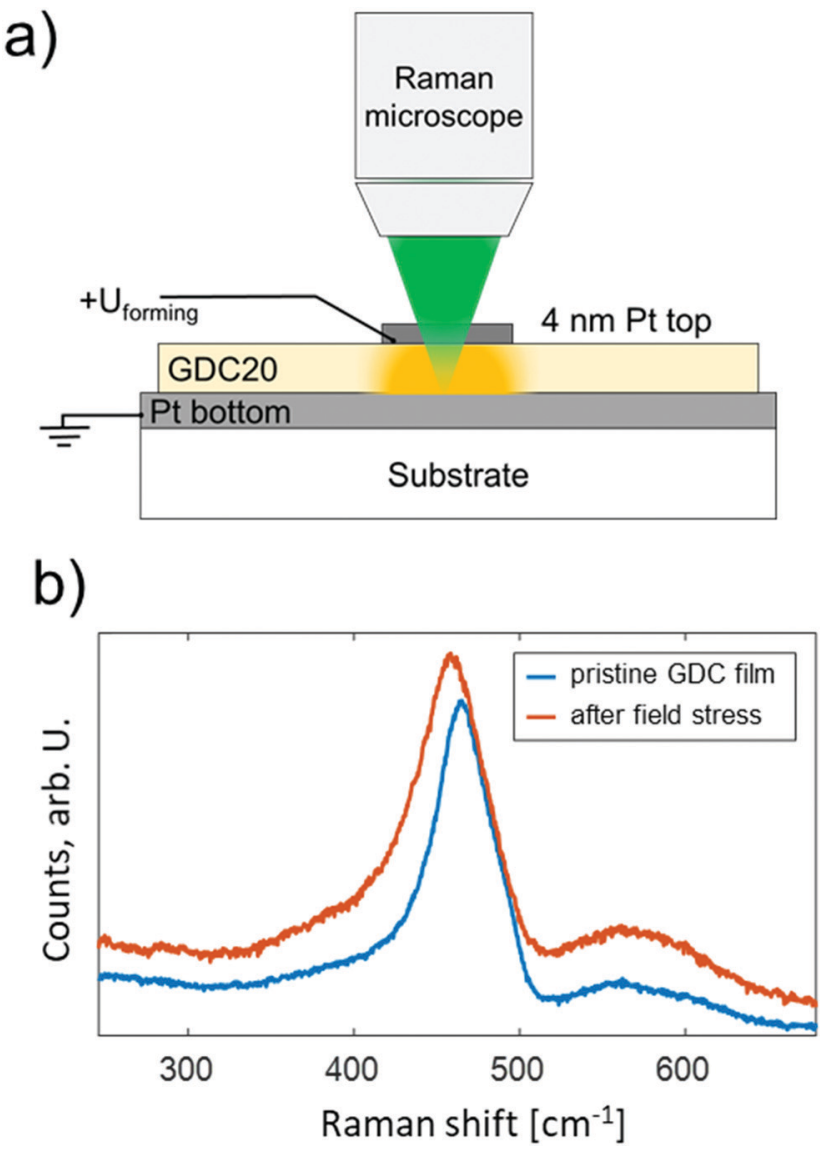

Fig. 24 An example of using Raman spectroscopy as a tool for detecting the effect of high electric fields on the defect-chemistry of ceria solid

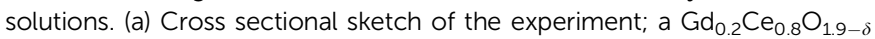
thin film is prepared between two metallic electrodes - due to the very thin top electrode (4 $\mathrm{nm} \mathrm{Pt}$ ), the Raman laser reaches the $\mathrm{Gd}_{0.2} \mathrm{Ce}_{0.8} \mathrm{O}_{1.9-\delta}$ film underneath. (b) Normalized Raman spectra before and after application of a high electric field ( $800 \mathrm{kV} \mathrm{cm}^{-1}$ for 90 minutes). Adapted from ref. 28.

and the emergence of defect-related peaks on the other hand, in ceria based solid solutions.

4.4.2 Effect of aliovalent doping on the Raman spectra of ceria solid solutions. Replacing $\mathrm{Ce}^{4+}$ ions with cations having an ionic radius larger than the critical radius for zero lattice expansion ( $\sim 102 \AA$, viz. Fig. 15) shifts the Raman frequency towards lower Raman wavenumbers, whereas the opposite is true for smaller cations. ${ }^{217}$ This trend is clearly visible in Fig. 25, where the Raman shift of the $F_{2 g}$ mode is compared to the XRD-derived lattice parameter for ceria solid solutions with dopant concentration of $20 \mathrm{~mol} \%$. To demonstrate this behavior more clearly, the Rupp research group contributed XRD and Raman results on sintered ceria pellets with $20 \mathrm{~mol} \%$ Y, Er, Gd and La (red dots) as dopants, which are complementary to the published values from Pikalova et al. for the dopants of $\mathrm{Yb}, \mathrm{Ho}, \mathrm{Dy}, \mathrm{Gd}, \mathrm{Sm}, \mathrm{La}^{124}$ (black diamonds). Experimental details are available in the ESI, $\dagger$ Section S2. Analyzing the data which stem now from independent ceramic preparation protocols, it can be concluded that a linear relationship exists between the lattice parameter and the $\mathrm{F}_{2 \mathrm{~g}}$ Raman mode 
(a)

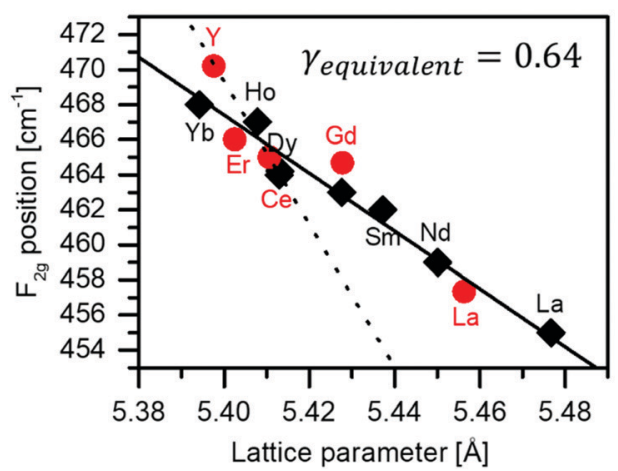

(b)

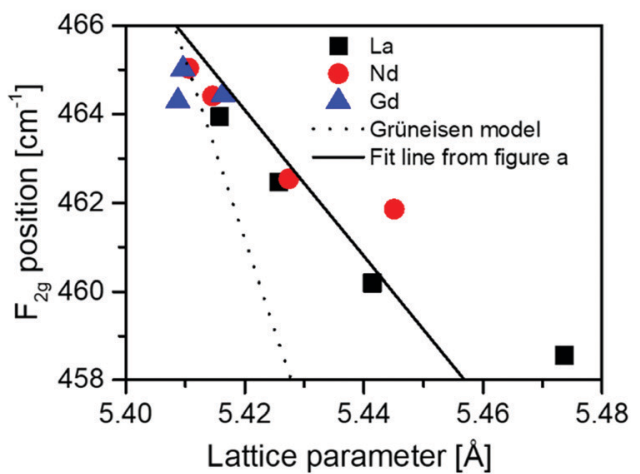

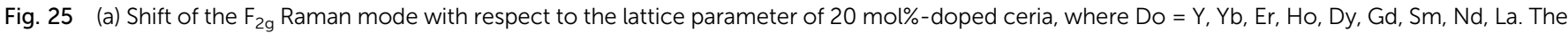

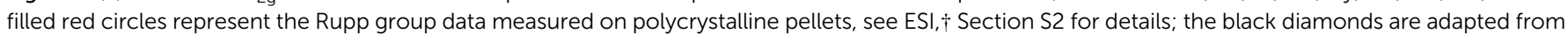

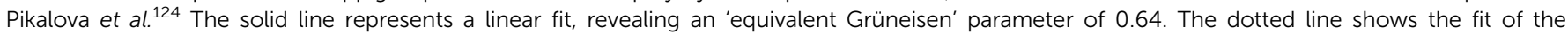

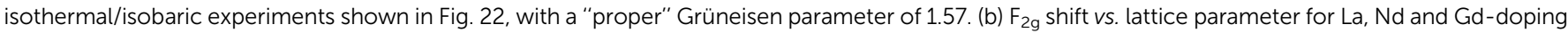

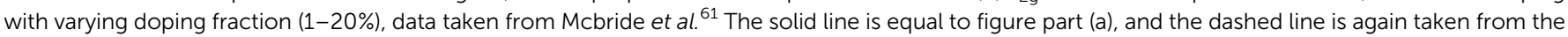
isothermal/isobaric experiment.

position, denoted by solid black line in Fig. 25(a). The fit results in a relatively lowered "equivalent Grüneisen parameter" of 0.64 , when compared to values obtained by hydrostatic compression or thermal expansion. ${ }^{200,206,207}$ The dotted line shows for comparison the fit line that was obtained for determining the isobaric/isothermal Grüneisen parameter, given in Fig. 22. Although the trend is qualitatively the same, doping produces a much weaker $F_{2 g}$ position change compared to pressure or temperature (in the range higher than $0{ }^{\circ} \mathrm{C}$ ) related changes in lattice parameter.

The Grüneisen model is applicable only if average bond lengths scale with the lattice parameter and symmetry is maintained. This is, however not the case, as shown in the EXAFS results in Fig. 12 and 15. Average Cat-O distances (which notably define bond stiffness) have no simple relation to the dopant radius or lattice parameter changes. Moreover, as shown in Fig. 14, the Ce-O distance (as measured by EXAFS) depends on the dopant species, reflecting varying degrees of local strain and suggesting variations in the degree of dopantvacancy association and extent of non-periodic deviation from the cubic fluorite symmetry. Given these findings from EXAFS, it is not surprising that the Grüneisen model is not valid. On the contrary, it is surprising that a simple linear relationship between the $F_{2 g}$ shift and lattice parameter is found at all as function of dopant radius across ten different dopant species. Noteworthy, phonon softening in Sm and Gd doped ceria was also predicted by DFT calculations by Jung et al. ${ }^{218}$ Varying the concentration of the extrinsic dopant also influences the shift of the ceria $F_{2 g}$ Raman mode. ${ }^{25,60,61,217,219}$ For example, McBride et al. measured Raman shifts and lattice parameters for $\mathrm{Gd}, \mathrm{Nd}$ and La doped ceria with $3-20 \mathrm{~mol} \%$ dopant, ${ }^{61}$ and the data are shown in Fig. 25b. Perhaps not surprisingly, the correlation in this case is not as strongly linear because of effects from both dopant concentration and dopant radius are reflected in the data. Significantly, analogous to variation of the dopant radius, changing the lattice parameter by changing the dopant content causes a much smaller $\mathrm{F}_{2 \mathrm{~g}}$ peak shift compared to isostatic pressure. Although for the dopant concentration ranges considered the material remains in the fluorite phase (according to XRD), as already discussed, doping causes local distortions and deviations from fluorite symmetry in a dopant-specific fashion, Fig. 15. Therefore, it is not particularly surprising that a single "equivalent Grüneisen parameter" is an imperfect fit of the data.

An interesting analogy can be drawn between the effects of doping and anisotropic strain effects on ceria Raman spectra. Kraynis et al. studied the Grüneisen parameter of $\mathrm{Eu}^{3+}$ and $\mathrm{Sm}^{3+}$-doped ceria thin films under biaxial strain relaxation. ${ }^{209}$ Similar to the trivalent doping effect discussed above, a value of 0.4-0.6 was found for the equivalent Grüneisen parameter. Gopal et al. showed that biaxial strain, whether compressive and tensile, increases the reducibility of $\mathrm{CeO}_{2}$ thin films. ${ }^{211}$ Thus, both trivalent doping and strain will introduce defects into the structure. Under biaxial strain, the bond stiffness no longer changes isotropically. As elaborated in Section 3.2.4 the same is also true in doped systems for local bond distances near oxygen vacancies or dopant ions, where deviations from the cubic fluorite symmetry occur. This causes some analogies in the properties of doped and anisotropically strained ceria.

4.4.3 Defect-induced splitting of the $F_{2 g}$ Raman mode. Beyond creating an average lattice expansion or contraction, extrinsic doping of ceria generates bonds that are non-equivalent. ${ }^{58} \mathrm{In}$ addition, scattering of phonons at oxygen vacancies decreases the phonon correlation length. ${ }^{208}$ These effects increase the FWHM of the $\mathrm{F}_{2 \mathrm{~g}}$ mode and create a weakly separated vibrational mode at $\sim 480 \mathrm{~cm}^{-1}$, further denoted $\Delta_{480}$ mode, which is clearly visible in Fig. 26, where the $\mathrm{F}_{2 \mathrm{~g}}$ modes of $20 \mathrm{~mol} \% \mathrm{Y}, \mathrm{Er}$, Gd and La doped ceria are plotted. In most literature reports, this feature is ignored and the $F_{2 g}$ mode for doped ceria is fitted with a single Lorentzian peak. Papers that discuss the $\Delta_{480}$ mode propose different explanations. ${ }^{167,220-222}$ Aškrabić et al. studied $\mathrm{Ce}_{0.85} \mathrm{Gd}_{0.15} \mathrm{O}_{2-\delta}$ nanocrystals and attributed the $\Delta_{480}$ mode to monoclinic 


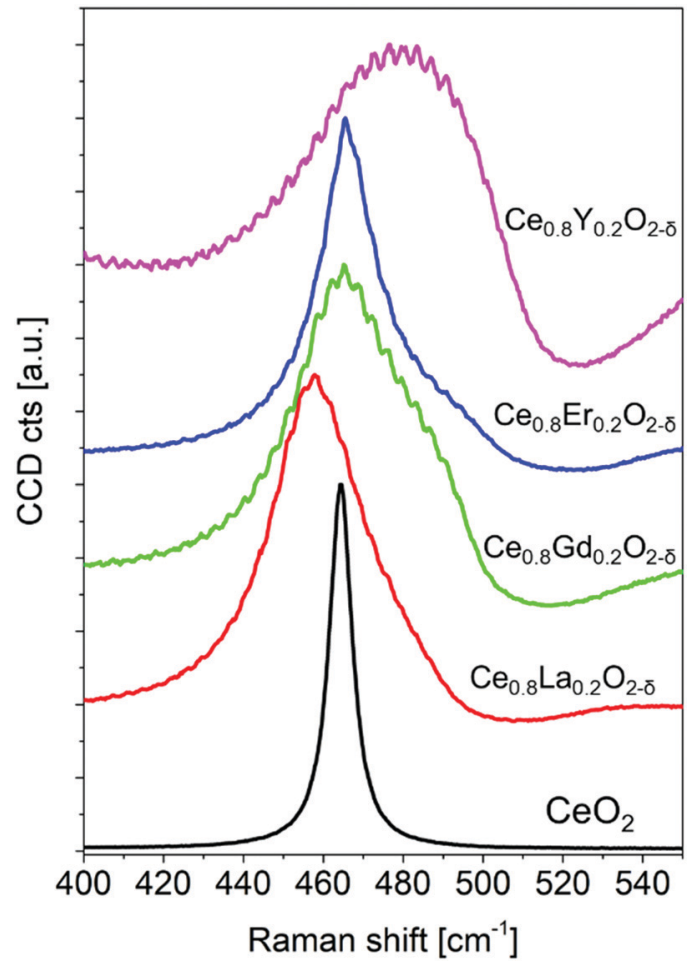

Fig. 26 Raman spectra around the $F_{2 g}$ peak in ceria pellets doped with $20 \mathrm{~mol} \% \mathrm{La}, \mathrm{Gd}$, Er, and Y, normalised to the $F_{2 g}$ peak height established by Rupp group, see ESI, $\uparrow$ Section $\mathrm{S} 2$ for experimental details.

$\mathrm{Gd}_{2} \mathrm{O}_{3},{ }^{220}$ although the strongest Raman peak of the $\mathrm{Gd}_{2} \mathrm{O}_{3}$ phase at $370 \mathrm{~cm}^{-1}$ was not observed, rendering this assignment rather unlikely. Interestingly, a $\Delta_{480}$ peak is also observed in oxygen nonstoichiometric nanocrystalline $\mathrm{CeO}_{2-\delta}{ }^{221}$ The Tyagi group discussed the appearance of the $\Delta_{480}$ mode, ${ }^{167}$ and attributed it to the oxygen vibration around the $\mathrm{Gd}^{3+}$ dopant cation in the host fluorite lattice. However, when comparing EXAFS and Raman results for different dopants, the systematic blue shift of the shoulder cannot be explained; the longer La-O bonds should be less stiff and oscillate slower than $\mathrm{Ce}-\mathrm{O}$ bonds. In contrast, the separation of $\mathrm{F}_{2 \mathrm{~g}}$ and $\Delta_{480}$ mode for differently doped ceria, as plotted in Fig. 27, is almost independent of the dopant ion radius. Thus, assignment of the $\Delta_{480}$ peak, which appears almost irrespective of the nature of the trivalent dopant, to a single type of point defect remains speculative. Furthermore, the EXAFS studies presented above indicate that upon acceptor doping, $\mathrm{Ce}-\mathrm{O}$ and Do-O bonds become non-equivalent and also exhibit significant angular deformations, further highlighting that many types of non-equivalent bonds exist in doped ceria. ${ }^{37,38,45,58,223}$ In conclusion, the $\Delta_{480}$ mode most likely originates from the nonequivalence of Cat-O bonds in doped or reduced ceria, but a unique assignment to a specific defect type remains elusive.

4.4.4 Defect-modes in Raman spectra of ceria and its solid solutions. In addition to frequency shifts and splitting of the $\mathrm{F}_{2 \mathrm{~g}}$ Raman mode, extrinsic doping or reduction of ceria leads to the appearance of additional modes with frequencies near $270 \mathrm{~cm}^{-1}$ and $550-600 \mathrm{~cm}^{-1} \cdot{ }^{224}$ The Raman spectrum of a $\mathrm{Ce}_{0.8} \mathrm{Gd}_{0.2} \mathrm{O}_{1.9}$ polycrystal is shown as an example

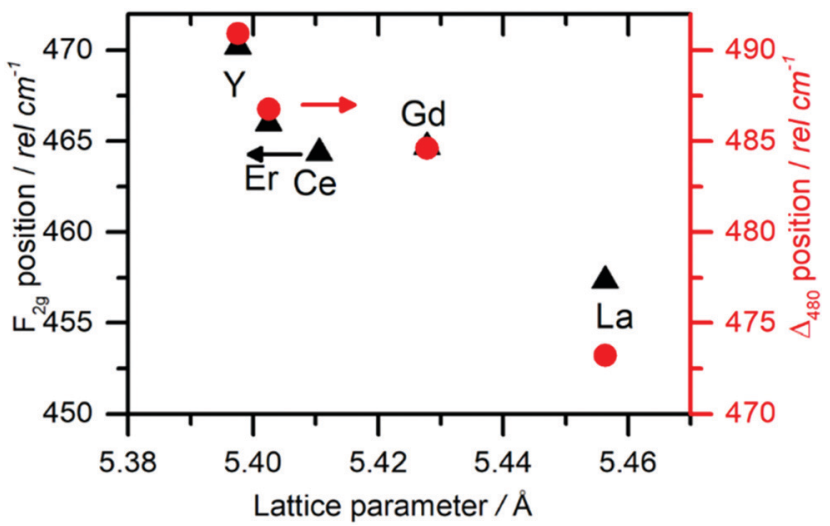

Fig. 27 Peak positions of the $F_{2 g}$ and $\Delta_{480}$ modes vs. the lattice parameter in the Raman spectra of $20 \mathrm{~mol} \%$ rare earth-doped ceria solid solutions. Raman spectra were fitted using the peak model shown in Fig. 28, and lattice parameters are given in ESI, $\dagger$ Table S14.

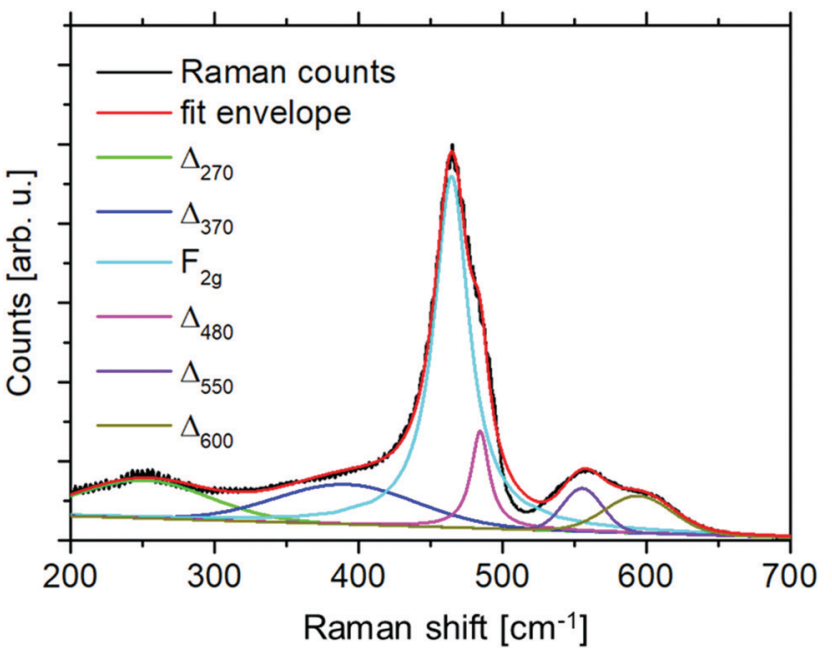

Fig. 28 Raman spectrum of a high temperature sintered $\mathrm{Ce}_{0.8} \mathrm{Gd}_{0.2} \mathrm{O}_{1.9}$ pellet, with peak fit for the $\mathrm{F}_{2 \mathrm{~g}}$ vibrational mode and various defect-related Raman peaks, labeled with $\Delta$. Experimental details are in the ESI, $\uparrow$ Section S2 measured by Rupp group on polycrystalline pellets.

in Fig. 28. See ESI, $\uparrow$ Section S2.1 for experimental details. Since most defect related Raman modes have no clearly assigned vibrational symmetry, in the following these are simply labeled by their approximate Raman shift as $\Delta_{270}, \Delta_{370}, \Delta_{480}, \Delta_{550}$, and $\Delta_{600}$. There is general agreement in the literature that these bands appear due to the presence of the dopant ions and oxygen vacancies, but their origin is a source of controversy. Authors either attribute these to oxygen vacancies, ${ }^{61,225}$ or to formation of associates between the dopant and oxygen vacancies. ${ }^{224}$

While the large peak width of the defect bands led to an initial interpretation that they occur due to second-order scattering, ${ }^{61}$ Nakajima et al. showed by careful evaluation of the temperature dependence of the peak intensities that they are first-order in nature. ${ }^{224}$ Comparison of different dopants $\left(\mathrm{La}^{3+}, \mathrm{Y}^{3+}, \mathrm{Zr}^{4+}\right),{ }^{224}$ and also $\mathrm{Hf}^{4+19}$ led to the observation that the $\Delta_{270}$ and $\Delta_{600}$ peaks are present for all dopants, ${ }^{224}$ and the $\Delta_{600}$ peak is even found in $\mathrm{CeO}_{2}$ nanocrystals. ${ }^{226,227}$ 
Although many defect related peaks appear to be rather insensitive to the type of defect, the $\Delta_{550}$ peak is only found when oxygen vacancies are present $\mathrm{t}^{24}$ and is therefore a fingerprint for oxygen vacancies.

If we compare the frequency of the defect bands with the phonon dispersion curves for ceria in Fig. 21, we see that aside from the $\mathrm{F}_{2 \mathrm{~g}}$ vibration, with phonon frequency of $465 \mathrm{~cm}^{-1}$, two additional phonon branches of $\mathrm{F}_{1 \mathrm{u}}(\mathrm{LO})$ and $\mathrm{F}_{1 \mathrm{u}}$ (TO) symmetry are relevant and have Brillouin zone center frequencies of $\sim 590$ and $\sim 290 \mathrm{~cm}^{-1}$. These phonon modes are not Raman active in the fluorite structure, which is, however, not preserved in the nearest and next nearest neighbor shells of point-defects, as elaborated in the EXAFS section. Remarkably, not only oxygen vacancies but also zirconia and hafnia dopants, that have a significant ionic radius mismatch, i.e., $\mathrm{Zr}^{4+}(0.84 \AA)$ and $\mathrm{Hf}^{4+}(0.83 \AA)$, compared to $\mathrm{Ce}^{4+}(0.97 \AA),{ }^{69}$ can be sufficient to activate the $\Delta_{600}$ and $\Delta_{270}$ modes. Although the intensity of defect-related Raman peaks depends strongly on the type of dopant (see Fig. 29a), the $\Delta_{600}$ position appears correlated with the $F_{2 g}$ peak position, Fig. 29b, which further supports the relation to the $\mathrm{F}_{1 \mathrm{u}}(\mathrm{LO})$ phonon mode.

The relative intensity of the defect-related Raman modes is significantly enhanced by using a UV laser for excitation. ${ }^{228,229}$ In the $\Delta_{550}$, and $\Delta_{600}$ defect region this effect was attributed by the authors primarily to the shallow penetration depth of UV radiation $(\lambda \approx 365 \mathrm{~nm}$ ), consequently leading to observation of the defect enriched surface at this excitation wavelength. Recently, also resonant enhancement of these defect modes was observed for undoped and a series of $\mathrm{Lu}, \mathrm{Gd}, \mathrm{Sm}$ and La doped ceria materials. ${ }^{230}$ A full set of LO mode harmonics (1-6LO) was assigned to undoped ceria. The study emphasized that under resonant conditions the $\Delta_{600}$ defect region is convoluted with the $1 \mathrm{LO}$ resonantly enhanced mode and therefore only the vacancy related $\Delta_{550}$ of the two defect bands can be resolved. The enhancement of the $\Delta_{550}$ vacancy related band under the UV excitation, enabled to observe its blue shift as function of dopant mol\% for $\mathrm{Gd}$ and $\mathrm{Sm}$ doped ceria, in contrast to the simultaneous red shift of the $F_{2 g}$ mode. This observation is in direct agreement with Fig. 15 where EXAFS derived $\mathrm{Ce}-\mathrm{O}$ and Do-O distances for Sm and Gd doped ceria contract as function of mol\%, while the lattice parameter shows expansion. The correlation of Raman and EXAFS data in this case, indicate that oxygen vacancy defects are likely in the vicinity of the contracting interatomic distances.

To continue the discussion of resonant effects, resonant enhancement in the defect region of Pr-doped ceria was demonstrated with laser wavelength of 514-532 $\mathrm{nm} .^{231}$ This enhancement was attributed to coupling of phonon states to the $\mathrm{O}^{2-} \Rightarrow \mathrm{Pr}^{4+}$ charge transfer, responsible for the absorption maximum in the visible (red/green). The intensity of the defect bands in $\mathrm{Ce}_{0.8} \mathrm{Y}_{0.2} \mathrm{O}_{1.9}$ can be explained by the small ionic radius of $\mathrm{Y}$, which facilitates association of dopants and oxygen vacancies, as predicted by DFT calculations shown in Fig. 7. Similar results are observed at higher doping concentrations in Gd-doped ceria, where the periodic arrangement of the extrinsic oxygen vacancies leads to a lowering of the crystal symmetry. ${ }^{25,167}$ (a)
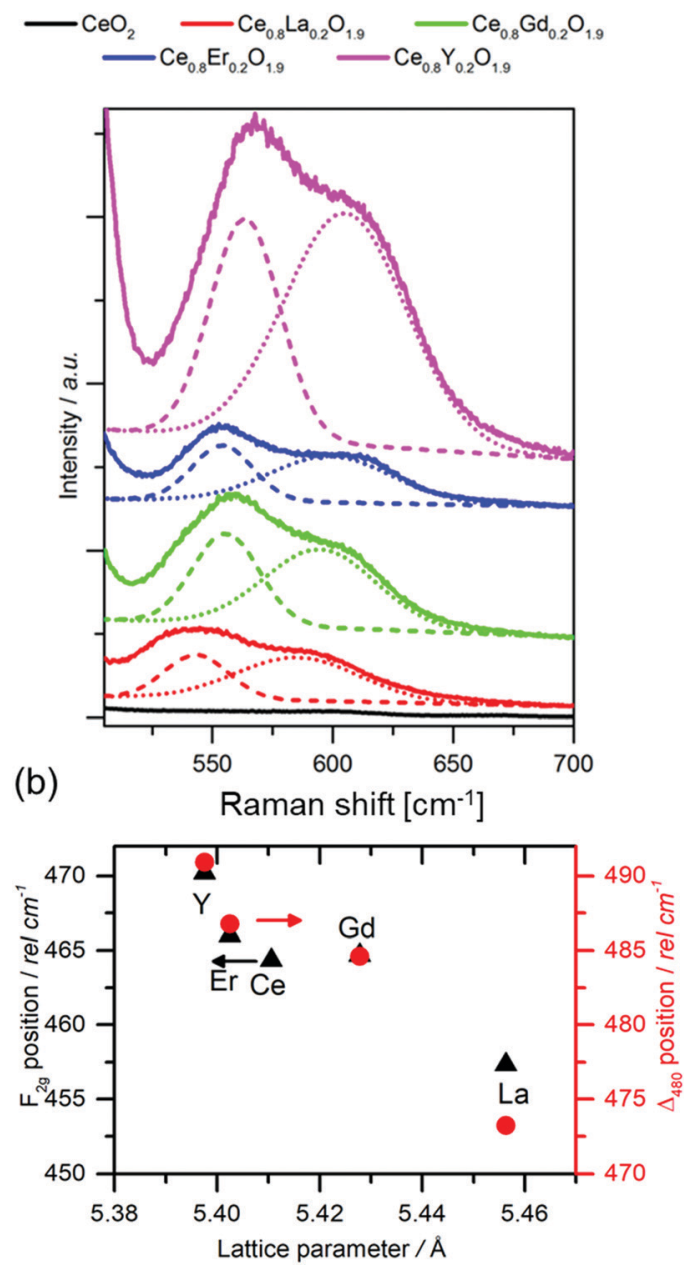

Fig. 29 (a) Magnification of the $\Delta_{550}$ and $\Delta_{600}$ Raman modes with peak fit of 20 mol\% rare-earth doped ceria with La, Gd, Er, and Y dopants. The intensity was normalized to the $F_{2 g}$ peak. (b) Fitted peak positions as a function of dopant radius. See ESI, $\uparrow$ Section S2.1 for details from pellets synthesized and measured by Rupp team.

The transition occurs at lower dopant concentrations for Yttriadoped Ceria because of the small size of the $\mathrm{Y}^{3+}$ ion, which leads to lattice instabilities relaxed through the structural transition.

4.4.5 Double fluorite ordering observed by Raman spectroscopy. The onset of double-fluorite ordering can be observed by Raman spectroscopy, even at doping levels where pure fluorite phase is detected by XRD. The fingerprint of the fluorite-double fluorite transition is the $\Delta_{370}$ peak. It was observed by variation of dopant fractions below and above double-fluorite phase formation by Schmitt et al. ${ }^{25}$ for 3-30 mol\% Gd-doping and by Artini et al. for 10-100 mol\% Sm and Gd-doping, ${ }^{60}$ as well as Ikuma et al. by Y doping. ${ }^{232}$ This peak appears at doping levels corresponding to the upper edge of the fluorite phase, and sharply increases intensity above solid solution doping limit (20-30 mol\%), when double-fluorite is detected by XRD. ${ }^{36}$ In addition, TEM measurements of $20 \mathrm{~mol} \%$ Gd-doped ceria, which is fluorite phase according to $\mathrm{XRD},{ }^{60}$ show already the appearance of nano-domains with double-fluorite like structure. ${ }^{164}$ 
For large dopant fractions, which are $>50 \mathrm{~mol} \%$, Artini et al. have presented a method for calculating the average number of neighboring oxygen ions at the dopant first coordination shell (1NN) by evaluation of the $\mathrm{F}_{2 \mathrm{~g}}$ peak position. ${ }^{233}$ The method shows that Raman spectroscopy can serve as a descriptor for local environments that are not detectable by XRD. However, the assumption of average eightfold coordination around $\mathrm{Ce}^{4+}$ is required for the calculation, a condition that is often violated in the real material. ${ }^{233}$

4.4.6 Related observations by NMR spectroscopy. Different nearest neighbor configurations or coordination numbers produce distinct shifts in NMR spectra of ${ }^{17} \mathrm{O}$ (for all ceria compositions) and ${ }^{87} \mathrm{Y}$ (for the case of yttria ceria solid solutions). From the areas of these peaks, probabilities of nearest neighbor configurations can be calculated and compared to the random distribution. Evaluation of ${ }^{17} \mathrm{O}$ spectra reveal close to random distribution of dopant ions at least for low doping to moderate doping levels for larger dopants (Gd,Sm,Nd,La) (resp. $<20 \mathrm{~mol} \%$ ). ${ }^{234,235}$ In the case of Y-doping, ${ }^{87} \mathrm{Y}$ spectra, on the other hand, show a strong preference of vacancy association with Y dopants, ${ }^{161,236}$ and strongly increased concentration of $\mathrm{Y}-\mathrm{V}_{\mathrm{O}}-\mathrm{Y}$ trimers, compared to a random distribution. Stronger tendency for vacancy-dopant association for small dopant radius was also observed in ionic conductivity measurements ${ }^{93}$ and predicted computationally, as shown in Fig. 7.

4.4.7 Raman spectroscopy of $\mathrm{CeO}_{2}$ nanoparticles. Interestingly, also $\mathrm{CeO}_{2}$ nanoparticles exhibit many features of doped or reduced ceria. For example, in ceria nanopowders with $5 \mathrm{~nm}$ grain size, the $\mathrm{F}_{2 \mathrm{~g}}$ peak shifts to lower wavenumbers by more than $10 \mathrm{~cm}^{-1}$, together with asymmetric peak broadening, compared to large grained (>100 nm) samples. ${ }^{226}$ These changes may originate from various effects: (i) phonon confinement, (ii) strain, (iii) size distribution, (iv) defects, (v) heterogeneous phonon relaxation times or correlation lengths..$^{208,237}$ In the literature, it was concluded that the larger lattice parameter of ceria nanoparticles is mostly responsible for the peak shift ${ }^{237}$ which is supported by DFT calculations by Apostolov et $a l^{238}$ The asymmetric broadening of the $\mathrm{F}_{2 \mathrm{~g}}$ peak can be modelled by taking into account a distribution of particle sizes (typically Gaussian) and phonon confinement. ${ }^{239,240}$ Due to the boundary conditions of a nanocrystal, also phonon modes with non-zero $k$-vector will have Raman intensity. Mathematically this is treated by a double integral, over the particle size distribution and a convolution of the Lorentzian intrinsic Raman line shape with a Gaussian function along the three phonon dispersion branches with $F_{2 g}$ symmetry, given by the equation ${ }^{208,220,226}$

$$
\begin{gathered}
I(\omega)=\sum_{i=1}^{3} \int_{0}^{\infty} \rho(L) \mathrm{d} L \int_{0}^{1} \exp \left(-\frac{q^{2} L^{2}}{8 \beta}\right) \\
\times \frac{1}{\left(\omega-\omega\left(q_{i}\right)+\Delta \omega(L)\right)^{2}+\frac{\Gamma_{0}^{2}}{4}} \mathrm{~d}^{3} q .
\end{gathered}
$$

Therein, $\omega$ is the angular frequency, $i$ is the number of the phonon branch (1-3) $L$ is the average particle size, $\rho(L)$ is the particle size distribution function and $q$ the phonon wavevector divided by the lattice constant. $\omega\left(q_{i}\right)$ is the frequency of the $\mathrm{F}_{2 \mathrm{~g}}$ phonon mode $i$ as function of the wave vector (see Fig. 21), $\Delta \omega(L)$ the $F_{2 g}$ red shift due to particle size and $\Gamma_{0}$ is the Raman FWHM of the single crystalline material (varying from 7-10 $\mathrm{cm}^{-1}$ ). The value of $\beta$ determines the strength of phonon confinement analytic models predict values of $\beta=1$ (Richter et al. ${ }^{240}$ ) or $\beta=2 \pi^{2}$ (Campbell et al. ${ }^{239}$ ) and other authors treat $\beta$ as a free fitting parameter, yielding values in the order of $40 .^{237}$ By combining phonon confinement and particle size distribution, several authors could explain the $F_{2 g}$ line shape of pure and doped Ceria nanocrystals. ${ }^{205,208,220,226,237,241}$ In summary, the effect of crystalline size on Raman spectra (especially the $\mathrm{F}_{2 \mathrm{~g}}$ peak) renders Raman spectroscopy as a suited tool to measure Ceria nanoparticle sizes in the range of 3-25 $\mathrm{nm}$.

\subsection{Conclusions from Raman spectroscopy}

Why Raman spectroscopy? For ceria and its solid solutions, Raman spectroscopy contributes to improved understanding of local structural changes in the nearest and next nearest neighbor shells of dopant ions and oxygen vacancies and is an excellent tool to investigate the defect chemistry of doped and undoped ceria, due to its sensitivity to non-periodic features. Several Raman peaks (around 370, 480, 550 and $600 \mathrm{~cm}^{-1}$ ) appear when point defects are introduced into ceria. By careful peak assignment, based on comparison of several literature reports and some new own measurements, one is able to gain deeper insight into the structure, local symmetry and to track tendencies towards defect ordering or association (e.g., under mechanical pressure, for high dopant concentration or small dopant cations, or also as recently shown in technical demonstration with respect to in situ variation of oxygen nonstoichiometry/defect changes in films ${ }^{216}$ ). Currently, there is still controversy in literature regarding, the origin of some of the defect and associate related Raman modes and applicability of the Grüneisen model in doped and strained ceria, even though quite good theoretical phonon and DFT models are available. To shed more light on assignments and to contribute to the discussion in the literature, we have synthesized and measured specifically doped ceria samples as standard, and tracked Raman characteristics which contribute to a more systematic study of the origin of the Raman-active defect modes.

Undoped, oxygen stoichiometric ceria only has one Raman active lattice oscillation mode at $465 \mathrm{~cm}^{-1}$, which is a symmetrical breathing mode of oxygen anions, called the $\mathrm{F}_{2 \mathrm{~g}}$ mode. According to the Grüneisen model, compressed bonds are stiffer, so the oscillation frequency, and $\mathrm{F}_{2 \mathrm{~g}}$ peak position, depends on the lattice parameter. The $\mathrm{F}_{2 \mathrm{~g}}$ position is therefore sensitive to doping, isostatic pressure, temperature and oxygen nonstoichiometry. For obtaining Grüneisen parameters, literature data and some new own measurements were re-evaluated as a function of pressure, strain, temperature or aliovalent doping up to $20 \mathrm{~mol} \%$. In all cases, a linear relation of $\mathrm{F}_{2 \mathrm{~g}}$ peak shift and lattice parameter is found, although the slopes differ significantly, depending on the origin of lattice contraction and expansion. Doping of ceria introduces non-equivalent Cat-O bonds and other lattice distorions, which were elaborated 
in the XAFS chapter. Therefore there is no simple, linear relationship between bond lengths and lattice parameter in doped ceria.

In doped, reduced or nanocrystalline ceria, or anisotropically strained thin films, membranes or multilayer film structures, additional modes appear in the Raman spectra, which are characterized as follows. An additional peak appears slightly above the $\mathrm{F}_{2 \mathrm{~g}}$ vibration, denoted as the $\Delta_{480}$ mode. It is likely because in ceria containing point defects, Cat-O bonds become non-equivalent.

Additionally, Raman peaks at $\sim 270$ and $\sim 590 \mathrm{~cm}^{-1}$ appear in ceria containing point defects, probably due to local symmetry reduction, enabling Raman activity of additional LO and TO phonon modes. This sensitivity towards local lattice distortions can provide useful insight into the concentration and association of defects.

Some defect-related peaks are related to specific structural features. A peak at $\sim 550 \mathrm{~cm}^{-1}$ appears only when oxygen vacancies are present, and is therefore a very good indicator for the latter. For dopant fractions close to or above the transition to double fluorite phase, a peak at $\sim 370 \mathrm{~cm}^{-1}$ is observed, and indicative for this transition. ${ }^{25,36,60}$ Generally, the relative intensity of the defect related Raman peaks can be greatly enhanced by using a UV laser for excitation. ${ }^{228-230}$

We emphasize by careful analysis and additional new standards published herein, that Raman spectroscopy is an excellent technique to probe temperature, strain and point defects and their changes in association and concentration for ceria materials with an easily accessible lab tool.

\section{Summary and outlook}

Undoped and doped ceria are among the most thoroughly investigated materials of modern lanthanide oxides and are currently in commercial use for a variety of applications. These investigations reveal that many properties of ceria based materials, such as elastic anomalies, strong electrostriction and defect thermodynamics, are not well described by simple models relying on the assumption that fluorite symmetry is preserved around isolated point defects. In the absence of an obvious crystallographic phase transition, standard XRD measurements are insufficient to describe properties that are likely caused by local lattice distortions and/or interacting defects that can develop in the presence of either extrinsic (dopant-induced) or intrinsic oxygen vacancies. This review has focused on experimental studies of the material's thermodynamic properties as well as its local structure and near neighbor ligand bonding patterns as measured by the complementary techniques of synchrotron X-ray absorption fine structure and Raman spectroscopy.

By combining these methods, we have presented quantitative evidence from the literature that local structure in acceptordoped $(\leq 20-30 \mathrm{~mol} \%)$, or oxygen-nonstoichiometric ceria does indeed deviate from the average fluorite symmetry. Defect thermodynamics, measured by mass changes of oxygen nonstoichiometric ceria, show that point-defects interact at quite low defect concentrations, especially in reduced, undoped ceria.
Only models that assume associates such as $\mathrm{Ce}^{3+}-\mathrm{V}_{\mathrm{O}}^{\bullet \bullet}-\mathrm{Ce}^{3+}$ trimers or more extended defect clusters can accurately describe oxygen nonstoichiometry in reduced ceria. For acceptor-doped ceria, the lattice parameter increases or decreases, depending on the ionic radius (Shannon) of the dopant, and a linear relationship of dopant radius and lattice parameter is found. ${ }^{61}$ Interestingly, the "zero expansion" dopant radius of $\sim 103 \mathrm{pm}$ is significantly larger than that of $\mathrm{Ce}^{4^{+}}(97 \mathrm{pm}){ }^{61}$ Also, if fluorite symmetry were preserved at the local level, $\mathrm{Ce}-\mathrm{O}$ and dopant-O bond lengths should simply scale with the lattice parameter. In contrast, EXAFS data show both Ce-O and dopant-O bond length contraction for ceria doped with ions that cause lattice expansion. Therefore, it is reasonable to conclude that local environments around defects strongly deviate from fluorite symmetry and that these are the source of some of its unusual mechanical properties, such as anelasticity and large electrostriction with the latter having a potential for new industrial applications. These local deviations from fluorite symmetry also mean that the lattice is anisotropically strained and presents opportunity for chemo-mechanical behavior which has not been identified by $\mathrm{XRD}$, which is only sensitive to periodic and non-element specific environments.

Also Raman spectroscopy, which is more readily accessible than synchrotron based XAFS, can probe symmetry, local order and bonding environments in ceria and its solid solutions. Structural changes caused by doping and reduction have a strong impact on Raman spectra. Defect-free, fluorite $\mathrm{CeO}_{2}$ has one Raman active phonon mode with $\mathrm{F}_{2 \mathrm{~g}}$ symmetry at $465 \mathrm{~cm}^{-1}$, reflecting the symmetric oxygen breathing vibration. A change in lattice parameter, caused by hydrostatic pressure or temperature, induces a linear response in the position of the $\mathrm{F}_{2 \mathrm{~g}}$ mode phonon frequency: compressed $\mathrm{Ce}-\mathrm{O}$ bonds are stiffer and therefore increase the $\mathrm{F}_{2 \mathrm{~g}}$ oscillation frequency. From a linear relationship of phonon frequency and unit cell volume (due to quasi-harmonic displacement of ions from their equilibrium positions) the "Grüneisen parameter" can be defined. In the case of doped ceria, an equivalent Grüneisen parameter can be determined by fitting $\mathrm{F}_{2 \mathrm{~g}}$ peak position $v$ s. lattice parameter of variously doped ceria solid solutions. This parameter is significantly lower than in the case of applied isostatic pressure or heating. This apparent discrepancy is explained by EXAFS measurements, which clearly reveal that a simple relation of lattice parameter and $\mathrm{Ce}-\mathrm{O}$ or dopant-O bond length does not exist. This is the reason why the Grüneisen model is not valid for measuring the effect of dopinginduced lattice parameter chances via Raman spectroscopy.

Emphasis is also placed on evidence from the literature that, in addition to a shift of the $F_{2 g}$ mode for ceria solid solutions with change in either dopant size or concentration, additional Raman modes appear with frequencies around 270, 370, 480, 550 and $600 \mathrm{~cm}^{-1}$. The positions of the modes around 270 and $600 \mathrm{~cm}^{-1}$ coincide with the calculated frequencies of optical phonon modes that are not Raman active in fluorite crystals. As elaborated earlier, doping locally lifts the cubic fluorite symmetry and therefore enables Raman activity of these defect related modes which include information on isolated oxygen pointdefects, and also associated defects such as Do- $-\mathrm{V}_{\mathrm{O}}-$ Do trimers. For differentiating between point-defect types, the $550 \mathrm{~cm}^{-1}$ 
mode in Raman spectra is important, as it only appears in acceptor-doped or reduced ceria, making it a descriptor for oxygen vacancies and their environment. The Raman peak at $370 \mathrm{~cm}^{-1}$ is a good indicator for double fluorite ordering, which appears at higher doping levels (typ. $>20 \mathrm{~mol} \%$ ). From combining measurements of oxygen nonstoichiometry, Ce-O bond length by EXAFS and symmetry reduction by Raman spectroscopy, we can conclude that doping of ceria causes strong lattice distortions around point-defects, which are probably the reason for the electro and chemo-mechanical properties.

Finally, although one may have considered that the defect structure and chemistry of ceria and its solid solutions might fall neatly into an ideally cubic description, this review has summarized experimental data from literature that demonstrate that in fact, doped ceria is rich with complexity. Changing perspective by moving away from structure-averaging techniques to quantitative and sensitive complementary methods, which can probe local structure and bonding environments, clearly reveals that, in the vicinity of point-defects, fluorite symmetry is indeed broken to an extent which depends on dopant identity, concentration, and local strain. We are hopeful that in this review, we have succeeded in presenting experimental findings that will assist future research in the field by contributing to tailored design of the defect-chemistry of ceria-based devices for solar-, thermo- and electro-chemistry applications and for industrial uses of giant electrostriction or memristive effects.

\section{Conflicts of interest}

There are no conflicts to declare.

\section{Acknowledgements}

This work was supported by the Swiss National Science Foundation under the project numbers 155986 (SNSF starting grant) and 138914. Further support was provided by the US National Science Foundation under award number DMR-1505103. JR thanks the Thomas Lord Foundation for support of her Thomas Lord Assistant Professorship at the Department of Materials Science and Engineering (DMSE) at the Massachusetts Institute of Technology. IL and AIF acknowledge the NSF-BSF program grant 2018717. AIF acknowledges support by NSF Grant number DMR-1911592. This work was supported in part by the Israeli Ministry of Science and Technology grant 3-12944. This research is made possible in part by the historic generosity of the Harold Perlman Family. The authors would like to thank Dr Mads Weber for fruitful discussions about Raman spectroscopy and Dr Ellen Wachtel for assistance with editing and scientific discussion.

\section{References}

1 H. Yao and Y. Y. Yao, J. Catal., 1984, 86, 254-265.

2 G. Haxel, J. Hedrick and G. Orris, Rare earth elementscritical resources for high technology, US Geological Survey, US Geological Survey, 2002.
3 S. J. Skinner and J. A. Kilner, Mater. Today, 2003, 6, 30-37.

4 B. C. Steele, Solid State Ionics, 2000, 129, 95-110.

5 J. W. Fergus, J. Power Sources, 2006, 162, 30-40.

6 J. Molenda, K. Świerczek and W. Zając, J. Power Sources, 2007, 173, 657-670.

7 K. Yashiro, T. Suzuki, A. Kaimai, H. Matsumoto, Y. Nigara, T. Kawada, J. Mizusaki and J. Sfeir, Solid State Ionics, 2004, 175, 341-344.

8 R. Heinzmann, I. Issac, J.-P. Eufinger, G. Ulbrich, M. Lerch, J. R. Janek and S. Indris, J. Phys. Chem. C, 2016, 120, 8568-8577.

9 T. Charoonsuk, N. Vittayakorn and T. Kolodiazhnyi, J. Alloys Compd., 2017, 695, 1317-1323.

10 W. C. Chueh, A. H. McDaniel, M. E. Grass, Y. Hao, N. Jabeen, Z. Liu, S. M. Haile, K. F. McCarty, H. Bluhm and F. El Gabaly, Chem. Mater., 2012, 24, 1876-1882.

11 S. C. DeCaluwe, M. E. Grass, C. Zhang, F. E. Gabaly, H. Bluhm, Z. Liu, G. S. Jackson, A. H. McDaniel, K. F. McCarty and R. L. Farrow, J. Phys. Chem. C, 2010, 114, 19853-19861.

12 A. Trovarelli, Catal. Rev., 1996, 38, 439-520.

13 T. Montini, M. Melchionna, M. Monai and P. Fornasiero, Chem. Rev., 2016, 116, 5987-6041.

14 J. Kašpar, P. Fornasiero and M. Graziani, Catal. Today, 1999, 50, 285-298.

15 W. C. Chueh, C. Falter, M. Abbott, D. Scipio, P. Furler, S. M. Haile and A. Steinfeld, Science, 2010, 330, 1797-1801.

16 J. R. Scheffe and A. Steinfeld, Mater. Today, 2014, 17, 341-348.

17 Y. Hao, C.-K. Yang and S. M. Haile, Chem. Mater., 2014, 26, 6073-6082.

18 M. Kuhn, S. Bishop, J. Rupp and H. Tuller, Acta Mater., 2013, 61, 4277-4288.

19 J. R. Scheffe, R. Jacot, G. R. Patzke and A. Steinfeld, J. Phys. Chem. C, 2013, 117, 24104-24114.

20 F. Call, M. Roeb, M. Schmucker, C. Sattler and R. Pitz-Paal, J. Phys. Chem. C, 2015, 119, 6929-6938.

21 W. C. Chueh, Y. Hao, W. Jung and S. M. Haile, Nat. Mater., 2012, 11, 155.

22 W. Jung, J. O. Dereux, W. C. Chueh, Y. Hao and S. M. Haile, Energy Environ. Sci., 2012, 5, 8682-8689.

23 V. A. Rojek-Wöckner, A. K. Opitz, M. Brandner, J. Mathé and M. Bram, J. Power Sources, 2016, 328, 65-74.

24 E. P. Murray, T. Tsai and S. Barnett, Nature, 1999, 400, 649.

25 R. Schmitt, J. Spring, R. Korobko and J. L. M. Rupp, ACS Nano, 2017, 11, 8881-8891.

26 S. Schweiger, M. Kubicek, F. Messerschmitt, C. Murer and J. L. Rupp, ACS Nano, 2014, 8, 5032-5048.

27 S. Schweiger, R. Pfenninger, W. J. Bowman, U. Aschauer and J. L. Rupp, Adv. Mater., 2017, 29, 1605049.

28 A. Nenning, R. Schmitt, T. Defferriere and J. L. M. Rupp, 2019, to be submitted.

29 A. S. Dezfuli, M. R. Ganjali, H. R. Naderi and P. Norouzi, RSC Adv., 2015, 5, 46050-46058.

30 W. Lin, Y.-w. Huang, X.-D. Zhou and Y. Ma, Int. J. Toxicol., 2006, 25, 451-457.

31 R. W. Tarnuzzer, J. Colon, S. Patil and S. Seal, Nano Lett., 2005, 5, 2573-2577. 
32 J. Koettgen, S. Grieshammer, P. Hein, B. O. Grope, M. Nakayama and M. Martin, Phys. Chem. Chem. Phys., 2018, 20, 14291-14321.

33 C. Artini, Inorg. Chem., 2018, 57, 13047-13062.

34 M. Coduri, S. Checchia, M. Longhi, D. Ceresoli and M. Scavini, Front. Chem., 2018, 6, 526.

35 C. Artini, M. Pani, M. M. Carnasciali, J. R. Plaisier and G. A. Costa, Inorg. Chem., 2016, 55, 10567-10579.

36 S. Presto, C. Artini, M. Pani, M. Carnasciali, S. Massardo and M. Viviani, Phys. Chem. Chem. Phys., 2018, 20, 28338-28345.

37 R. Korobko, A. Patlolla, A. Kossoy, E. Wachtel, H. L. Tuller, A. I. Frenkel and I. Lubomirsky, Adv. Mater., 2012, 24, 5857-5861.

38 R. Korobko, A. Lerner, Y. Li, E. Wachtel, A. I. Frenkel and I. Lubomirsky, Appl. Phys. Lett., 2015, 106, 042904.

39 M. Hadad, H. Ashraf, G. Mohanty, C. Sandu and P. Muralt, Acta Mater., 2016, 118, 1-7.

40 R. Newnham, V. Sundar, R. Yimnirun, J. Su and Q. Zhang, J. Phys. Chem. B, 1997, 101, 10141-10150.

41 R. Yimnirun, P. J. Moses, R. E. Newnham and R. J. Meyer, J. Electroceram., 2002, 8, 87-98.

42 R. Korobko, E. Wachtel and I. Lubomirsky, Sens. Actuators, A, 2013, 201, 73-78.

43 E. Mishuk, E. Makagon, E. Wachtel, S. Cohen, R. PopovitzBiro and I. Lubomirsky, Sens. Actuators, A, 2017, 264, 333-340.

44 N. Yavo, O. Yeheskel, E. Wachtel, D. Ehre, A. I. Frenkel and I. Lubomirsky, Acta Mater., 2018, 144, 411-418.

45 A. Kossoy, A. I. Frenkel, Y. Feldman, E. Wachtel, A. Milner and I. Lubomirsky, Solid State Ionics, 2010, 181, 1473-1477.

46 R. Gerhardt, W.-K. Lee and A. Nowick, J. Phys. Chem. Solids, 1987, 48, 563-569.

47 M. Takacs, J. R. Scheffe and A. Steinfeld, Phys. Chem. Chem. Phys., 2015, 17, 7813-7822.

48 B. Iwasaki and T. Katsura, Bull. Chem. Soc. Jpn., 1971, 44, 1297-1301.

49 T. Kobayashi, S. R. Wang, M. Dokiya, H. Tagawa and T. Hashimoto, Solid State Ionics, 1999, 126, 349-357.

50 R. Panlener, R. Blumenthal and J. Garnier, J. Phys. Chem. Solids, 1975, 36, 1213-1222.

51 S. Bishop, K. Duncan and E. Wachsman, Electrochim. Acta, 2009, 54, 1436-1443.

52 T. Horita, K. Yamaji, N. Sakai, M. Ishikawa, H. Yokokawa, T. Kawada and M. Dokiya, Electrochem. Solid-State Lett., 1998, 1, 4-6.

53 P. Manning, J. Sirman and J. Kilner, Solid State Ionics, 1996, 93, 125-132.

54 R. A. De Souza and M. Martin, MRS Bull., 2009, 34, 907-914.

55 E. Ruiz-Trejo, J. Sirman, Y. M. Baikov and J. Kilner, Solid State Ionics, 1998, 113, 565-569.

56 S. R. Bishop, K. L. Duncan and E. D. Wachsman, Acta Mater., 2009, 57, 3596-3605.

57 S. Kazlauskas, A. Kežionis, T. Šalkus and A. F. Orliukas, J. Mater. Sci., 2015, 50, 3246-3251.
58 O. Kraynis, J. Timoshenko, J. Huang, H. Singh, E. Wachtel, A. I. Frenkel and I. Lubomirsky, Inorg. Chem., 2019, 58, 7527-7536.

59 A. Kossoy, A. I. Frenkel, Q. Wang, E. Wachtel and I. Lubomirsky, Adv. Mater., 2010, 22, 1659-1662.

60 C. Artini, M. Pani, M. M. Carnasciali, M. T. Buscaglia, J. R. Plaisier and G. A. Costa, Inorg. Chem., 2015, 54, 4126-4137.

61 J. R. McBride, K. C. Hass, B. D. Poindexter and W. H. Weber, J. Appl. Phys., 1994, 76, 2435-2441.

62 D. Horlait, L. Claparede, N. Clavier, S. Szenknect, N. Dacheux, J. Ravaux and R. Podor, Inorg. Chem., 2011, 50, 7150-7161.

63 E. Wuilloud, B. Delley, W. D. Schneider and Y. Baer, Phys. Rev. Lett., 1984, 53, 202-205.

64 C. W. M. Castleton, J. Kullgren and K. Hermansson, J. Chem. Phys., 2007, 127, 11.

65 P. R. L. Keating, D. O. Scanlon, B. J. Morgan, N. M. Galea and G. W. Watson, J. Phys. Chem. C, 2012, 116, 2443-2452.

66 F. Kröger and H. Vink, J. Phys. Chem. Solids, 1958, 5, 208-223.

67 H. Tuller and A. Nowick, J. Phys. Chem. Solids, 1977, 38, 859-867.

68 H. L. Tuller and A. S. Nowick, J. Electrochem. Soc., 1979, 126, 209-217.

69 R. Shannon, Acta Crystallogr., Sect. A: Cryst. Phys., Diffr., Theor. Gen. Crystallogr., 1976, 32, 751-767.

70 M. Nolan, S. Grigoleit, D. C. Sayle, S. C. Parker and G. W. Watson, Surf. Sci., 2005, 576, 217-229.

71 T. Zacherle, A. Schriever, R. A. De Souza and M. Martin, Phys. Rev. B: Condens. Matter Mater. Phys., 2013, 87, 134104.

72 C. Zhang, M. E. Grass, Y. Yu, K. J. Gaskell, S. C. DeCaluwe, R. Chang, G. S. Jackson, Z. Hussain, H. Bluhm and B. W. Eichhorn, ACS Catal., 2012, 2, 2297-2304.

73 X. P. Han, N. Amrane, Z. S. Zhang and M. Benkraouda, J. Phys. Chem. C, 2016, 120, 13325-13331.

74 K. L. Duncan, Y. Wang, S. R. Bishop, F. Ebrahimi and E. D. Wachsman, J. Appl. Phys., 2007, 101, 044906.

75 C. B. Gopal and A. van de Walle, Phys. Rev. B: Condens. Matter Mater. Phys., 2012, 86, 8.

76 G. E. Murgida, V. Ferrari, M. V. Ganduglia-Pirovano and A. M. Llois, Phys. Rev. B: Condens. Matter Mater. Phys., 2014, 90, 115120.

77 S. Wang, H. Inaba, H. Tagawa, M. Dokiya and T. Hashimoto, Solid State Ionics, 1998, 107, 73-79.

78 W. Cai and W. D. Nix, Imperfections in crystalline solids, Cambridge University Press, 2016.

79 D. J. M. Bevan and J. Kordis, J. Inorg. Nucl. Chem., 1964, 26, 1509-1523.

80 B. Bulfin, L. Hoffmann, L. de Oliveira, N. Knoblauch, F. Call, M. Roeb, C. Sattler and M. Schmucker, Phys. Chem. Chem. Phys., 2016, 18, 23147-23154.

81 O. T. Sorensen, J. Solid State Chem., 1976, 18, 217-233.

82 J. Campserveux and P. Gerdanian, J. Solid State Chem., 1978, 23, 73-92.

83 S. Hull, S. T. Norberg, I. Ahmed, S. G. Eriksson, D. Marrocchelli and P. Madden, J. Solid State Chem., 2009, 182, 2815-2821. 
84 E. Kümmerle and G. Heger, J. Solid State Chem., 1999, 147, 485-500.

85 S. S. Naghavi, A. A. Emery, H. A. Hansen, F. Zhou, V. Ozolins and C. Wolverton, Nat. Commun., 2017, 8, 285.

$86 \mathrm{~S}$. Wang, H. Inaba, H. Tagawa and T. Hashimoto, J. Electrochem. Soc., 1997, 144, 4076-4080.

87 W. C. Chueh, W. Lai and S. M. Haile, Solid State Ionics, 2008, 179, 1036-1041.

88 W. Lai and S. M. Haile, J. Am. Ceram. Soc., 2005, 88, 2979-2997.

89 S. R. Wang, T. Kobayashi, M. Dokiya and T. Hashimoto, J. Electrochem. Soc., 2000, 147, 3606.

90 J. Garnier, R. Blumenthal, R. Panlener and R. Sharma, J. Phys. Chem. Solids, 1976, 37, 368-378.

91 J. Kilner, Chem. Lett., 2008, 37, 1012-1015.

92 D. Park, J. Griffith and A. Nowick, Solid State Ionics, 1981, 2, 95-105.

93 J. Faber, C. Geoffroy, A. Roux, A. Sylvestre and P. Abelard, Applied Physics A, 1989, 49, 225-232.

94 I. E. Stephens and J. A. Kilner, Solid State Ionics, 2006, 177, 669-676.

95 S. Omar, E. D. Wachsman, J. L. Jones and J. C. Nino, J. Am. Ceram. Soc., 2009, 92, 2674-2681.

96 M. Nakayama and M. Martin, Phys. Chem. Chem. Phys., 2009, 11, 3241-3249.

97 S. Kim, R. Merkle and J. Maier, Surf. Sci., 2004, 549, 196-202.

98 J. L. Rupp, A. Infortuna and L. J. Gauckler, J. Am. Ceram. Soc., 2007, 90, 1792-1797.

99 C. B. Gopal, F. E. Gabaly, A. H. McDaniel and W. C. Chueh, Adv. Mater., 2016, 28, 4692-4697.

100 X. Guo and R. Waser, Prog. Mater. Sci., 2006, 51, 151-210.

101 H. J. Avila-Paredes, K. Choi, C.-T. Chen and S. Kim, J. Mater. Chem., 2009, 19, 4837-4842.

102 A. Tschöpe, S. Kilassonia and R. Birringer, Solid State Ionics, 2004, 173, 57-61.

103 R. Jenkins, R. Manne, R. Robin and C. Senemaud, X-Ray Spectrom., 1991, 20, 149-155.

104 A. V. Soldatov, T. S. Ivanchenko, S. Dellalonga, A. Kotani, Y. Iwamoto and A. Bianconi, Phys. Rev. B: Condens. Matter Mater. Phys., 1994, 50, 5074-5080.

105 Y. R. Wang, H. Kageyama, T. Mori, H. Yoshikawa and J. Drennan, Solid State Ionics, 2006, 177, 1681-1685.

106 H. Nitani, T. Nakagawa, M. Yamanouchi, T. Osuki, M. Yuya and T. A. Yamamoto, Mater. Lett., 2004, 58, 2076-2081.

107 H. Deguchi, H. Yoshida, T. Inagaki and M. Horiuchi, Solid State Ionics, 2005, 176, 1817-1825.

108 J. A. Van Bokhoven and C. Lamberti, X-ray absorption and $X$-ray emission spectroscopy: theory and applications, John Wiley \& Sons, 2016.

109 T. Heenan, D. Brett and P. Shearing, J. Phys.: Conf. Ser., 2017, 012020.

110 D. Koningsberger and R. Prins, X-ray absorption: principles, applications, techniques of EXAFS, SEXAFS, and XANES, John Wiley and Sons, 1988.

111 G. Bunker, Introduction to XAFS: a practical guide to X-ray absorption fine structure spectroscopy, Cambridge University Press, 2010.
112 S. I. Zabinsky, J. J. Rehr, A. Ankudinov, R. C. Albers and M. J. Eller, Phys. Rev. B: Condens. Matter Mater. Phys., 1995, 52, 2995-3009.

113 P. Durham, J. Pendry and C. Hodges, Comput. Phys. Commun., 1982, 25, 193-205.

114 A. Bianconi, A. Marcelli, H. Dexpert, R. Karnatak, A. Kotani, T. Jo and J. Petiau, Phys. Rev. B: Condens. Matter Mater. Phys., 1987, 35, 806.

115 G. Gasperi, L. Amidani, F. Benedetti, F. Boscherini, P. Glatzel, S. Valeri and P. Luches, Phys. Chem. Chem. Phys., 2016, 18, 20511-20517.

116 A. Kossoy, Q. Wang, R. Korobko, V. Grover, Y. Feldman, E. Wachtel, A. K. Tyagi, A. I. Frenkel and I. Lubomirsky, Phys. Rev. B: Condens. Matter Mater. Phys., 2013, 87, 054101.

117 F. Zhang, P. Wang, J. Koberstein, S. Khalid and S.-W. Chan, Surf. Sci., 2004, 563, 74-82.

118 T.-S. Wu, Y. Zhou, R. F. Sabirianov, W.-N. Mei, Y.-L. Soo and C. L. Cheung, Chem. Commun., 2016, 52, 5003-5006.

119 H. Dexpert, R. Karnatak, J.-M. Esteva, J. Connerade, M. Gasgnier, P. Caro and L. Albert, Phys. Rev. B: Condens. Matter Mater. Phys., 1987, 36, 1750.

120 C. Paun, O. V. Safonova, J. Szlachetko, P. M. Abdala, M. Nachtegaal, J. Sa, E. Kleymenov, A. Cervellino, F. Krumeich and J. A. van Bokhoven, J. Phys. Chem. C, 2012, 116, 7312-7317.

121 D. E. Sayers, E. A. Stern and F. W. Lytle, Phys. Rev. Lett., 1971, 27, 1204-1207.

122 J. J. Rehr and R. C. Albers, Rev. Mod. Phys., 2000, 72, 621-654.

123 C. F. J. Konig, J. A. van Bokhoven, T. J. Schildhauer and M. Nachtegaal, J. Phys. Chem. C, 2012, 116, 19857-19866.

124 E. Y. Pikalova, A. A. Murashkina, V. I. Maragou, A. K. Demin, V. N. Strekalovsky and P. E. Tsiakaras, Int. J. Hydrogen Energy, 2011, 36, 6175-6183.

125 B. K. Teo, EXAFS: basic principles and data analysis, Springer Science \& Business Media, 2012.

126 F. W. Lytle, D. E. Sayers and E. A. Stern, Phys. Rev. B: Solid State, 1975, 11, 4825-4835.

127 E. A. Stern, D. E. Sayers and F. W. Lytle, Phys. Rev. B: Solid State, 1975, 11, 4836-4846.

128 F. De Groot and A. Kotani, Core level spectroscopy of solids, CRC press, 2008.

129 E. Stern and S. Heald, Handbook on synchrotron radiation, 1983, vol. 1, pp. 955-1014.

130 H. Wende, Rep. Prog. Phys., 2004, 67, 2105.

131 E. D. Crozier, Nucl. Instrum. Methods Phys. Res., Sect. B, 1997, 133, 134-144.

132 A. I. Frenkel and J. J. Rehr, Phys. Rev. B: Condens. Matter Mater. Phys., 1993, 48, 585-588.

133 G. Dalba, P. Fornasini, M. Grazioli, R. Gotter and F. Rocca, Physica B, 1995, 208, 135-136.

134 M. Mogensen, N. M. Sammes and G. A. Tompsett, Solid State Ionics, 2000, 129, 63-94.

135 E. Wachtel, A. I. Frenkel and I. Lubomirsky, Adv. Mater., 2018, 30, 1707455. 
136 F. Giannici, G. Gregori, C. Aliotta, A. Longo, J. Maier and A. Martorana, Chem. Mater., 2014, 26, 5994-6006.

137 Y. Shi, A. H. Bork, S. Schweiger and J. L. M. Rupp, Nat. Mater., 2015, 14, 721-727.

138 A. Kossoy, E. Wachtel and I. Lubomirsky, J. Electroceram., 2014, 32, 47-50.

139 R. T. Shannon and C. T. Prewitt, Acta Crystallogr., Sect. B: Struct. Crystallogr. Cryst. Chem., 1969, 25, 925-946.

140 S. Yamazaki, T. Matsui, T. Ohashi and Y. Arita, Solid State Ionics, 2000, 136, 913-920.

141 T. Ohashi, S. Yamazaki, T. Tokunaga, Y. Arita, T. Matsui, T. Harami and K. Kobayashi, Solid State Ionics, 1998, 113, 559-564.

142 J. Koettgen and M. Martin, J. Phys. Chem. C, 2019, 123, 6333-6339.

143 C. Artini, G. A. Costa, M. Pani, A. Lausi and J. Plaisier, J. Solid State Chem., 2012, 190, 24-28.

144 M. Scavini, M. Coduri, M. Allieta, M. Brunelli and C. Ferrero, Chem. Mater., 2012, 24, 1338-1345.

145 M. Coduri, M. Scavini, M. Allieta, M. Brunelli and C. Ferrero, Chem. Mater., 2013, 25, 4278-4289.

146 M. Coduri, P. Masala, M. Allieta, I. Peral, M. Brunelli, C. A. Biffi and M. Scavini, Inorg. Chem., 2017, 57, 879-891.

147 Y. Rosenberg, V. S. Machavariani, A. Voronel, S. Garber, A. Rubshtein, A. Frenkel and E. Stern, J. Phys.: Condens. Matter, 2000, 12, 8081.

148 A. I. Frenkel, E. A. Stern, A. Voronel and S. M. Heald, Solid State Commun., 1996, 99, 67-71.

149 A. Frenkel, A. Voronel, A. Katzir, M. Newville and E. Stern, Phys. B, 1995, 208, 334.

150 A. Frenkel, E. A. Stern, A. Voronel, M. Qian and M. Newville, Phys. Rev. B: Condens. Matter Mater. Phys., 1994, 49, 11662.

151 A. Frenkel, V. S. Machavariani, A. Rubshtein, Y. Rosenberg, A. Voronel and E. Stern, Phys. Rev. B: Condens. Matter Mater. Phys., 2000, 62, 9364.

152 P. Li, I. W. Chen, J. E. Pennerhahn and T. Y. Tien, J. Am. Ceram. Soc., 1991, 74, 958-967.

153 D. Marrocchelli, S. R. Bishop, H. L. Tuller and B. Yildiz, Adv. Funct. Mater., 2012, 22, 1958-1965.

154 H. Inaba, R. Sagawa, H. Hayashi and K. Kawamura, Solid State Ionics, 1999, 122, 95-103.

155 S. C. Shirbhate, K. Singh, S. A. Acharya and A. K. Yadav, Ionics, 2017, 23, 1049-1057.

156 S. C. Shirbhate, A. K. Yadav and S. A. Acharya, Appl. Phys. Lett., 2016, 108, 143501.

157 S. J. Hong and A. V. Virkar, J. Am. Ceram. Soc., 1995, 78, 433-439.

158 D. J. Kim, J. Am. Ceram. Soc., 1989, 72, 1415-1421.

159 N. Yavo, D. Noiman, E. Wachtel, S. Kim, Y. Feldman, I. Lubomirsky and O. Yeheskel, Scr. Mater., 2016, 123, 86-89.

160 M. Varenik, X.-D. Zhang, G. Leitus, N. Yavo, R. Carmieli, E. Wachtel, X. Guo and I. Lubomirsky, Phys. Chem. Chem. Phys., 2018, 20, 27019-27024.

161 S. Grieshammer, B. O. Grope, J. Koettgen and M. Martin, Phys. Chem. Chem. Phys., 2014, 16, 9974-9986.
162 D. A. Andersson, S. I. Simak, N. V. Skorodumova, I. A. Abrikosov and B. Johansson, Proc. Natl. Acad. Sci. U. S. A., 2006, 103, 3518-3521.

163 J. Koettgen, T. Zacherle, S. Grieshammer and M. Martin, Phys. Chem. Chem. Phys., 2017, 19, 9957-9973.

164 F. Ye, T. Mori, D. R. Ou, J. Zou, G. Auchterlonie and J. Drennan, Solid State Ionics, 2008, 179, 827-831.

165 P. A. Žguns, A. V. Ruban and N. V. Skorodumova, Phys. Chem. Chem. Phys., 2018, 20, 11805-11818.

166 P. A. Žguns, A. V. Ruban and N. V. Skorodumova, Scr. Mater., 2019, 158, 126-130.

167 A. Banerji, V. Grover, V. Sathe, S. Deb and A. Tyagi, Solid State Commun., 2009, 149, 1689-1692.

168 V. Shelukhin, I. Zon, E. Wachtel, Y. Feldman and I. Lubomirsky, Solid State Ionics, 2012, 211, 12-19.

169 Y. Li, O. Kraynis, J. Kas, T.-C. Weng, D. Sokaras, R. Zacharowicz, I. Lubomirsky and A. I. Frenkel, AIP Adv., 2016, 6, 055320.

170 K. O. Kvashnina, S. M. Butorin and P. Glatzel, J. Anal. At. Spectrom., 2011, 26, 1265-1272.

171 A. H. Clark, H. R. Marchbank, T. I. Hyde, H. Y. Playford, M. G. Tucker and G. Sankar, Phys. Scr., 2017, 92, 034002.

172 J. Timoshenko, A. Kuzmin and J. Purans, J. Phys.: Condens. Matter, 2014, 26, 055401.

173 J. Wicks and R. McGreevy, J. Non-Cryst. Solids, 1995, 192, 23-27.

174 C. I. Hiley, H. Y. Playford, J. M. Fisher, N. C. Felix, D. Thompsett, R. J. Kashtiban and R. I. Walton, J. Am. Chem. Soc., 2018, 140, 1588-1591.

175 A. Frenkel, E. A. Stern, A. Voronel, M. Qian and M. Newville, Phys. Rev. Lett., 1993, 71, 3485.

176 A. I. Frenkel, D. M. Pease, J. I. Budnick, P. Metcalf, E. A. Stern, P. Shanthakumar and T. Huang, Phys. Rev. Lett., 2006, 97, 195502.

177 H. Singh, D. Donetsky, J. Liu, K. Attenkofer, B. Cheng, J. Trelewicz, I. Lubomirsky, E. Stavitski and A. I. Frenkel, Rev. Sci. Instrum., 2018, 89, 045111.

178 T. R. Gilson and P. J. Hendra, Laser Raman Spectroscopy: A Survey of Interest Primarily to Chemists, and Containing a Comprehensive Discussion of Experiments on Crystals, Wiley-Interscience, London, 1970.

179 P. L. Stiles, J. A. Dieringer, N. C. Shah and R. P. Van Duyne, Annu. Rev. Anal. Chem., 2008, 1, 601-626.

180 R. M. Stöckle, Y. D. Suh, V. Deckert and R. Zenobi, Chem. Phys. Lett., 2000, 318, 131-136.

181 E. Bailo and V. Deckert, Chem. Soc. Rev., 2008, 37, 921-930.

182 E. Ploetz, S. Laimgruber, S. Berner, W. Zinth and P. Gilch, Appl. Phys. B: Lasers Opt., 2007, 87, 389-393.

183 P. Kukura, D. W. McCamant and R. A. Mathies, Annu. Rev. Phys. Chem., 2007, 58, 461-488.

184 P. Larkin, Infrared and Raman Spectroscopy, Elsevier, Oxford, 2011, pp. 7-25.

185 R. Maher, L. Cohen, P. Lohsoontorn, D. Brett and N. Brandon, J. Phys. Chem. A, 2008, 112, 1497-1501.

186 R. S. Drago, Physical methods in chemistry, 1977. 
187 J. Joo, T. Yu, Y. W. Kim, H. M. Park, F. Wu, J. Z. Zhang and T. Hyeon, J. Am. Chem. Soc., 2003, 125, 6553-6557.

188 J. Cui and G. A. Hope, J. Spectrosc., 2015, 940172.

189 C. Michael, H. Yuen, V. Sabnis, T. Johnson, R. Sewell, R. Smith, A. Jamora, A. Clark, S. Semans and P. Atanackovic, Opt. Express, 2008, 16, 19649-19666.

190 D. Avram, C. Rotaru, B. Cojocaru, M. Sanchez-Dominiguez, M. Florea and C. Tiseanu, J. Mater. Sci., 2014, 49, 2117-2126.

191 M. Tasumi, A. Sakamoto, T. Hieda and H. Torii, Handbook of Vibrational Spectroscopy, John Wiley \& Sons, Ltd, 2006.

192 M. I. Aroyo, A. Kirov, C. Capillas, J. M. Perez-Mato and H. Wondratschek, Acta Crystallogr., Sect. A: Found. Crystallogr., 2006, 62, 115-128.

193 S. F. Parker, Handbook of Vibrational Spectroscopy, John Wiley \& Sons, Ltd, 2006.

194 B. Eberhard, Rep. Prog. Phys., 2000, 63, 171.

195 M. A. Chesters, Handbook of Vibrational Spectroscopy, John Wiley \& Sons, Ltd, 2006.

196 H. Matsuura and H. Yoshida, Handbook of Vibrational Spectroscopy, John Wiley \& Sons, Ltd, 2006.

197 J. Buckeridge, D. O. Scanlon, A. Walsh, C. R. A. Catlow and A. A. Sokol, Phys. Rev. B: Condens. Matter Mater. Phys., 2013, 87, 214304.

198 W. H. Weber, K. C. Hass and J. R. McBride, Phys. Rev. B: Condens. Matter Mater. Phys., 1993, 48, 178-185.

199 G. Lucazeau, J. Raman Spectrosc., 2003, 34, 478-496.

200 G. A. Kourouklis, A. Jayaraman and G. P. Espinosa, Phys. Rev. B: Condens. Matter Mater. Phys., 1988, 37, 4250-4253.

201 H. Li, P. Zhang, G. Li, J. Lu, Q. Wu and Y. Gu, J. Alloys Compd., 2016, 682, 132-137.

202 U. Benedict, J. Alloys Compd., 1995, 223, 216-225.

203 S. Stecura and W. J. Campbell, Thermal expansion and phase inversion of rare-earth oxides, Bureau of Mines. College Park Metallurgy Research Center, Md., 1960.

204 S. D. Pandey, J. Singh, K. Samanta, N. D. Sharma and A. Bandyopadhyay, J. Nanomater., 2015, 16, 154.

205 Z. Popović, Z. Dohčević-Mitrović, A. Cros and A. Cantarero, J. Phys.: Condens. Matter, 2007, 19, 496209.

206 S. Dogra, J. Singh, N. D. Sharma, K. Samanta, H. Poswal, S. Sharma and A. Bandyopadhyay, Vib. Spectrosc., 2014, 70, 193-199.

207 F. Iguchi, S. Onodera, N. Sata and H. Yugami, Solid State Ionics, 2012, 225, 99-103.

208 I. Kosacki, T. Suzuki, H. U. Anderson and P. Colomban, Solid State Ionics, 2002, 149, 99-105.

209 O. Kraynis, E. Makagon, E. Mishuk, M. Hartstein, E. Wachtel, I. Lubomirsky and T. Livneh, Adv. Funct. Mater., 2019, 29, 1804433.

210 G. F. Harrington, L. Sun, B. Yildiz, K. Sasaki, N. H. Perry and H. L. Tuller, Acta Mater., 2019, 166, 447-458.

211 C. B. Gopal, M. García-Melchor, S. C. Lee, Y. Shi, A. Shavorskiy, M. Monti, Z. Guan, R. Sinclair, H. Bluhm and A. Vojvodic, Nat. Commun., 2017, 8, 15360.
212 J. L. Rupp, B. Scherrer and L. J. Gauckler, Phys. Chem. Chem. Phys., 2010, 12, 11114-11124.

213 R. Pfenninger, M. Struzik, I. Garbayo, E. Stilp and J. L. Rupp, Nat. Energy, 2019, 4, 475-483.

214 S. Ackermann, L. Sauvin, R. Castiglioni, J. L. M. Rupp, J. R. Scheffe and A. Steinfeld, J. Phys. Chem. C, 2015, 119, 16452-16461.

215 K. Lee and J. R. Scheffe, J. Sol. Energy Eng., 2019, 141, 021013. 216 E. Sediva, T. Defferriere, N. H. Perry, H. L. Tuller and J. L. Rupp, Adv. Mater., 2019, 1902493.

217 J. L. M. Rupp, E. Fabbri, D. Marrocchelli, J.-W. Han, D. Chen, E. Traversa, H. L. Tuller and B. Yildiz, Adv. Funct. Mater., 2014, 24, 1562-1574.

218 D.-H. Jung, J.-H. Lee, M. E. Kilic and A. Soon, Phys. Chem. Chem. Phys., 2018, 20, 10048-10059.

219 N. Yang, Y. Shi, S. Schweiger, E. Strelcov, A. Belianinov, V. Foglietti, P. Orgiani, G. Balestrino, S. V. Kalinin, J. L. M. Rupp and C. Aruta, ACS Appl. Mater. Interfaces, 2016, 8, 14613-14621.

220 S. Aškrabić, Z. D. Dohčević-Mitrović, M. Radović, M. Šćepanović and Z. V. Popović, J. Raman Spectrosc., 2009, 40, 650-655.

221 S. Aškrabić, Z. Dohčević-Mitrović, A. Kremenović, N. Lazarević, V. Kahlenberg and Z. V. Popović, J. Raman Spectrosc., 2012, 43, 76-81.

222 V. Grover, A. Banerji, P. Sengupta and A. K. Tyagi, J. Solid State Chem., 2008, 181, 1930-1935.

223 R. Korobko, S. K. Kim, S. Kim, S. R. Cohen, E. Wachtel and I. Lubomirsky, Adv. Funct. Mater., 2013, 23, 6076-6081.

224 A. Nakajima, A. Yoshihara and M. Ishigame, Phys. Rev. B: Condens. Matter Mater. Phys., 1994, 50, 13297-13307.

225 T. Tsuchiya, S. Miyoshi, Y. Yamashita, H. Yoshikawa, K. Terabe, K. Kobayashi and S. Yamaguchi, Sci. Technol. Adv. Mater., 2013, 14, 045001.

226 Z. D. Dohčević-Mitrović, M. J. Šćepanović, M. U. Grujić-Brojčin, Z. V. Popović, S. B. Bošković, B. M. Matović, M. V. Zinkevich and F. Aldinger, Solid State Commun., 2006, 137, 387-390.

227 Z. Wu, M. Li, J. Howe, H. M. Meyer and S. H. Overbury, Langmuir, 2010, 26, 16595-16606.

228 T. Taniguchi, T. Watanabe, N. Sugiyama, A. K. Subramani, H. Wagata, N. Matsushita and M. Yoshimura, J. Phys. Chem. C, 2009, 113, 19789-19793.

229 M. Guo, J. Lu, Y. Wu, Y. Wang and M. Luo, Langmuir, 2011, 27, 3872-3877.

230 O. Kraynis, I. Lubomirsky and T. Livneh, J. Phys. Chem. C, 2019, 123, 24111-24117.

231 A. Westermann, C. Geantet, P. Vernoux and S. Loridant, J. Raman Spectrosc., 2016, 47, 1276-1279.

232 Y. Ikuma, S. Nagasawa, N. Hayashi and M. Kamiya, J. Jpn. Soc. Powder Powder Metall., 2005, 52, 599-602.

233 C. Artini, M. M. Carnasciali, J. R. Plaisier, G. A. Costa and M. Pani, Solid State Ionics, 2017, 311, 90-97.

234 M. Ando, I. Oikawa, S. Ohki, M. Tansho, T. Shimizu, H. Maekawa and H. Kiyono, Chem. Lett., 2012, 42, 57-59.

235 I. Heinmaa, T. Joon, H. Kooskora, J. Pahapill and J. Subbi, Solid State Ionics, 2010, 181, 1309-1315. 
236 N. Kim and J. F. Stebbins, Chem. Mater., 2007, 19, 5742-5747. 237 J. E. Spanier, R. D. Robinson, F. Zhang, S.-W. Chan and I. P. Herman, Phys. Rev. B: Condens. Matter Mater. Phys., 2001, 64, 245407.

238 A. Apostolov, I. Apostolova and J. Wesselinowa, Solid State Commun., 2018, 279, 17-21.
239 I. Campbell and P. M. Fauchet, Solid State Commun., 1986, 58, 739-741.

240 H. Richter, Z. Wang and L. Ley, Solid State Commun., 1981, 39, 625-629.

241 Z. Popović, Z. Dohčević-Mitrović, M. Šćepanović, M. GrujićBrojčin and S. Aškrabić, Ann. Phys., 2011, 523, 62-74. 